University of Louisville

ThinkIR: The University of Louisville's Institutional Repository

$5-2011$

\title{
Survey of pediatric residents regarding communication disorders and augmentative and alternative communication.
}

Karen J. Coulter

University of Louisville

Follow this and additional works at: https://ir.library.louisville.edu/etd

\section{Recommended Citation}

Coulter, Karen J., "Survey of pediatric residents regarding communication disorders and augmentative and alternative communication." (2011). Electronic Theses and Dissertations. Paper 280.

https://doi.org/10.18297/etd/280

This Doctoral Dissertation is brought to you for free and open access by ThinkIR: The University of Louisville's Institutional Repository. It has been accepted for inclusion in Electronic Theses and Dissertations by an authorized administrator of ThinkIR: The University of Louisville's Institutional Repository. This title appears here courtesy of the author, who has retained all other copyrights. For more information, please contact thinkir@louisville.edu. 


\title{
SURVEY OF PEDIATRIC RESIDENTS REGARDING COMMUNICATION DISORDERS AND AUGMENTATIVE AND ALTERNATIVE COMMUNICATION \\ by \\ Karen J. Coulter \\ B.S., Illinois State University, 1992 \\ M.S., Indiana State University, 1998
}

\author{
A Dissertation \\ Submitted to the Faculty of the \\ Graduate School of the University of Louisville \\ in Partial Fulfillment of the Requirements \\ for the Degree \\ Doctor of Philosophy
}

Department of Education

University of Louisville

Louisville, Kentucky

May, 2011 
Copyright 2011 by Karen J. Coulter

All rights reserved 



\section{SURVEY OF PEDIATRIC RESIDENTS \\ REGARDING COMMUNICATION DISORDERS AND \\ AUGMENTATIVE AND ALTERNATIVE COMMUNICATION}

By

Karen J. Coulter

B.S., Illinois State University, 1992

M.S., Indiana State University, 1998

A Dissertation Approved on

March 31, 2011

by the following Dissertation Committee:

Dissertation Co-Director (Debra K. Bauder)

Dissertation Co-Director (Thomas J. Simmons)

M. Gay Masters

Joseph Petrosko

P. Gail Williams 


\section{DEDICATION}

"Two are better than one, because they have a good return for their labor: if either of them falls down, one can help the other up. But pity anyone who falls and has no one to help them up. Also, if two lie down together, they will keep warm. But how can one keep warm alone? Though one may be overpowered, two can defend themselves. A cord of three strands is not quickly broken." Ecclesiastes 4:9-12

This dissertation is dedicated to my loved ones. It is for Bryce who has patiently supported my educational process for many years. Your confidence in me and my decision sustained my perseverance. You stuck with me, provided for me and shared my sacrifices in so many ways.

This is also for my parents who believe in me and cheer me on. Your examples in character, faith, and blessing have given me a high standard I hope to one day obtain.

To all those that have poured their love into me, this achievement is yours. I will always hold each of you in my heart as I continue to 'press on' toward that to which l've been called. 


\section{ACKNOWLEDGEMENTS}

I am a turtle on a fence post. I know I didn't get there by myself, and I freely share this truth with all who will listen. To those that have helped me along the way, I want to give a sincere thank-you.

My first thanks goes to my dissertation committee, Deb, Tom, Joe, Gail and Gay. I appreciate not you're your support, but also your significant input within this research endeavor. I hope to build on what you have given to me and make you glad for your time and investment. Your many instructions won't go to waste.

I also need to recognize the individuals on my expert panel, Joseph Hersh, Alex Asamoah, Yasmin Senturias, Terry Erwin, Gordan Gowans, Kara Goodin, and Richard McChane. I know resident education is important to each of you. I couldn't have completed my research without your time and constructive input into the survey development. I appreciate all of your support and encouragement. 


\section{ABSTRACT \\ SURVEY OF PEDIATRIC RESIDENTS REGARDING COMMUNICATION DISORDERS AND \\ AUGMENTATIVE AND ALTERNATIVE COMMUNICATION}

Karen J. Coulter

March 31, 2011

Children with general communication impairments as well as complex communication needs rely on pediatricians to prescribe the services of speech language pathologists. In light of this continuing and increasing need, it is important to ascertain whether medical residents are receiving the necessary training in their educational program to fulfill their role. We need to understand how pediatric residents perceive their current level of abilities particularly within the framework of the current ACGME competencies.

The purpose of this dissertation was to identify possible differences across pediatric resident levels regarding competence within three constructs, Medical Education, Medical Knowledge and Professional Practice, with a specific focus on communication disorders and augmentative and alternative communication (AAC). A MANOVA was used to address the first three of five research questions. Upon analysis of the MANOVA, the main effect was significant for differences among the three groups of residents in their average levels of self- 
reported competence in the three constructs. Further paired comparisons found differences across pediatric levels for Medical Education and Medical Knowledge.

The fourth research question investigated the effects of demographic variables on residents' perceived competency across the three constructs. These demographic variables included gender, rotation completion, pediatric specialization educational methods, and educational time. Independent $t$ - tests were completed with Bonferroni adjustment as well as correlation coefficients. Significant findings within these variables provide further understanding of current and future pediatric resident training.

The final research question investigated the perspectives of pediatric residents regarding communication disorders and $A A C$ as part of their training. This question provided qualitative data gathered through responses to several open-ended questions. Residents were confident in their ability to identify a communication disorder or a need for $A A C$ and make referrals. At the same time, residents expressed concerns regarding educational training and appeared to not grasp the entirety of their roles.

The results of this study provide evidence for some improvements within residents' perceived competence for referrals and knowledge base. Yet, it appears that improvements are still needed regarding residents' educational opportunities, and understanding of their role within the provision of services for children with communication disorders and needing AAC. Follow-up of this 
current investigation by educational leaders and continued research within this field will support this effort. 
TABLE OF CONTENTS

PAGE

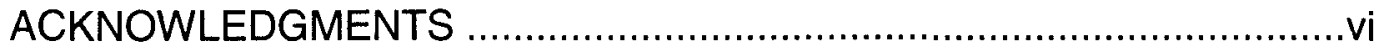

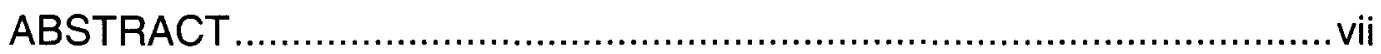

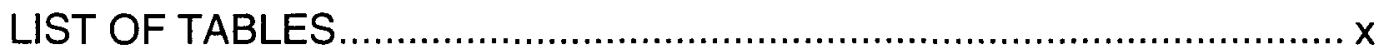

LIST OF FIGURES .........................................................................

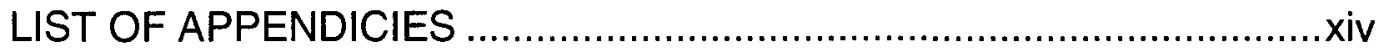

\section{CHAPTER}

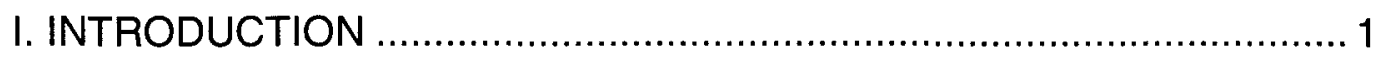

Communication Disorders .......................................................... 2

Augmentative and Alternative Communication............................... 4

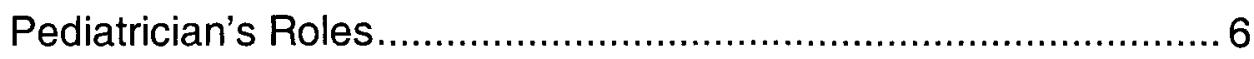

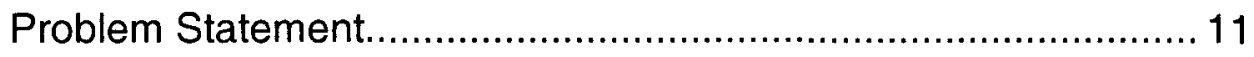

Purpose of the Study ............................................................... 13

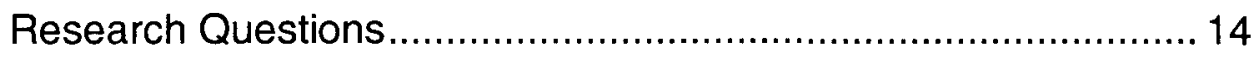

Significance of the Study.......................................................... 14

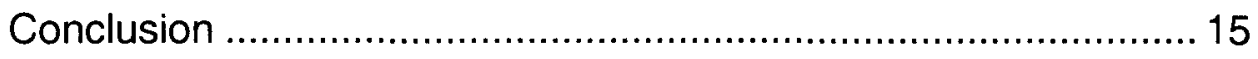

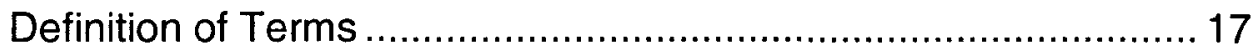

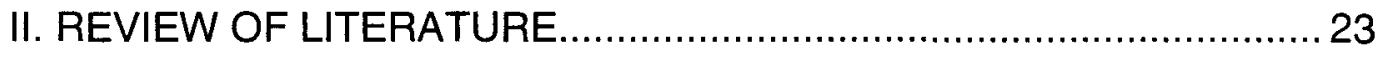

Historical Chronology of Communication Disorders ...................... 23

Historical Chronology of Augmentative and Alternative

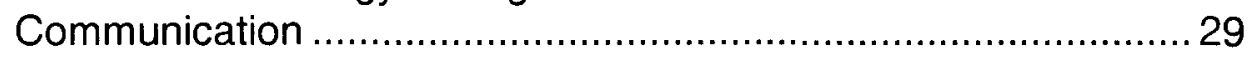


Graduate Medical Training .......................................................... 51

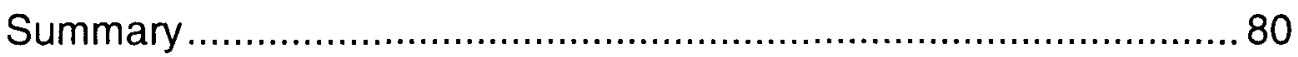

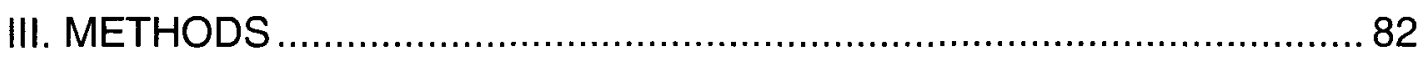

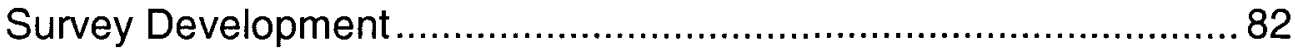

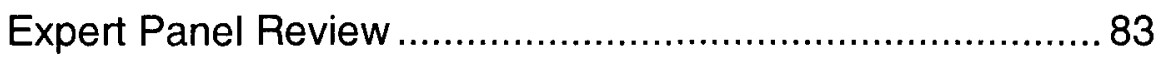

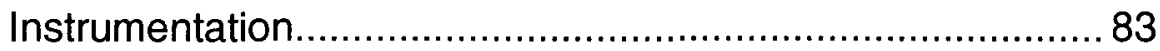

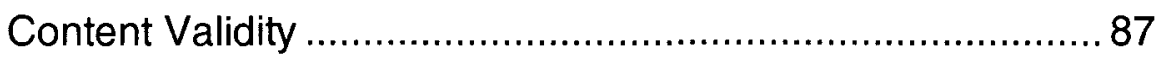

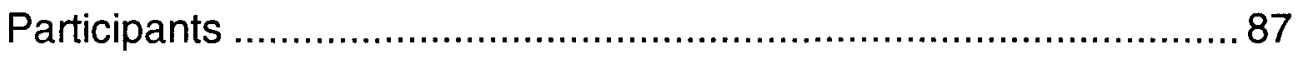

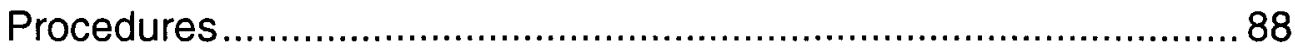

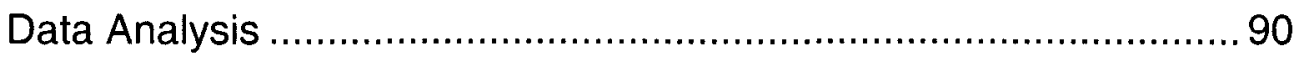

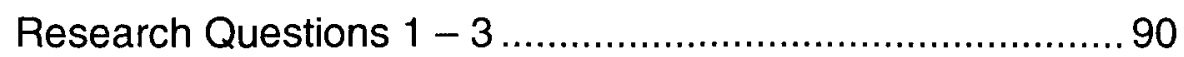

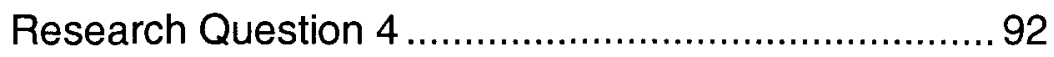

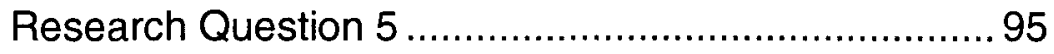

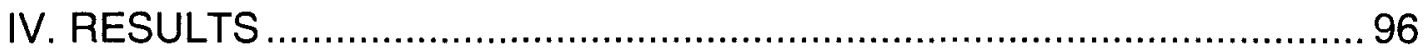

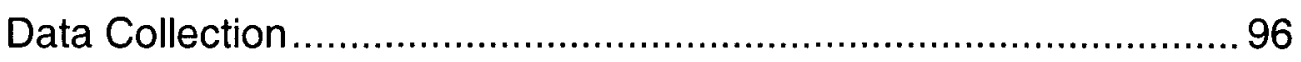

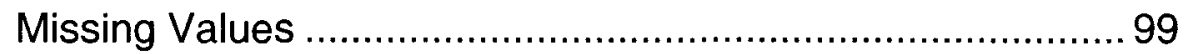

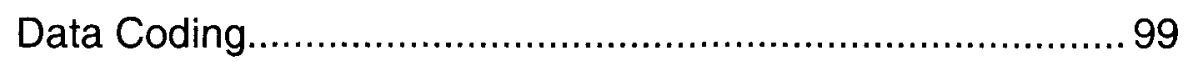

Quantitative Information..........................................99

Qualitative Information .............................................99

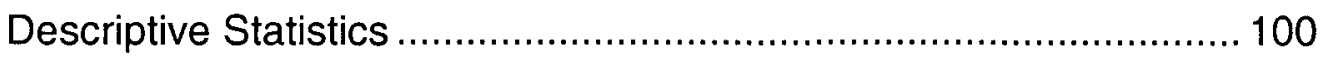

Key Demographic Variables .............................................. 100

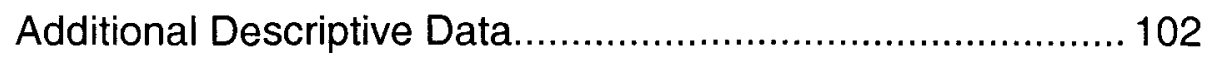

Instrument Validity and Reliability ............................................... 112 
Instrument Validity ............................................................... 113

Instrument Reliability ....................................................... 113

Results of Research Questions .....................................................115

Research Questions 1 - 3: MANOVA................................... 115

Assumptions of MANOVA ........................................ 116

Main Effect.......................................................... 117

Individual Dependent Variables ................................. 118

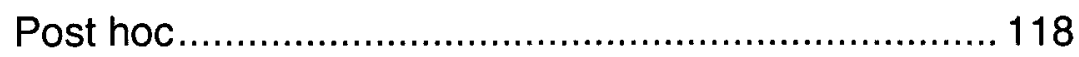

Research Question 4: Independent $t$ - tests ....................... 121

Demographic variable gender................................... 122

Demographic variable pediatric specialization ............. 122

Demographic variable rotation completion.................. 123

Research Question 5: Informal Qualitative....................127

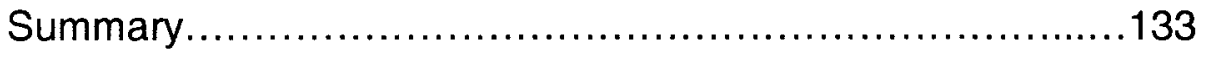

Quantitative.............................................133

Qualitative................................................136

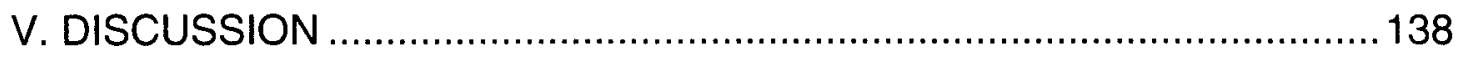

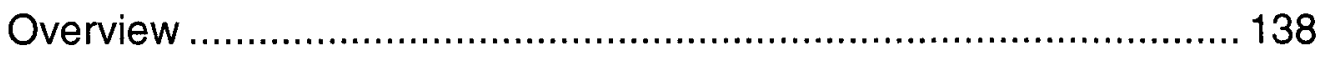

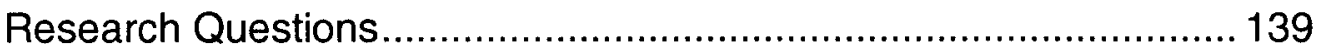

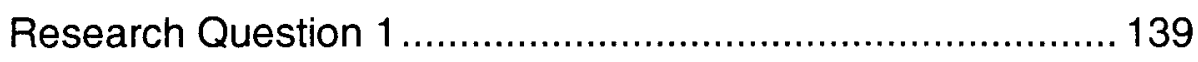

Research Question 2 .................................................... 141

Research Question 3...................................................... 143

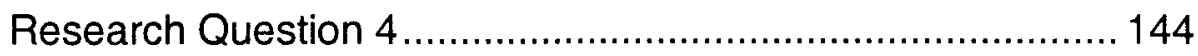


Independent variable gender

Independent variable pediatric specialization ............. 144

Independent variable rotation completion ................... 145

Independent variable educational methods ................ 148

Independent variable educational time ....................... 148

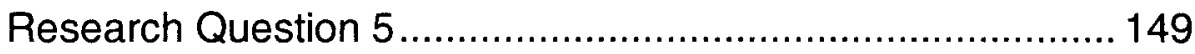

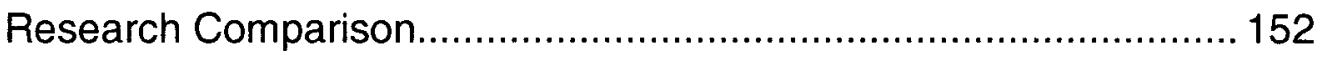

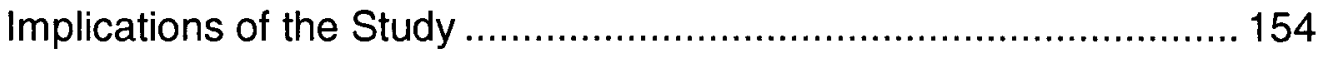

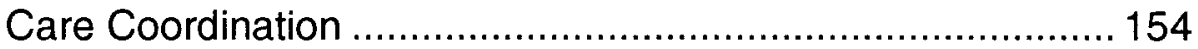

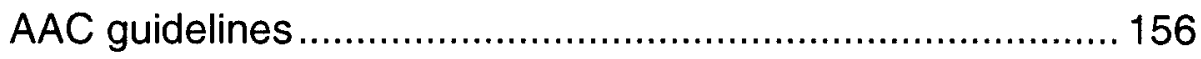

Standards for Hospitals and Skilled Nursing Facilities........... 157

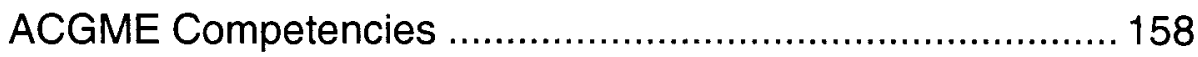

Current Educational Practices ............................................ 160

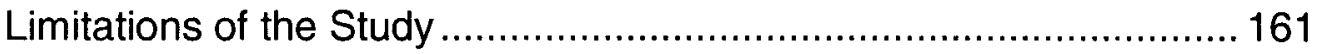

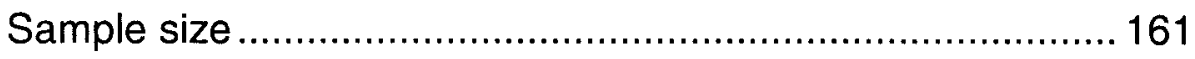

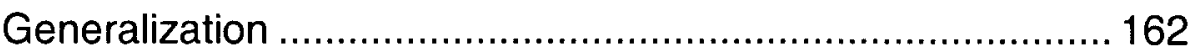

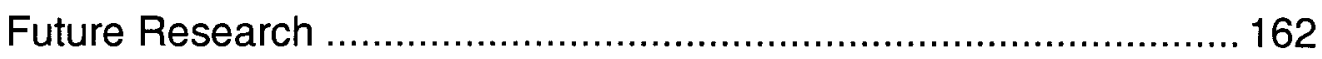

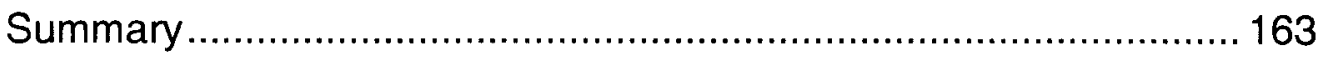

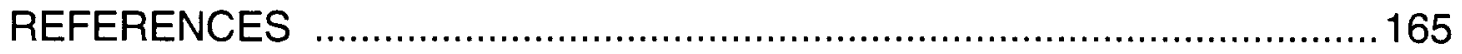

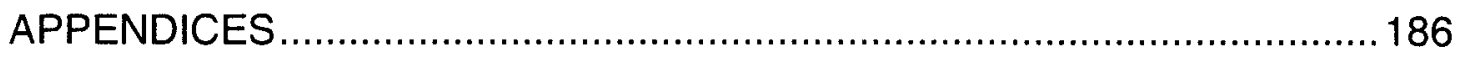

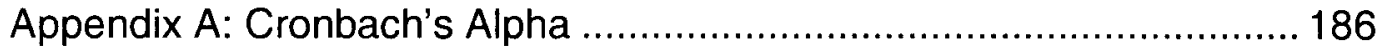

Appendix B: Dependent Variable Histograms ..................................... 192

Appendix C: Demographic Variables .................................................. 196 
Appendix D: Survey of Pediatric Residents: Communication

Disorders and Augmentative and Alternative

Communication (AAC): Preamble

Appendix E: Survey of Pediatric Residents: Communication

Disorders and Augmentative and Alternative

Communication (AAC): Survey Final Expert Panel Copy...................238

Appendix F: Survey of Pediatric Residents: Communication

Disorders and Augmentative and Alternative

Communication (AAC): Survey Monkey Copy ................................24

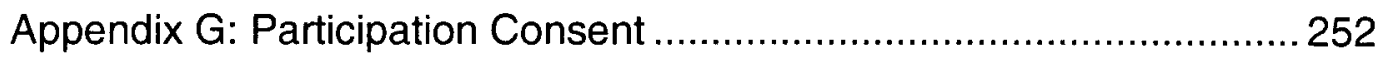

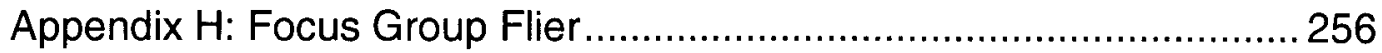

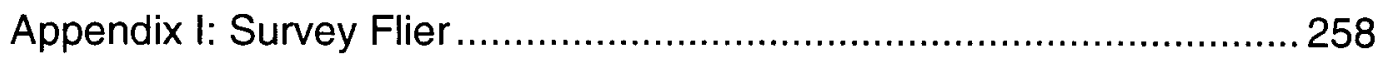

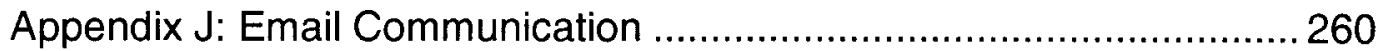

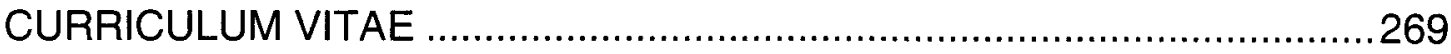




\section{LIST OF TABLES}

\section{TABLE}

1. Survey Questions Aligned with Research Questions............................. 84

2. Study Response Rate/ Per Request .................................................. 97

3. Descriptive Statistics for Gender and PLs............................................ 101

4. Descriptive Statistics for Specialization................................................. 102

5. Descriptive Statistics for Specific Specializations .................................. 103

6. Descriptive Statistics for Rotation Completion ….................................. 104

7. Descriptive Statistics across PLs for Rotation Completion...................... 107

8. Descriptive Statistics for Education: Educational Discussion ................. 109

9. Descriptive Statistics for Education: Educational Method …...................111

10. Descriptive Statistics for Education: Hours Communication Disorders and AAC

11. Cronbach's Alpha Internal Consistency Reliability Coefficients: Study Constructs.

12. Correlations Between Constructs........................................................... 114

13. Main Subject Effects for Education, Knowledge and

Professional Practice

14. Between Subject Marginal Means for PLs across Education, Knowledge and Professional Practice.

15. Bonferroni Multiple Comparisons across PLs for Education, Knowledge, and Professional Practice 120

16. Summary of Rotation $t$ - tests. 124 
17. Summary of Educational Method's $t$ - tests ....................................... 125

18. Correlations Between CD Hours and Constructs ................................ 126

19. Correlations Between AAC Hours and Constructs .............................. 127

20. Psychometric Analysis for Medical Education.................................... 188

21. Psychometric Analysis for Medical Knowledge .................................. 189

22. Psychometric Analysis for Professional Practice ................................ 190

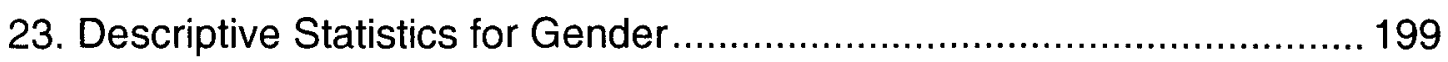

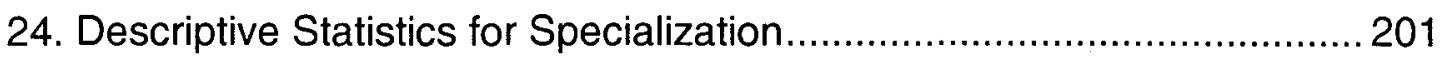

25. Descriptive Statistics for Adolescent Medicine .....................................203

26. Descriptive Statistics for Ambulatory Pediatrics ....................................205

27. Descriptive Statistics for Child Development.....................................207

28. Descriptive Statistics for Community Health Programs .........................2208

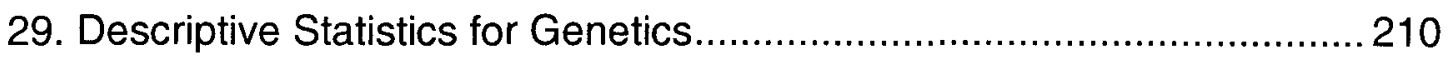

30. Descriptive Statistics for In-Patient Service (Wards) ...........................212

31. Descriptive Statistics for Pediatric Intensive Care Unit .........................214

32. Descriptive Statistics for Private Practitioner's Office............................215

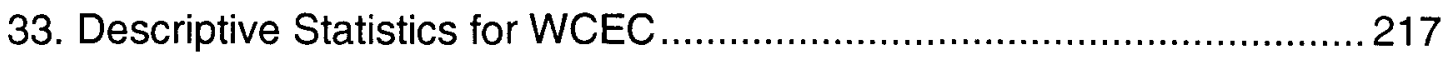

34. Descriptive Statistics for Education: Rotation.........................................220

35. Descriptive Statistics for Education: Ambulatory Pediatric Rotation....... 222

36. Descriptive Statistics for Education: Subspecialized Rotation................225

37. Descriptive Statistics for Education: Morning Report ........................... 227

38. Descriptive Statistics for Education: Core Conference .......................... 230

39. Descriptive Statistics for Education: Didactics ................................... 232 


\section{LIST OF FIGURES}

\section{FIGURE}

PAGE

1. Histogram of frequencies for medical education competence for pediatric levels one, two and three

2. Histogram of frequencies for medical knowledge competence for pediatric levels one, two and three

3. Histogram of frequencies for professional practice competence for pediatric levels one, two and three 


\section{CHAPTER I \\ INTRODUCTION}

Communication is an important aspect of human life. Some scientists say human dominance over animals is due to language (Chomsky, 1968; Fromkin, Rodman, \& Hyams, 2003). We dominate because we think and relate to others using words (Chomsky, 1968; Fromkin, et al., 2003). Communication allows us to connect with each other as individuals and as groups. It is the 'foundation for most social interaction' (Goldstein, Kaczmarek \& English, 2002, p. 27). It is also recognized as the driving force behind personal, corporate and national development and success (D'Aprix, 1982; Houser, Horan, \& Furler, 2008; Marshall \& Heffes, 2006).

With communication as a key and central feature to daily life, its impairment or absence would cause considerable harm (Brinton \& Fujiki,1989; Goldstein, et al., 2002; Rossetti,1996). With impaired or absent communication, participation in general society would be challenging at best (Brinton \& Fujiki, 1989; Goldstein, et al., 2002; Rossetti, 1996). Specifically, the ability to "acquire goods, services and information and make public our thoughts and feelings" ( $p$. 27) would be difficult if not impossible (Goldstein, et al., 2002). Thus, socialization with co-workers, friends, salespeople, educators and loved ones would also be significantly and negatively impacted (Brinton \& Fujiki, 1989; 
Goldstein, et al., 2002; Rossetti, 1996). The problem effecting information exchange is known as communication disorder(s).

\section{Communication Disorders}

Communication disorders affect a significant population of individuals. Currently one out of every ten individuals in the United States experiences some form of communication impairment throughout their lifetime (National Information Center for Children and Youth with Disabilities, 2009; U.S. Department of Education, 2008; U.S. Department of Education, 2008). These individuals face any number of difficulties in their "ability to receive, send, process, and comprehend concepts or verbal, nonverbal and graphic symbol systems" (American Speech-Language-Hearing Association [ASHA], 1993, para. 2).

While one's ability to communicate has been established as one of the highest priorities for business, education and personal relationships, it stands to reason that the need to address one's inability to use functional communication skills to meet the demands of society should be a high priority. Thus, the need for intervention for individuals with communication disorders is important (D'Aprix, 1982; Houser, et al., 2008; Marshall \& Heffes, 2006).

There are many strategies and interventions used to address a person's communication disorder(s). The professionals charged with restoring abilities to individuals experiencing communication disorders are speech-languagepathologists (SLP). These individuals must receive a master's level training in communication disorders at an accredited university followed by a clinical fellowship year with a certified speech-language pathologist. To legitimately 
practice, a SLP must then be licensed by his or her professional organization, the American Speech-Language-Hearing Association (ASHA) and by the state. The licensed SLPS address the broad range of human communication and its disorders (ASHA, 1997). SLPs also provide services across the lifespan in diverse locations such as hospitals, schools and nursing homes.

The communication impairments treated by SLPs can be condensed into two categories, speech disorders and language disorders. By definition speech disorders are impairments of speech sounds, the flow of speech or the production of voice quality (ASHA, 1993). The National Institute on Deafness and Other Communication Disorders (NIDCD), a branch of the National Institute of Health (NIH) report eight to nine percent of children demonstrate speech disorders (NIDCD, 2010).

The other category, language disorders, involves difficulty with the "understanding or use of spoken, written and/or other symbol systems" (ASHA [Data file], 1993). Between six and eight million people in the U.S. are reported as having language impairments (NIDCD, 2002).

As with all disabilities, speech and language disorders can occur together or separately (ASHA, 1997; DSM-IV-TR, 2000). The problem can be mild or severe (ASHA, 1997; DSM-IV-TR, 2000). Communication disorders can also occur in conjunction with a variety of other congenital or acquired disabilities such as mental retardation, cerebral palsy, amyotrophic lateral sclerosis (ALS), or other medical and developmental conditions (ASHA, 1997; DSM-IV-TR, 2000). 


\section{Augmentative and Alternate Communication (AAC)}

For some individuals, despite intervention, it is clear that their degree of impairment will never allow for functional verbal communication (Hill, 2004). As a result of this condition, SLPs address this specific need through the use of augmentative and alternative communication (AAC). Specifically, AAC systems attempt to compensate and facilitate, temporarily or permanently, for the impairment and disability patterns of individuals with severe expressive and/or language comprehension disorders (ASHA, 2004, p.1).

Just as with all other communication disorders, individuals who use or need AAC come from all socioeconomic, ethnic, and racial backgrounds (ASHA, 1993; Buekelman \& Mirenda, 2007; Hill, 2004). Individuals with communication disorders may range in age from infants to geriatrics, and may be diagnosed with a variety of disabilities (ASHA, 1993; Buekelman \& Mirenda, 2007; Hill, 2004).

The common thread between these individuals is verbal abilities that are inadequate to meet all their communication needs (ASHA, 1993, Hill, 2004; Buekelman \& Mirenda, 2007).

Two percent of the population with communication disorders requires augmentative and alternative communication (AAC) systems (ASHA, 1993). However, a quarter of these individuals never receive AAC systems (ASHA, 1993). To quantify this significant group, with the current estimate of the US population at $308,461,257(1-10-2010)$, roughly 61,6923 individuals need AAC systems and 15,4230.6 individuals do not have the system that is needed. While the number of individuals in need of AAC is large, it should be noted that the 
population is growing. For example, the number of children with such communication needs is increasing due to several factors. Survival rates for at risk births continue to rise (Martin, Kung, Mathews, Hoyert, Strobino, Guyer, \& Sutton, 2006) as is the rate of children diagnosed on the autism spectrum disorder (ASD) (Center for Disease Control, 2010). The Center for Disease Control (CDC) estimates an average of 1 in 110 children in the United States has an autism spectrum disorder (2010). In 2011 alone, the CDC estimates approximately 36,500 children will be diagnosed with an ASD. Even though the ASD population in need of services for a communication disorder is already significant, the CDC goes on to state that 40 percent of children with an ASD do not have verbal communication (2010). Therefore, of the estimated 36,500 children that will be identified with an ASD this year, 14,600 will be unable to verbally communicate and will need some form of augmentative or alternative communication system.

\section{Identification and Intervention Services}

The importance of and need for intervention services for individuals with communication disorders is apparent. The current population requiring services for communication impairments is significant and continuing to rise (Center for Disease Control, 2010; Martin, et al, 2006). Compounding the issue are obvious shortfalls in the provision of services, especially regarding to AAC (American Speech-Language and Hearing Association, 1993).

There may be many reasons for the lack of identification and implementation of $A A C$ systems. For example, training regarding $A A C$ for 
professionals in educational agencies, medical agencies and/or adult service agencies may not be required or emphasized (Bailey, Stoner, Parette, \& Angell, 2006; Bingham, Spooner, \& Browder, 2007; Ratcliff, Koul \& Lloyd, 2008; Snell, Chen \& Hoover, 2006; Sneed, May, \& Stencel, 2004). Specifically, within the field of medicine, a lack of training may be due to oversight within pediatric resident education (Sneed, May, \& Stencel, 2000; Sneed, May, \& Stencel, 2001; Sneed, et al., 2004; Sneed, May, Stencel, \& Paul, 2002).

\section{Pediatrician's Role}

Pediatricians have a vital role in a child's life. With regard to children with communication disorders and needing augmentative and alternative communication, the journey to receiving help often begins with a trip to the pediatrician's office. According to the American Medical Association (AMA) (2008), due to their training and concern for a child's overall well-being, pediatricians are uniquely qualified to provide such care and service. By definition pediatricians practice the "specialty of medical science concerned with the physical, emotional, and social health of children from birth to young adulthood" (Goodman, 2005, p. 56). Pediatric care encompasses a broad spectrum of health services ranging from preventive health care to the diagnosis and treatment of acute and chronic diseases. Pediatricians must understand the factors that affect the growth of children as it corresponds to their current stage of physical and mental development (Goodman, 2005). These professionals, therefore, play a substantial role in the ongoing care of children, especially those with disabilities. Therefore, the primary care pediatrician plays an important role 
in the effort to provide appropriate services for communication disorders and augmentative and alternative communication disorders (Desch, Gaebler-Spira, \& the Council on Children with Disabilities, 2008).

Pediatricians are charged to recognize communication disorders and/or the need for AAC and make the appropriate referrals. Pediatricians then 'develop a care-coordination process that involves all available resources to help families through the often complicated processes of healthcare (Desch, et al., 2008 p. 1275).

\section{Healthcare Standards for Pediatrics and AAC}

Part of the complicated healthcare process was formally addressed, in July of 2008 when a new initiative within the realm of pediatrics was released (Desch, et al., 2008). At that time a clinical report regarding the prescription of assistive technology systems with a focus on children with communication disorders was published in the journal, Pediatrics (Desch, et al., 2008).

This clinical report added standards of treatment specifically for children with communication disorders needing AAC. These standards stated that pediatricians are to be informed advocates regarding augmentative and alternative communication (Desch, et al., 2008). Pediatricians also have a critical role in the provision of AAC because caregivers, patients or allied health professionals may request their referrals, opinion, sign prescriptions or letters of medical necessity to help obtain funding both for the device and the assessment for some of these systems (Desch, et al., 2008). Within the report all pediatricians, including sub-specialties vital to medical home, should work 
cooperatively and collaboratively to improve appropriate access to AAC devices and programs (Desch, et al., 2008).

\section{Professional Training}

The substantial responsibilities of pediatricians require extensive training to fulfill their roles in the lives of children. This is the job of medical education, and specifically the goal of resident education. To ensure the best educational outcomes for today's professionals, the Accreditation Council for Graduate Medical Education (ACGME) and the American Board of Medical Specialties (ABMS) jointly developed a long-term initiative, the Outcome Project (ACGME, 2006).

The ACGME Outcome Project developed two goals: 1) to make sure residency program content meets the changing needs of today's health care system, and 2) to establish valid outcome assessment systems to measure a programs' educational effectiveness (ACGME, 2006 [Data file]). To meet these goals, the ACGME identified six competencies based on a national consensus on "what residents should know and be able to do" for certification (ACGME, 2006, n.p; Joyce, 2006). These competencies are also for the maintenance of certification of current physicians (Joyce, 2006). The six competencies "ensure that residents develop competence as physicians in order to complete their training and competently practice as independent practitioners" (Joyce, 2006, p. 10). The six domains of the ACGME competencies are: Medical Knowledge, Patient Care, Professionalism, Interpersonal and Communication Skills, PracticeBased Learning and Improvement, and System-Based Practice (Joyce, 2006). 
Each competency has specific requirements as well as guidelines for assessment. Some competencies, such as medical knowledge and patient care, have already been established within medical education in some form (Joyce, 2006). These competencies were identified within the ACGME training materials as not needing to be targeted further (Joyce, 2006).

Two of the competencies have been previously identified as part of medical education, but in need of further attention (Joyce, 2006). These included interpersonal skills and communication, as well as professionalism. With regard to interpersonal skills and communication, residents are expected to communicate with patients beyond history taking in a manner that is clear, effective, and empathetic (Joyce, 2006). Professionalism was also targeted for clarification. Residents are to demonstrate respect, compassion, strong ethical principles and sensitivity to diversity (Joyce, 2006).

New expectations for residents include practice based learning and improvement as well as systems-based practice (Joyce, 2006). These competencies not only emphasize continued and life-long improvement of patient care but also familiarity and responsiveness to the larger context, system and resources within the health care community (Joyce, 2006).

Changes in educational methods have been needed, especially regarding communication disorders and augmentative and alternative communication (Sneed, et al., 2000; Sneed, et al., 2004). Previous research on the education of pediatric residents found little literature describing the preparation of pediatricians to prescribe therapies and devices to children with disabilities (Sneed, et al., 
2000). In 2000 , a survey of residents and pediatricians was conducted to identify current levels of ability with regard to the prescription of therapies and durable medical equipment, such as augmentative and alternative communication (Sneed, et al., 2000). The results of the survey indicated that approximately $70 \%$ of the respondents didn't have training in prescribing certain forms of durable medical equipment and over $50 \%$ had a complete lack of training in prescribing certain therapies (Sneed, et al., 2000). Three-quarters of the respondents indicated that they did not believe that they were adequately prepared to take an active role in prescribing therapies and durable medical equipment (Sneed, et al., 2001). This is despite federal guidelines and the American Academy of Pediatrics (AAP) policy often requiring the prescription of these therapies and devices be initiated and monitored by physicians (Sneed, et al., 2001; Sneed, et al., 2004).

The results of these studies point to possible shortfalls within resident education regarding training for the prescription of therapies, such as speechlanguage therapy, and durable medical equipment, in particular, AAC. The authors of the study stated that there was "a striking sense of inadequate training evidenced among residents as well as practicing physicians ... for the various durable medical equipment categories" (Sneed, et al. 2000, p. 554). If pediatricians, the American Academy of Pediatrics and the federal government believe that pediatricians should have a significant role in the provision and supervision of therapies and AAC, then every effort should be made for their competent participation (Sneed, et al. 2004). 


\section{Statement of the Problem}

Pediatricians play an important role in the lives of children with disabilities. Within their practice, pediatricians are to act as a medical home for children and take on the ethical responsibility to coordinate the care of a child up to age twenty-one (American Academy of Pediatrics [APA], 1999; APA, 2005; Brewer, McPherson, Magrab, \& Hutchins ,1989). Pediatricians must have knowledge of many aspects of a child's development or lack thereof, including a potential need for speech-language therapy and augmentative and alternative communication (AAC). Within the framework of the medical home, the pediatrician then takes on the role of care coordinator. Within care coordination, pediatricians are to identify possible communication disorders followed by an appropriate referral for an evaluation by a certified speech-language pathologist (Desch, et al., 2008).

In the 2008 clinical guide, Pediatrics, Desch, Gaebler-Spira, \& Disabilities, offer the premise that pediatricians should ensure access to appropriate augmentative and alternate communication services, including assessment, training, monitoring and funding. However, it is unknown to what degree pediatricians understand their responsibility. Additionally, it is unclear as to what level pediatricians know or understand augmentative and alternate communication systems and services

A previous assessment of resident knowledge of communication disorders and $A A C$ in 2000 found that the majority of pediatric residents did not have knowledge about speech-language therapy, and they did not feel comfortable in 
prescribing communication devices (Sneed, et al., 2000). In 2004, Sneed, May, \& Stencel also found that pediatricians and pediatric residents were unsure of their role as care coordinators when prescribing therapies and durable medical equipment (DME). Only a minority of the survey respondents fulfilled the expectations of American Association of Pediatrics (AAP) policies (Sneed et al., 2004). Furthermore, the study indicated that physicians prescribed diagnoses and not much else. Reasons for these professional shortcomings included a lack of experience, a lack of education, a lack liability, and a lack of communication within the care coordination team (Sneed, et al., 2004).

To ensure the growing number of children with special health care needs ( $\mathrm{CSHCN})$ is being provided quality health care, it is important to address the question of whether pediatricians are being adequately prepared to assume leadership in prescribing the specialty therapies and durable medical equipment often required by the CSHCN population. As previously noted, Sneed et al. (2004) found substantial gaps in pediatric resident training. These gaps were not only with regard to care coordination within the medical home, but also their knowledge and prescription of therapies and DME. Their findings help to establish the necessity of expanding training programs to ensure quality health care for CSHCN (Sneed et al., 2004). Thus, the critical question is whether medical programs have addressed the need of residents for proper training in identifying communication disorders and the need for AAC. 


\section{Purpose of Study}

There is a compelling need to follow-up previous research to determine how this need is presently being met. This study provided current information about the state of pediatric resident education at one training institution. It investigated residents' abilities to identify and provide ongoing care for children with communication disorders and AAC within the framework of the ACGME competencies. Survey questions focused on 3 major areas of concern: (1) medical resident educational experiences, (2) current knowledge, and (3) professional practice.

The outcomes of this study may benefit a significant number of individuals and groups. It may help the one in ten individuals currently experiencing a communication disorder, and the 1.4 million students with communication disorders served in the public schools' special education programs (Speech and Language, 2008; U.S. Department of Education, 2008). It may support the roughly $61,6922.5$ individuals using AAC systems and 15,4230.6 individuals that do not have the system that is needed. For the 14,600 children identified in 2011 with an ASD who will be unable to verbally communicate it may also provide a better understanding of meeting their needs.

This study may also provide beneficial information for the pediatric residency program here at the University of Louisville. It could confirm that the educational methods are addressing the ACGME competencies, specifically in the area of communication disorders, or it may help target areas that need to be addressed. 


\section{Research Questions}

This study will investigate differences in perceived ACGME competency regarding communication disorders and AAC across the three levels of pediatric residency at the University of Louisville. The following questions were investigated:

1. To what extent are there differences in perceived pediatric resident knowledge of communication disorders and AAC across pediatric levels?

2. To what extent are there differences in perceived pediatric resident competency for professional practice regarding the care of children with communication disorders and AAC across pediatric levels?

3. To what extent are there differences in perceived pediatric resident educational training experiences for communication disorders and AAC across pediatric levels?

4. What effects do demographic variables have on residents' perceived competency?

5. What perspectives do pediatric residents hold regarding communication disorders and $A A C$ as part of their training?

\section{Significance of Research Study}

The completion of this investigation was important for a number of reasons. With the recent and continued transition of the medical educational process to the ACGME competencies, new research is needed to validate student performance within this framework. The ACGME competencies were 
developed to improve the quality of patient care in accordance with professional and ethical policies. The study will identify student's perceived aptitude toward these competencies with regard to communication disorders and AAC.

This study provided updated data on previous research, but in a novel fashion. Previous literature regarding this topic is scarce. The information that has been provided by Sneed, et al. (2000; 2001; 2004), and Sneed, May, Stencel, \& Paul (2002) did not research the specific topic of AAC, nor did it research either communication disorders or AAC in relation to the ACGME competencies.

\section{Conclusion}

Communication is an important key feature to every aspect of life. An impairment in communication abilities can cause significant harm to an individual's participation and socialization within their community, career, educational instruction and personal relationships. Communication disorders affect a significant number of individuals within the United States. A considerable group within this population demonstrates a type and degree of impairment that does not allow for verbal exchange as a viable means for communication. These individuals rely on the use of AAC to provide either an augmentation of their natural speech or an alternative means for expression.

It has been demonstrated that children with general communication impairments as well as complex communication needs rely on pediatricians to prescribe the services of speech language pathologists. It is therefore important to ascertain whether medical residents are receiving the necessary training in 
their educational program to fulfill their role. In light of the new ACGME competencies, it is important to understand how pediatric residents perceive their current level of abilities.

This study investigated the current level of perceived competency of residents within and across pediatric levels. Survey questions will focus on 3 major areas of concern: 1) medical resident educational experiences, 2) current knowledge and competencies, and 3) medical residents' personal opinions about their educational preparation to address these issues. The data were analyzed for significant changes in perceived competency of pediatric residents regarding their medical knowledge, professional practice and education regarding communication disorders and AAC over the course the three-year pediatric residential program at the University of Louisville. Further analysis were conducted to then ascertain if there are significant difference between their rating of communication disorders and $A A C$ within their medical knowledge, professional practice and education.

This study may also provide beneficial information for the pediatric residency program here at the University of Louisville. It will confirm that the educational methods are addressing the ACGME competencies, specifically in the area of communication disorders, or it may help target areas that need to be addressed. Either outcome for this study will serve as a benchmark of educational inquiry for the high educational standards here at the University of Louisville. 


\section{Definition of Terms}

In the context of this study, the following definitions have been operationally defined:

\section{Accreditation Council for Graduate Medical Education (ACGME)} competencies. Six competencies for the certification and maintenance of certification of doctors (ACGME, 2006). These competencies 'ensure that residents develop competence as physicians in order to complete their training and competently practice as independent practitioners' (ACGME, 2006).

Medical knowledge. Residents must demonstrate knowledge about established and evolving biomedical, clinical, and cognate (e.g. epidemiological

and social-behavioral) sciences and how to apply this knowledge to patient care (ACGME, 2006).

Patient care. Residents must be able to provide patient care that is compassionate, appropriate, and effective for the treatment of health problems and the promotion of health (ACGME, 2006).

Professionalism. Residents must be able to investigate and evaluate their patient care practices, appraise and assimilate scientific evidence, and improve their patient care practices (ACGME, 2006).

Interpersonal and communication skills. Residents must be able to demonstrate interpersonal and communication skills that result in effective information exchange and teaming with patients, their patients families, and professional associates (ACGME, 2006). 
Practice-based learning and improvement. Residents must be able to investigate and evaluate their patient care practices, appraise and assimilate scientific evidence, and improve their patient care practices (ACGME, 2006).

System-based practice. Residents must demonstrate an awareness of and responsiveness to the larger context and system of health care and the ability to effectively call on system resources to provide optimal health care (ACGME, 2006).

Augmentative and alternative communication. Attempt to compensate and facilitate, temporarily or permanently, for the impairment and disability patterns of individuals with severe expressive and/ or language comprehension disorders (ASHA, 1993). AAC may be required for individuals demonstrating impairments in gestural, spoken, and/or written modalities (ASHA, 1993).

Autism spectrum disorder. A neurodevelopmental disorder that has three core features: impairments in social interaction, impairments in verbal and nonverbal communication, and repetitive, restricted, and stereotyped interests and patterns of behavior (American Psychiatric Association, 2000). ASD includes the following: Autistic disorder, pervasive developmental disorder-not otherwise specified (PDD-NOS), Asperger syndrome, Rett syndrome, and childhood disintegrative disorder (Mirenda \& lacono, 2009).

Care coordination. A system of improving the quality of services for children with special health care needs (CSHCN). It links children and their families with appropriate services and resources in a coordinated effort to achieve good health (AAP, 2002). 
Children with Special Health Care Needs (CSHCN). Children with special health care needs (CSHCN) are defined by the Department of Health and Human Services, Health Resources and Services Administration, Maternal and Child Health Bureau (MCHB) as: "...those who have or are at increased risk for a chronic physical, developmental, behavioral, or emotional condition and who also require health and related services of a type or amount beyond that required by children generally" (McPherson, Arango, Fox, Lauver, McManus, Newacheck, Perrin, Shonkoff, \& Strickland, 1998, pg 137).

Common program requirements. The set of ACGME requirements that apply to all specialties and subspecialties (ACGME, 2009).

Communication disorder. A communication disorder $(C D)$ is "impairment in the ability to receive, send, process, and comprehend concepts or verbal, nonverbal and graphic symbol systems." Communication disorders include the realms of speech or language disorders that are congenital or acquired (ASHA, 1993, p. 1).

Competencies. The specific knowledge, skills, behaviors and attitudes and the appropriate educational experiences required of residents to complete graduate medical education programs at an accredited university (ACGME, 2009).

Complex communication needs. Individuals who rely on AAC when they cannot meet their communication needs through the current method of communication (Justice, 2006; Hill, 2004). 
Graduate medical education. The period of didactic and clinical education in a medical specialty which follows the completion of a recognized undergraduate medical education and which prepares physicians for the independent practice of medicine in that specialty, also referred to as residency education (ACGME, 2009).

Graduate-year level. A resident's current year of accredited graduate medical education. Within the University of Louisville is referred to as the Pediatric Level (PL).

Education. Training and instruction in a particular subject, or the imparting and acquiring of knowledge through teaching and learning (Encarta $\circledast$ World English Dictionary [North American Edition], 2009 [data file]).

Knowledge. It is general awareness or possession of information, facts, ideas, truths, or principles. It can also be seen as understanding or awareness of specific information or a proficiency in all that can be known regarding particular topic Encarta ${ }^{\circledR}$ World English Dictionary [North American Edition], 2009.

Medical home. A "partnership approach with families to provide primary health care that is accessible, family centered, coordinated, comprehensive, continuous, compassionate and culturally effective" (Sia, et al., 2004; AAP, 2002).

Outcome project. The ACGME developed a long-term initiative, the Outcome Project, which increased emphasis on educational outcomes in the accreditation process of residency education programs (ACGME, 2009). 
Professional practice. The American Board of Pediatrics (ABP) developed guidelines for the teaching and evaluation of professionalism, or professional practice, as part of the core curriculum for residency training in pediatrics (Fallat, Glover \& and the Committee on Bioethics, 2007, pg. e1124). These guidelines overlap and accentuate five of the six ACGME competencies including the following: Patient Care, Professionalism, Interpersonal and Communication Skills, Practice-Based Learning and Improvement and SystemBased Practice. Professional practice is distinctive from medical knowledge. The following eight components are endorsed by the ABP: honesty and integrity, reliability and responsibility, respect for others, compassion/ empathy, selfimprovement, self-awareness/ knowledge of limits, communication and collaboration and altruism and advocacy (Fallat, et al., 2007).

Patient. "A recipient of a health care service or a client in a health care service (Mosby's Medical Dictionary, 8th edition, 2009, p. 7).

Pediatricians. Pediatricians practice the "specialty of medical science concerned with the physical, emotional, and social health of children from birth to young adulthood" (American Medical Association (AMA), 2008).

Program. A structured educational experience in graduate medical education designed to conform to the Program Requirements of a particular specialty/subspecialty, the satisfactory completion of which may result in eligibility for board certification (ACGME, 2009).

Resident. A physician in an accredited graduate medical education specialty program (ACGME, 2009). 
Residency. The AMA identifies residency as 'the period of training in a specific medical specialty (2008)'. Medical residency occurs after a student completes four years of undergraduate and pre-medicine training and then graduates from four years of medical school (AMA, 2008). Residents typically have three years, or pediatric levels (PL), within their residency.

Rotation. An educational experience of planned activities in selected settings, over a specific time period, developed to meet goals and objectives of the program (ACGME, 2009). 


\section{CHAPTER II}

\section{REVIEW OF LITERATURE}

The purpose of this chapter is to provide a review of literature found to be pertinent to this study. Five major areas are addressed: (a) historical chronology of communication disorders, (b) historical chronology of augmentative and alternative communication, (c) historical chronology of pediatric residency, (d) current pediatric resident practice and competencies, and (e) current professional expectations of pediatric resident regarding communication disorders and augmentative and alternative communication.

\section{Historical Chronology of Communication Disorders}

The topics of communication disorders, medicine and augmentative and alternative communication date back to ancient history. These three areas are therefore not new. Over the years, cultural beliefs, scientific inquiry and philosophies have influenced their development and ultimately their practice. To understand their current state, a brief review of each discipline's recent history is warranted.

\section{Early Development}

In looking at the development of the field, speech-language pathology strongly parallels the development of medical education. In fact, the "Father of Medicine," Hippocrates (c. $460-357$ B.C.) is credited with being one of the first 
to document several communication disorders including aphasia and stuttering (Klingbeil, 1939).

History is largely silent regarding communication disorders until the $19^{\text {th }}$ century. As in the development of medicine, there were social and cultural movements that brought about the formation of professional speech-language pathologists (Bryant, \& Bryant, 2003). Within this century, there was a significant increase in services for individuals with disabilities (Bryant, \& Bryant, 2003). Examples of these include the works of Thomas Hopkins Gallaudet with individuals with hearing disabilities and Louis Braille who created a tactile system of reading for individuals who were blind (Bryant, \& Bryant, 2003).

The first period in the history of communication disorders, the elocution movement, also began in the early nineteenth century (Duchan, 2002). This movement can be understood in the context of the movie Pygmalion or the more recent book and Broadway play, My Fair Lady. During their time, elocutionists worked to improve individual's public speaking and communication abilities.

The study of communication disorders in the $19^{\text {th }}$ century originated in Europe and then immigrated to the United States. A significant number of individuals in Europe began applying scientific inquiry towards communication problems (Duchan, 2002; Klingbeil, 1939). American individuals would study under the expertise of European physicians and then bring their knowledge back to the States (Duchan, 2002). These scientists came from a variety of fields including medicine and education. The study of communication problems also attracted a number of self-styled healers who offered a number of home-grown 
remedies (Duchan, 2002;Klingbeil, 1939). An assortment of notable scientists investigated communication disorders during the $19^{\text {th }}$ century. A few of these individuals were Erasumus Darwin, Robert James Graves, Alexander Melville Bell, Jean Baptiste Bouillaud, Paul Broca and Carl Wernicke (Duchan, 2002; Klingbeil, 1939).

Using the information available to them, these scientists attempted to identify the cause for different communication disabilities. Within the $19^{\text {th }}$ century, the typical etiologies were identified as either biological or environmental (Duchan, 2002). Environmental interventions for speech errors were addressed through lifestyle. For example, the scientists would look at the person's personal hygiene and moral conduct (Duchan, 2002), whereas, biological causes were assessed using scientific inquiry and based on the growing fields of anatomy and physiology (Klingbein, 1939).

As the $20^{\text {th }}$ century began, the early foundation of communication disorders was developing within the realm of education. During the $20^{\text {th }}$ century, a number of individuals were interested in curing various communication disorders (Duchan, 2002). One reason for this interest was the child labor laws and compulsory education laws being enacted during the American Industrial Revolution (Duchan, 2009; Kleeck \& Schuele, 2010; Moore, 1939). Educators found themselves responsible for the education of children with a wide variety of disabilities including communication disorders (Duchan, 2009; Moore, 1939). Interestingly, Chicago was the first city to respond to the needs of its educators with regard to communication disorder training (Duchan, 2009; Moore, 1939). In 
response to a district-wide survey, ten educators that were specialized in the correction of speech defects were commissioned to provide services to 1,287 children (Duchan, 2009; Moore, 1939). These early speech pathologists were commissioned to serve child with "stuttering and stammering, lisping and lalling, thick speech, motor aphasia, mutism and nasality" (Duchan, 2009, para. 1).

The use of educators that specialized in speech correction quickly spread across the nation. With the increase in this specialized profession came the natural desire to meet with other like-minded individuals and receive continuing education. In 1915, the National Association of Academic Teachers of Public Speaking met (Duchan, 2009; Moore, 1939) and began to formally distinguish itself from the arenas of general communication, education, and medicine (Duchan, 2002).

In 1925, members of the National Association of Academic Teachers of Public Speaking founded the American Speech-Language Hearing Association (ASHA) (ASHA, 1993; Duchan, 2002; Moore, 1939). Speech-language pathology was well on its way to growing as a professional field of study.

\section{Current Definitions in Communication Disorders}

Today, the field of communication disorders is recognized as an established area of clinical profession, education and research. ASHA has continued to govern speech-language-pathologists since the first meeting in 1925. By current accounts, ASHA has grown from its original eleven individuals to comprise 140,000 active members serving throughout America. 
The definition of communication disorders has likewise evolved. It has broadened from concepts such as stuttering and stammering (Duchan, 2009) into a research-based classification. According to the American Speech-LanguageHearing Association (ASHA) (1993), a communication disorder is "impairment in the ability to receive, send, process, and comprehend concepts or verbal, nonverbal and graphic symbol systems."(n.p., para 1) The Diagnostic and Statistical Manual, Forth Edition, defines communication disorders as "mental disorders of childhood affect listening, language and speech" (DSM-IV-TR, 2000, p. 58). Communication disorders are recognized as occurring throughout the lifespan and being congenital or acquired (ASHA, 1993; DSM-IV-TR, 2000).

The field of speech-language pathology encompasses all aspects of a communication message including; phonology, morphology, pragmatics, semantics, syntax, speech and voices issues, as well as feeding, swallowing and motor speech disorders. To simplify the complex concept of communication disorders, two specific areas, speech disorders and language disorders are defined. A language disorder involves difficulty with the "understanding or use of spoken, written and/or other symbol systems" (ASHA, 1993, n.p., I-B).

Between six and eight million people in the U.S. are reported as having language impairments (NIDCD, 2002). Language disorders can be further divided into problems with the form of language, the content of language or the function of language. The form of language can be thought to include the sound system rules (phonology), the rules for the use of word forms (morphology) and the rules for combining words into sentences (syntax) (ASHA, 1993). The content of 
language, or semantics, consists of the rules for word or sentence meanings (ASHA, 1993). Finally, the function of language is its appropriate application across a variety of social contexts (ASHA, 1993).

Speech disorders are impairments of the sounds of speech, fluency or flow of speech or the production of voice quality (ASHA, 1993). The National Institute on Deafness and Other Communication Disorders (NIDCD), a branch of the National Institute of Health $(\mathrm{NIH})$ reported that eight to nine percent of children demonstrate speech disorders (NIDCD, 2010).

A communication disorder may be the sole difficulty that a child may be experiencing, or it may be in combination with a variety of other disabilities (ASHA, 1993; DSM-IV-TR, 2000; NIDCD, 2002). It can be mild to profound and individuals can have one or any combination of communication disorders. Thus receiving a diagnosis of a communication disorder may have a range of implications (ASHA, 1993; DSM-IV-TR, 2000; NIDCD, 2002). As stated by ASHA (1991), "communication is the essence of human life and all people have the right to communication to the fullest extent possible" (n.p., para 2). Communication disorders affect a person's emotional and social life, and can compromise educational and occupational success (D'Aprix,1982; Houser, Horan, \& Furler, 2008; Marshall \& Heffes, 2006; NIDCD, 2002). Thus, the cost of communication disorders on person's quality of life and potential can be substantial.

The potentially adverse effects of communication disorders are experienced by a significant population. One of every ten people in the United 
States experience some type of communication disorder (Speech and Language, 2008; Twenty-Sixth Annual, 2008). Within that number are 1.4 million students served in the public schools' special education programs (Speech and Language, 2008; U.S. Department of Education, 2008).

The number of people experiencing communication disorders is on the rise. This increase is due to improved survival odds for medically fragile infants, significant injuries, acquired diseases, aging (NIDCD, 2002; Martin, Kung, Mathews, Hoyert, Strobino, Guyer, \& Sutton, 2006) and children diagnosed with autism spectrum disorders (NIDCD, 2011).

\section{Historical Chronology of Augmentative and Alternative Communication}

As the field of communication disorders continued to develop, it became clear that some disorders would never allow individuals to experience verbal communication (Hill, 2004). Augmentative and alternative communication (AAC) methods were developed to help individuals successfully communicate. The discipline of AAC has grown within the last forty years with the advancement of

recent technology (Higginbotham, Shane, Russell, \& Caves, 2007). Although AAC has experienced a recent birth as a discipline in the United States, its history is an old one closely associated with the histories of assistive technology and disabilities (Bryant, \& Bryant, 2003).

\section{Assistive Technology}

Since ancient times, assistive technology has been used to compensate for functional limitations due to disabling conditions (Bryant, \& Bryant, 2003; Hill, 2004; Moser, O'Neill, Oyer, Wolfe, Abernathy \& Schowe, 1960; Zangari, Lloyd, \& 
Vicker, 1994). Assistive technology can be defined as any tool "to improve the skills, abilities, lifestyle, and independence of individuals' with acquired or congenital disabilities" (Glennen, \& DeCoste, 1997, p. 6). Assistive technology helps individuals minimize their 'disability' and live more functional, independent lives (Galvin \& Scherer, 1996).

AAC is included under the umbrella of assistive technology (Glennen, \& DeCoste, 1997). AAC systems attempt to compensate and facilitate, temporarily or permanently, for the impairment and disability patterns of individuals with severe expressive and/or language comprehension disorders (ASHA, 1993; ASHA, 2004). AAC may be required for individuals demonstrating impairments in gestural, spoken, and/or written modalities (ASHA, 1993; 2005).

\section{Early History}

As with assistive technology, the history of AAC in the United States is embedded within the history of disabilities or communication need. The use of AAC systems predates written historical records (Glennen, \& DeCoste, 1997; Hill, 2004; Moser, et al., 1960; Zangari, et al., 1994). One example is the use of American Indian Hand Talk. This form of sign language was used by American Indian tribes to overcome language barriers for trade and other communication needs (Beukleman, \& Mirenda, 2005; Childress, 2002; Moser, et al., 1960). After the colonization of North America by various immigrant communities, the history of assistive technologies as well as AAC is largely silent and not formally addressed until the twentieth century. 
Upon review of the literature beginning in the twentieth century, a pattern emerges regarding the development of assistive technology and thus, AAC. An event or catalysis causes an increase in the population of individuals with disabilities. This impetus is followed by several reactions. One reaction was an increased awareness by the general public and hence some form of social movement. Social awareness led to formal legislation by the government to support individuals with disabilities through different means including assistive technology.

The first example of this pattern, and the first formal legislation regarding assistive technology in the United States was after World War I (Bryant \& Bryant, 2003; Childress, 2002; Hill, 2004; Reily, Panhan \& Tupinamba, 2009; United States Department of Veterans Affairs, n.d.). The obvious catalysis was the injuries many soldiers acquired during the war. The government recognized the increase of individuals with disabilities and the subsequent need to help disabled veterans post war (Ability Magazine, 2003; Bryant, \& Bryant B.R., 2003; Childress, 2002; Hill, 2004; Reily, et al., 2009; United States Department of Veterans Affairs, n.d.). Thus, legislation such as the National Defense Act of 1916, the Smith-Hughes Act (P.L. 347) and the Soldier's Rehabilitation Act (also known as the Smith-Sears Veterans Rehabilitation Act in 1918) authorized vocational services for veterans (Ability Magazine, 2003; Bryant, \& Bryant, 2003; Childress, 2002; United States Department of Veterans Affairs, n.d.). This legislation was soon followed by the Smith-Fess Citizen's Rehabilitation Act in 1920 (Ability Magazine, 2003; Bryant, \& Bryant, 2003; Childress, 2002; United 
States Department of Veterans Affairs, n.d.). It extended vocational rehabilitation services and placement services to all Americans with physical disabilities (Ability Magazine, 2003; Bryant, \& Bryant, 2003; Childress, 2002; United States Department of Veterans Affairs, n.d.). Therefore, all Americans with disabilities could be provided training, job adjustment, prosthetics, and job placement (Bryant, \& Bryant, 2003; Childress, 2002; Ability Magazine, 2003). This rehabilitation act also covered the use of seeing-eye dogs and the standardization of Braille (Bryant, \& Bryant, 2003; Childress, 2002; Ability Magazine, 2003; United States Department of Veterans Affairs, n.d.). Then in 1935, a provision within the Social Security Act granted funds to states to help the blind and disabled (Bryant, \& Bryant, 2003). This further promoted the development of devices for those with disabilities (Bryant, \& Bryant, 2003).

The pattern for assistive technology did not stop after World War I. The Second World War soon followed, again with the consequence of an increased population of individuals with disabilities (Ability Magazine, 2003; Bryant, \& Bryant, 2003; Childress, 2002; Hill, 2004; Reily, et al., 2009; United States Department of Veterans Affairs, n.d.). In the interim between wars and during World War II, significant advancements occurred not only in general technology, medical technology and pharmacology, but also in mass media. The whole nation was aware of the war and its consequences. This awareness promoted the needs of individuals with disabilities and medically-based technologies which in turn made way for the US military's provision of speech and hearing services for wounded soldiers as part of the Bardon-LaFollette Act (Bryant, \& Bryant, 
2003; Hill, 2004; Rehabilitation Act of 1943; Reily, et al., 2009). The BardonLaFollette Act provided training funds to physicians and therapists for improved methods for assisting individuals with disabilities (Bryant, \& Bryant, 2003)

During the early $20^{\text {th }}$ century technology began to move communication methods beyond manual sign use. Case in point is the invention of speech synthesis in the 1920s (Hill, 2004; Zangari, et al., 1994). Other basic technologies soon followed during the 1930 and 1940s including the transistor and some emerging technology for computers (Hill, 2004; Zangari, et al., 1994). In 1952, Geoffrey Dummer developed the integrated circuit, the basis for all modern computers. Thus, by the early 1950 s, the foundational technology for all modern AAC devices was created (Hill, 2004; Zangari, et al., 1994).

\section{Growth and Legislation}

Although the United States had endured two World Wars, it continued to find itself leading several military conflicts. More soldiers were sent to fight during the Korean and Vietnam Wars. Veterans returned to the States with disabling conditions from their time in service. Medical knowledge gained from the first World Wars progressively developed. This provided continued improvement in the survival rate not only for trauma patients, but premature births as well as strokes. With an increase in survivorship came a larger body of individuals unable to rely on verbal communication to make their thoughts and desires known (Glennen, \& DeCoste, 1997).

The precedent of the World Wars provided a foundation for the national response not only for the continuing stream of war veterans, but also for others 
experiencing disabilities. This era was also a time for expansion and growth for disability awareness and advocacy in America (Glennen, \& DeCoste, 1997). The Vocational Rehabilitation Amendments (1943, 1954 and 1965) were major additions to support individuals with disabilities and their needs for assistive technology. It "reshaped and expanded the collaboration between federal and state governments in helping people with disabilities obtain job training and find work" through the U.S. Civil Service Commission (Ability Magazine, 2003, n.p., para 8). It directed federal and state agencies to encourage and support the hiring people with disabilities (Ability Magazine, 2003).

On the larger stage, the United States was going through a national paradigm shift with regard to minority populations, including those who were disabled. The national awareness of the needs of minorities was brought to light, followed by both soft and hard policy changes (Ability Magazine, 2003; Alm \& Parnes, 1995; Hourcade, Everhart, Pilotte, West, \& Parette, 2004; Zangari et al., 1994). Soft policy changes, as described by Dr. Helander at the United Nations Development Program, were statements made by people in authority (Alm \& Parnes, 1995). They were not legally binding but could influence national views. The individuals in power during this time provided such 'soft policies' to the public conscience. For example, both President Kennedy and Vice President Lyndon B. Johnson made it known that they had family members with disabling conditions. Furthermore, throughout his presidency, Kennedy was a constant supporter of the increased awareness of the needs of individuals with disabilities 
(Hourcade, et al., 2004). A case in point was the Kennedy Panel on Mental Retardation established in 1961 (Zangari, et al., 1994).

Also at work during the 60's, the Civil Rights Movement proved to be a precedent for disability rights. The enacted civil rights legislation, the Civil Right Act of 1964 and the Civil Rights Act of 1968, did not deal directly with the needs of individuals with disabilities (Zangari, et al., 1994). However, it provided a model for advocacy, litigation and legislation for individuals with disabilities (Zangari, et al., 1994). The only legislation within this time frame that did address disability rights, the Architectural Barriers Act of 1968 (ABA) was ineffective because it was limited in scope, and had no funding or follow-up by federal agencies (Ability Magazine, 2003).

Up to this point, advocacy for the needs of the disabled came from government agencies and subsequently materialized in the form of the Rehabilitation Acts. Beginning in the 1950s the source of advocacy shifted. Individuals with disabilities and their families, especially parents, became involved in addressing the needs in both education and the workplace. In 1950, a group of parents and other invested individuals in Minneapolis Minnesota met to organize their efforts in advocating for their children (The ARC, 2011). They became The ARC, The Association for Retarded Citizens (The ARC, 2011). Their focus was for educational change, increased awareness, and keeping their loved ones out of institutions (The ARC, 2011). At that time programming and assistance for children or adults with intellectual disabilities was relatively 
unknown (The ARC, 2011). The ARC took action by funding research on intellectual and developmental disabilities (The ARC, 2011).

\section{Intervention Expansion}

Research for methods to help the disabled was greatly needed.

Professionals felt that children with disabilities developed in the same manner as normal children, only slower (Hourcade, et al., 2004). Speech and language development was viewed as a single ability, and the overall goal of intervention was verbal communication (Hourcade, et al., 2004).

From this paradigm, other methods and theories began to emerge. The efforts of parents, educators and professionals alike bore fruit. In the 1950s practitioners began to apply methods developed in the 1940 s for war veterans to individuals with cognitive and communication disabilities (Zangari, et al., 1994). The population receiving services also expanded to include not only mild impairments but those with moderate or severe disabilities (Zangari, et al., 1994). Communication boards and unaided communication (manual sign) were developed for individuals without verbal communication (Hourcade, et al., 2004; Zangari, et al., 1994). The 1950 s became the "decade for appearance of the methods and practice that is AAC" (Zangari, et al., 1994, p 29).

Also during the 40's and 50's, many limitations for the use of AAC continued to exist. Educators and interventionists believed that individuals needed to demonstrate prerequisite skills to be able to fully use these low or no tech forms of AAC (Zangari, et al., 1994). Unless the person could imitate or make sounds, comprehend or use some form of verbal language and attend to a 
task while remaining seated and using eye contact, they were not considered as a candidate for these early forms of AAC (Zangari, et al., 1994).

\section{Legislative Impact on AAC}

Within the developmental process of a professional discipline exists the refining work of disagreement. $A A C$ is no exception. This is evidenced in the disparity regarding when AAC began as a discipline. As implied before, some believe the 1950's are the starting point (Glennen, \& DeCoste, 1997; Hourcade, et al., 2004; Zangari, et al., 1994). Others, and occasionally the same individuals, take a more conservative view declaring the 1970s as AAC's inception (Bryant, \& Bryant, 2003; Lloyd, Fuller, \& Arvidson, 1997; Ogletree, \& Harn, 2001). Still others choose an even later time frame (Higginbotham, et al., 2007; McNaughton, 1990). Despite the disagreement regarding AAC inauguration, the laws developed in the 1970's provided the legal impetus that opened the doorway to services and equality for individuals with disabilities within the United States (Alm \& Parnes, 1995; Bryant, \& Bryant, 2003; Glennen, \& DeCoste, 1997; Hourcade, et al., 2004; Lloyd, et al., 1997; Ogletree, \& Harn, 2001; Zangari, et al., 1994). Specific 'hard policies' or written text for legislation and laws (Alm \& Parnes, 1995) for individuals with disabilities were first introduced in 1973 (Bryant, \& Bryant, 2003; Hill, 2004; Ogletree, \& Harn, 2001;

U.S. Department of Education Office of Civil Rights, [EDOCR, 2009]). Furthermore, these federal laws directly contributed to the growth and advancement of the field of $A A C$ as well as communication disorders and pediatrics. 
The first law to address "equal consideration and treatment of individuals with disabilities and established services and supports to gain full participation in society" was Section 504 of the Rehabilitation Act of 1973 (Bryant, \& Bryant, 2003; Hill, 2004, slide 4.14; Ogletree, \& Harn, 2001; U.S. Department of Education Office of Civil Rights, [EDOCR, 2009]). Section 504 was the initial federal law addressed the civil rights for all individuals with disabilities. It stated that

"no otherwise qualified individual with a disability in the United States ...shall, solely by reason of her or his disability, excluded from the participation in, be denied the benefits of, or be subjected to discrimination under any program or activity receiving Federal financial assistance..."(29 U.S.C.§ $749[\mathrm{~b}])$

Section 504 had broad-reaching effects for individuals with disabilities by ensuring equal access to any program receiving federal funds, such as public schools, housing, colleges, universities, or post-secondary vocational or adult education programs (EDOCR, 2009). The intent of this law and other civil rights laws was "to help deliver the promise that every individual has the right to develop his or her talents to the fullest" (Hill, 2004, slide 4.14; EDOCR, 2009).

Section 504 of the Rehabilitation Act of 1973 opened the door for other legislation to be passed, such as the Education for All Handicapped Children Act (P.L. 94-142), in 1975. P.L. 94-142 established the rights of children with disabilities between the ages of 3 and 21 to a free appropriate public education (FAPE) regardless of the degree of impairment (Bryant, \& Bryant, 2003; Hill, 2004; Hourcade, et al., 2004; Lloyd, et al., 1997; U.S. Office of Special Education Programs, 2005; Zangari, et al., 1994). Based upon this law, children were to 
receive appropriate resources and placement in the least restrictive environment (LRE) or the most normal setting possible (Bryant, \& Bryant, 2003; ED, 2000; Hill, 2004; Hourcade, et al., 2004; Lloyd, et al., 1997; Zangari, et al., 1994). These appropriate resources included the provision of speech therapy and AAC. Disability rights and services continued to gain support through new laws such as the Rehabilitation Amendments of 1984 (PL $98-221$ ), The Rehabilitation Act Amendments of 1986 (PL $99-506$ ), Education of the Handicapped Act Amendments of 1986 (PL 99 - 457), and Handicapped Chidlren's Protection Act of 1985 (PL 99 - 372) (Zangari, et al,, 1995). These laws provided support for communication services and the use of technology for individuals with severe disabilities (Zangari, et al., 1994). Unlike earlier laws, such as the $A B A$, that did not provide funds to back expectations, the current provisions did grant federal funding (Zangari, et al., 1994).

The first federal law that first specifically addressed technology was P.L. 100-407, the Technology-Related Assistance for Individuals with Disabilities Act, or Tech Act, of 1988 (ED, 2005;Glennen, \& DeCoste, 1997; Hill, 2004; Hourcade, et al., 2004 ; Lloyd, et al., 1997; Zangari, et al., 1994). The Tech Act expanded disability's sphere of influence to include the realms of assistive technology devices and assistive technology services. It also provided funding to "develop statewide, consumer-responsive information and training systems designed to meet the assistive technology needs of individuals with disabilities" (ED, 2005, p. 17). Through this funding, the Kentucky Assistive Technology System (KATS) Network was established in 1989. 
Federal laws continue to support disability rights. In 1990, the Amendments to the Rehabilitation Act of 1973 by the Americans with Disabilities Act (ADA) (P.L. 101-336) was passed. The impact of the ADA was the prohibition of discrimination based on disability (Glennen, \& DeCoste, 1997; Hill, 2004; Hourcade, et al., 2004; Lloyd, et al., 1997; Zangari, et al., 1994). It unified previous laws and extended discrimination protection into the private sector (ED, 1991; Frieden, 2005; Glennen, \& DeCoste, 1997; Hill, 2004; Hourcade, et al., 2004; Lloyd, et al., 1997; Zangari, et al., 1994).

The most recent federal law affecting individuals with disabilities is the Individuals with Disabilities Education Act (IDEA). The IDEA and the Individuals with Disabilities Education Act Amendments, 1997 and 2004, are also revisions of Section 504 of the Rehabilitation Act of 1973, as amended, 29 U.S.C. $\S 794$. This law protects the rights of students with disabilities by ensuring that everyone receives a free appropriate public education (FAPE) (Glennen, \& DeCoste, 1997; Hill, 2004; Hourcade, et al., 2004 ; Lloyd, et al., 1997; Zangari, et al., 1994).

\section{Technology Innovations in AAC}

Besides new legislation, the 1960's and 1970's saw continued developments in AAC methodology and technology (Glennen, \& DeCoste, 1997; Hill, 2004; Lloyd, et al., 1997). The Cybernetics Research Institute (CRI) developed communication methods using picture and letter selection (Hill, 2004) that led to the use of a system of switches for text generation (Glennen, \& DeCoste, 1997; Lloyd, et al., 1997). By 1969, similar technology (text-printing communication system) was developed by the Prentke Romich Company (PRC). 
By 1971, Blissymbols, a symbolic language system, was first used with nonverbal children at the Ontario Crippled Children's Center (Hill, 2004; Glennen, \& DeCoste, 1997; Lloyd, et al., 1997). The first programmable microprocessorbased AAC system soon followed in 1972 (Hill, 2004; Zangari et al., 1994). Then in 1978, AAC devices produced the first synthetic speech, thereby creating the first voice output communication device (VOCA) (Hill, 2004; Glennen, \& DeCoste, 1997; Lloyd, et al., 1997; Zangari, et al., 1994).

Although much of the evidence for the use of AAC up to this time was anecdotal (Lloyd, et al., 1997), AAC began to be viewed as a legitimate form of communication by the end of the 1970s (Bryant, \& Bryant, 2003; Lloyd, et al., 1997; Ogletree, \& Harn, 2001). Soon systematic research of this discipline began, and with it the myth that AAC would diminish verbal communication was dismissed (Glennen, \& DeCoste, 1997; Lloyd, et al., 1997; Zangari, et al., 1994). The 1970s saw an increase in AAC means or methods. Communication methods increased to an array of traditional orthographic symbols, tangible symbols, facilitated communication, lesograms, and manual signs (Ogletree, \& Harn, 2001).

The increase in the availability of communication devices and methods brought about other needed changes. One such development was the standardization of technology design (Zangari, et al., 1994). Growth was also evident in the beginning of discussions regarding use of AAC for specific populations (Zangari, et al., 1994). One particular population, individuals with 
autism spectrum disorders, had their first introduction to AAC (Ogletree, \& Harn, 2001; Zangari, et al., 1994).

\section{Development of AAC as a Profession}

In the 1980 s and into the 1990 s, AAC expanded as a profession and refined as a specialization. Although AAC is considered within the realm of communication disorders (ASHA, 1991), as mentioned previously, it is also part of assistive technology. Due to this overlap, AAC is a multidisciplinary field (Glennen, \& DeCoste, 1997). Other professionals who may be involved include occupational therapists, special educators, and rehabilitation engineers (Glennen, \& DeCoste, 1997). Vendors have also played a significant and active role within the field, which is unusual (Zangari, et al., 1994). Manufactures may provide consultation services, training, as well as advocacy (Zangari, et al., 1994).

In 1981, ASHA released a position statement regarding AAC (Zangari, et al., 1994; ASHA, 1981). This document, written by the Ad Hoc Committee on Communication Processes and Nonspeaking Persons, became an official policy statement in 1980 (ASHA, 1981). It provided validation to the new field by defining its terminology, reviewing the history of its development, defining its service-delivery model, reviewing professional preparation and professional ethics (ASHA, 1981). The International Society for Augmentative and Alternative Communication (ISAAC), and the first doctoral program were established soon after, in 1983 (Zangari, et al., 1994). 
The discipline of AAC grew quickly due to not only from laws, but also due to provider's improved knowledge of disabilities and technological resources. The 1980 s and early 90 s also saw significant transformations in intervention methodologies. The focus of intervention moved from the candidate model to the communication needs model (Glennen, \& DeCoste, 1997; Hourcade, et al., 2004). Within this model, the goal was simply to identify an individual's unmet communication needs and then fulfill that need (Glennen, \& DeCoste, 1997; Hourcade, et al., 2004). The communication needs model was a reflection of other intervention developments of that time. This can be seen in its consideration of oral-motor abilities, multiple modes of communication and natural context for communication (Glennen, \& DeCoste, 1997; Hourcade, et al., 2004).

Intervention methods were further influenced by changes in sources of data used for decision making. Information for decision making shifted from anecdotal to empirical evidence (Hourcade, et al., 2004). Research began to form the basis for intervention decision making (Hourcade, et al., 2004). Findings from this research shaped professional decision making regarding communication models, communication competency, assessment, as well as intervention and service delivery (Hourcade, et al., 2004). These findings began to be disseminated through books and literature specifically about AAC (Hourcade, et al., 2004).

From this information, providers better understood how cognitive and social development delays affected the development of communication skills 
(Hourcade, et al., 2004). They found that individuals with cognitive or social development delays did not follow the typical path for communication development (Hourcade, et al., 2004). Interventionists then began to look beyond the typical developmental path for communication (Chapman \& Miller, 1980). This shifted intervention strategies from a focus on grammatical development to the use of language as a social behavior, or functional communication skills (Hourcade, et al., 2004). Thus, communication training began the "use of naturally occurring opportunities to teach communication during the course of an individual's daily routines." (Hourcade, et al., 2004, p, 239). Naturalistic and functional training was found to improve communication development as well as the generalization of these skills (Hourcade, et al., 2004).

Besides instruction for functional communication, another shift in thinking was the use of more than one communication system by an AAC user (Hourcade, et al., 2004). In 1988, Musslewhite, suggested combining systems for the best communication outcome (Hourcade, et al., 2004). This simple suggestion opened up many options for users. Fortunately, methodological and technological developments were providing more communication choices than ever before. Those options included sign language, gestures, and a variety of picture symbols, symbol systems (e.g. Rebus, Blissymbols; Non-Speech Language Acquisition Program) and early electronic communication devices (e.g. scanning devices, simple switches and eye-gaze boards) (Hourcade, et al., 2004). Computer technology also provided a selection of AAC devices, or voice output communication devices, using speech synthesis (Hourcade, et al., 2004). 
AAC devices and options had also become smaller, more affordable, and more commercially available (Hourcade, et al., 2004).

\section{Current Trends}

Today by definition AAC is an area of research, clinical and educational practice (ASHA, 2004). AAC practitioners are to "study and when necessary compensate for temporary or permanent impairments, activity limitations, and participation restrictions of persons with severe disorders of speech-language production and or comprehension, including spoke and written modes of communication" (ASHA, 2004, pg. 1). Consideration of AAC not only includes a method, but also a system of communication. By definition, AAC is a method whereby individuals use "linguistic rules by which symbols are selected and combined to transmit the various forms, contents and uses of language" (ASHA, 2004, p. 1). At the same time, AAC is also system composed of any number of 'symbols, aids, strategies and techniques' to support meaningful and active communication" (ASHA, 2004, p. 1). This system or array of communication means is not static to the user, but goes through an ongoing process of change to meet the user's needs and taste (ASHA, 2004). The end goal for AAC use must always be to allow individuals to independently communicate whatever they want wherever they want as fast as they can (ASHA, 2004; Hill, 2004).

As with the use of any tool, a level of system competency needs to be achieved by the AAC user. Light, Beukleman and Reichle (2003) described four different competencies the AAC user needs to demonstrate: linguistic, operational, social and strategic. Not only must an AAC user show skill within language use 
(linguistic competency), but also how to apply that language across a variety of social situations (social competency) or when communication breakdowns occur (strategic competency) (Light, et al., 2003; Sigafoos, et al., 2011). Beyond language, the user must also show a level of ability in basic operations of their system (operational competency) (Light, Beukleman \& Reichle, 2003; Sigafoos, et al., 2011).

AAC systems are now identified within a range of technology. A leader in the study of AAC systems, the University of Buffalo, defined the each level within the range (Hill, 2004). No tech systems are ones that do not need a power source (Hill, 2004). Low tech or light tech systems require a power source and are easy to use (Hill, 2004). Mid tech systems require a power source and require some training to program and maintain the device (Hill, 2004). High tech systems require a power source and extensive training to program and maintain the device (Hill, 2004). The cost of the respective systems typically increases as they move up the levels of technology. According to the 2010-2011 price lists, some of the high tech devices can cost more than eight-thousand US dollars (i.e., V and VMax, [Dynavox, 2011]).

Although the details regarding AAC systems and methods are worthy of study, the most important variable within the equation is the user. The person in need of, or using AAC, is considered the main stakeholder. Stakeholders are individuals with invested interest in the AAC service delivery process. The service delivery model for AAC should therefore be consumer-centered with the focus on the needs of the AAC user guiding all decisions (Blackstone, Williams \& 
Joyce, 2002; Buekelman \& Mirenda, 2007; Calculator \& Black, 2009; Hill, 1998). Some discussion has risen regarding what to call an AAC user. International Society for Augmentative and Alternative Communication (ISAAC) recommends the use of term complex communication needs $(\mathrm{CCN})$ when discussing people 'who rely on AAC' (Hill, 2004). Some see this term as difficult to use when identifying individuals for research (Alant, Bornman, \& Lloyd, 2006) because the label does not identify the degree of disability, and is imprecise (Alant, et al., 2006).

No matter their label, individuals who use or could benefit from AAC come from all socioeconomic, ethnic, and racial backgrounds (ASHA, 1993; Buekelman \& Mirenda, 2007; Hill, 2004). These individuals may range in age from infant/toddlers to geriatrics, and may be diagnosed with a variety of disabilities (ASHA, 1993; Buekelman \& Mirenda, 2007; Hill, 2004). Many conditions are associated with the need to use AAC. Some people experience congenital (from birth) conditions, such as cerebral palsy, autism spectrum disorders, mental retardation or Down syndrome (ASHA, 1993; Buekelman \& Mirenda, 2007; Hill, 2004). Other children have acquired conditions that involve the loss of their ability to speak. Traumatic brain injury, muscular dystrophy or other motor neuron diseases are examples of acquired disabilities that may disable a previously intact speech and language system (ASHA, 1993; Buekelman \& Mirenda, 2007; Hill, 2004).

The need for AAC intervention depends on the severity or progression of the disorder. For example, the AAC system may only be needed for 
rehabilitation (Buekelman \& Mirenda, 2007; Hill, 2004). However, AAC may need to be used across all communication environments as a motor neuron disease (e.g. ALS) takes its course and progressively limits the person's ability to verbally communicate (Buekelman \& Mirenda, 2007; Hill, 2004). The common thread between these individuals is an inability to use speech for functional, independent communication (ASHA, 1993; Buekelman \& Mirenda, 2007; Hill, 2004). The important decision point is inadequate speech to meet all communication needs (ASHA, 1993, Hill, 2004; Buekelman \& Mirenda, 2007). Any level of need may qualify within the zero exclusion criterion (ASHA, 2004).

\section{Communication Models}

The concept of zero exclusion fits with the current model for assessment and intervention, the Participation Model. Previously, AAC users had to demonstrate eligibility for an AAC system (Candidacy Model) or help figure out which device met their communication need (Communication Needs Model) (Buekelman \& Mirenda, 2007; Hourcade, et al., 2004). The Participation Model operates on the belief that all people can communicate (Hourcade, et al., 2004). The instead of device selection, the main issues to be addressed are communication opportunities and communication access (Hourcade, et al., 2004). Providers are directed to look at the communication patterns of the AAC user, their communication needs throughout the day and then identify communication opportunities and access barriers to those opportunities (Buekelman \& Mirenda, 2007; Hourcade, et al., 2004). An AAC system(s) is/ are selected based on the overall communication needs of the user. 


\section{AAC Technology Issues}

Standardization and evidence-based practices are a continuing issue. There continues to be a lack of standardized, evidence-based procedures for identifying whether or not an individual would benefit from AAC (Alant, et al., 2006; ASHA, 2004). A current battery of assessments for AAC is also needed (Alant, et al., 2006; ASHA, 2004).

Technology for communication systems has advanced considerably (e.g. IPAD applications) but is still lacking some critical components. For example, in view of the need for face-to-face interaction during typical communication, an AAC user finds himself at a disadvantage. He has to divide his attention between his system and communication partner (Alm \& Parnes, 1995). The time needed to retrieve messages from a system for rapid interaction also currently limits spontaneous communication (Alm \& Parnes, 1995). New means for input, access and retrieval need to be developed to close the gap between verbal and AAC communication means. One possible solution would be for technology to 'learn' a users communication pattern and individualize it for improved ease of use (Alm \& Parnes, 1995).

Another missing component for AAC is embodied in its synthesized voice output. Synthesized voices do not provide prosodic flexibility needed for emotional expression. Research is addressing this by attempting to embed emotion within synthetic speech (Alm \& Parnes, 1995). 


\section{ACC Implementation Barriers}

Perhaps the greatest need for improvement is the care of young users who are largely overlooked. Many children are not referred for AAC services until they are older (if then) and miss out on learning opportunities during crucial developmental periods (Light, \& Drager, 2007). Referrals for services are not made even though it is "never too early to incorporate AAC into language/ communication intervention for young children with significant communication disabilities" (Light, \& Drager, 2007, p. 212).

The consequences for a lack of early intervention for AAC services can be significant. A lack of an appropriate communication system can hinder a child's overall communication development (Light, \& Drager, 2007). Constraints on a child's vocabulary, or symbol selection, are an 'artificial constraint' on their ability to communicate (Light, \& Drager, 2007, p. 212). The lack of a communication system can exacerbate preexisting communication delays and may hinder future literacy development, academic development, and social development (ASHA, 2007). Deficient communication means may also cause behavior problems. When a child's ability to communication is limited or nonexistent, they will use their current means for expression, which may include negative behavioral responses or interaction (Downing \& Siegel-Causey, 1988).

Many children that require $A A C$ are often not referred until they are well beyond preschool years (Light, \& Drager, 2007). Reasons for the lag in referrals include negative attitudes, decreased expectations for a communication system, 
a lack of advocacy, a lack of knowledge and diminished or limited funding resources (Light, \& Drager, 2007).

These barriers call for changes within the service provision for young children. This population needs increased advocacy by healthcare professionals (Light, \& Drager, 2007). Advocacy can come through a better understanding of AAC through professional education, training and in-services. This is in turn can lead to better early identification and early intervention for young children with complex communication needs (Light, \& Drager, 2007). The quicker children are served the fewer opportunity barriers they will experience (Light, \& Drager, 2007).

\section{Historical Chronology of Graduate Medical Training}

The study of medicine dates from mankind's distant past. In comparison, the study of pediatrics and the implementation of medical residency within medical education programs are recent phenomena. Formalized educational standards by the American Medical Association (AMA) made way for the release of the historic report, Medical Education in the United States and Canada, by Abraham Flexner (University of Louisville, 2008). Flexner's 1910 report impacted medical education throughout the United States by improving standards for curriculum, admission and graduation (American Medical Association, 2008; University of Louisville, 2008). With the improvement of educational standards, it soon became clear that there was a need for standardization within the hospital internship programs (American Medical Association, 2008). This 
acknowledgement was the final step toward the inception of residency programs in the United States (American Medical Association, 2008).

In defining the resident population, the American Medical Association (AMA) identifies residency as "the period of training in a specific medical specialty" (American Medical Association, 2008, [Data File] para, 8). Medical residency occurs after a student completes four years of undergraduate and premedicine training and then graduates from four years of medical school (American Medical Association, 2008). Through a national matching program, newly graduated medical doctors enter into a residency program that is three-toseven years of professional training under the supervision of senior physician educators (American Medical Association, 2008).

The desired outcome for resident education is a competent pediatrician. By definition pediatricians practice the "specialty of medical science concerned with the physical, emotional, and social health of children from birth to young adulthood" (Goodman, 2005, p. 56). The responsibilities of the pediatrician encompass a broad spectrum of health services ranging from preventive health care to the diagnosis and treatment of acute and chronic diseases (American Academy of Pediatrics, 2008; Goodman, 2005). These responsibilities include understanding what factors affect a child's growth within both their physical and mental development (American Academy of Pediatrics, 2008; Goodman, 2005). Due to a child's dependency on the home and family, one of these factors is a nurturing home environment. Pediatricians are to educate and guide families to live healthy, to participate in community services, to prevent or solve problems in 
health care, and to advocate for the needs of children (American Academy of Pediatrics, 2008; Goodman, 2005).

\section{Accreditation of Graduate Medical Programs}

Since 1927, medical residency programs in the United States have continued to thrive. In looking at the Accreditation Council for Graduate Medical Education's (ACGME) 2008 - 2009 data resource book, there were 109,482 residents and fellows on duty at 688 sponsoring institutions in the United States (American Council for Graduate Medical Education, 2009 - 2010). Onehundred-and-ninety-four of those institutions housed pediatric programs with 8,874 total residents (American Council for Graduate Medical Education, 2009 2010). The University of Louisville's School of Medicine is among the institutions accredited to provide medical education (American Academy of Pediatrics, 2008).

In looking further at program accreditation for general medical education, there are a number of organizations governing its provision. The ACGME is one of the most recognized. It was established in 1981 from a consensus in the academic medical community to provide an independent accrediting organization (ACGME, 2000). The Accreditation Council for Graduate Medical Education's (ACGME) member organizations include the American Board of Medical Specialties, American Hospital Association, American Medical Association, Association of American Medical Colleges, and the Council of Medical Specialty Societies (ACGME, 2000). 
The forerunner to the ACGME was the Liaison Committee for Graduate Medical Education (LCME), established in 1972 (ACGME, 2000). This organization continues to provide additional accreditation for medical education programs in both the United States and Canada (ACGME, 2000). Most state boards of licensure require that U.S. medical schools are accredited by the LCME, as a condition for licensure of their graduates (ACGME, 2000). The University of Louisville is currently accredited by the LCME through 2013 (Liaison Committee on Medical Education, 2010).

Another accrediting body is the Association of American Medical Colleges (AAMC). This organization accredits medical schools and teaching hospitals in Canada and the United States, but is known more as the administrator of the Medical College Admission Test, also known as the MCAT (Association of American Medical Colleges, 2010). The AAMC also operates the American Medical College Application Service (AMCAS) and the Electronic Residency Application Service (ERAS) which facilitates students applying to medical schools and residency programs (Association of American Medical Colleges, 2010).

Beyond accreditation, several governing bodies provide guidance and regulation of resident training. In specifically looking at pediatrics, these organizations include the Ambulatory Pediatric Association (APA), the American Academy of Pediatrics (AAP), and the ACGME (ACGME, 2010; ACGME, 2007; American Medical Association, 2008). Beginning with the Ambulatory Pediatric Association (APA), it provides educational guidelines related to residency training 
in community pediatrics settings (Ambulatory Pediatric Association, 2010). The American Academy of Pediatrics (AAP) provides rules for pediatric resident training, but this is done through several subsidiaries (American Academy of Pediatrics, 2008). These include the Future of Pediatric Education II (FOPE II) and the AAP Council on Medical Student Education in Pediatrics (American Academy of Pediatrics, 2008). The final agency is the ACGME. Within the ACGME, The Pediatrics Residency Review Committee (RRC) establishes the standards and accreditation criteria for pediatric training in the United States (American Academy of Pediatrics, 2008).

All of the previously mentioned entities provide specific pathways that must be followed for the completion of medical training. The milestones consist of mandatory medical examinations medical students and residents must pass. The primary series of evaluations is the three steps of the United States Medical Licensing Examination (USMLE) (United States Medical Licensing Examination, 2010). Medical students take a three-part exam during medical school and residency (United States Medical Licensing Examination, 2010). After passing all three assessments they are eligible to apply for their medical license and state board certification to practice as a physician (United States Medical Licensing Examination, 2010). The first two steps of the USMLE are taken during medical school, followed by step three which is taken during the first or second year of residency (United States Medical Licensing Examination, 2010). During residency, the USMLE also provides annual 'in-training' examinations to assess 
an individual resident's readiness for the board examination and to track educational progress (United States Medical Licensing Examination, 2010).

The final step to recognition as a certified pediatrician is passing the board examinations (American Medical Association, 2008). In order to take the board examinations, a resident must graduate from an accredited medical school in the U.S. recognized by the World Health Organization (WHO) after completing three years of training in pediatrics in an accredited residency program. Residents must also present satisfactory completion of residency training, get a valid unrestricted state license to practice medicine, and pass the two day written exam for board certification (American Medical Association, 2008). During 2003 there was a $78 \%$ certification rate for pediatricians nationally (Association of American Medical Colleges, 2010). Board-certified pediatricians are members of the American Academy of Pediatrics (American Academy of Pediatrics, 2008).

The pediatrics residency program within the Department of Medicine at the University of Louisville has always reflected the evolution of medicine within American history. The establishment of a medical college in Louisville began in 1833 with a simple committee meeting. The results of that meeting were the first medical classes at $U$ of $L$ in 1837 (University of Louisville, 2008). At that time the medical training did not have specific guidelines to follow. It wasn't until 1847 that the American Medical Association (AMA) began a Committee on Medical Education (University of Louisville, 2008). Over fifty years later, in 1904, the Council on Medical Education was finally formed by the AMA to address 
education standards for physicians within the United States (American Medical Association, 2008).

In Kentucky, there are four ACGME accredited institutes with a total of 99 programs and 1,119 residents (Association of American Medical Colleges, 2010). The University of Louisville is one of the four ACGME accredited institutions. The University of Louisville's School of Medicine's educational program was recently reaccredited by the ACGME in 2009. The University of Louisville sponsors the most residency programs (52), and it has the most residents, (569)(Association of American Medical Colleges, 2010) in Kentucky. Of the 569 residents, 102 are currently on duty within the pediatrics program (Association of American Medical Colleges, 2010). The basic pediatric residency program at the University of Louisville is three years long (Association of American Medical Colleges, 2010).

\section{Current Pediatric Practices}

The journey to receiving services for a communication disorder and AAC often begins with a parent bringing their child and their concerns to their pediatrician's office. As previously noted, pediatricians play a substantial role in the ongoing care of children, but this is especially true for children with disabilities. Within the medical community, children demonstrating a disability, such as a communication disorder or a need for AAC, are categorized as children with special health care needs (CSHCN) (McPherson, et al., 1998; Sadof, \& Nazarian, 2007; Ziring, et al., 1999). As defined by the Maternal and Child Health Bureau and adopted by the American Academy of Pediatrics (AAP), 
CSHCN have or are at "increased risk for a chronic physical, developmental, behavioral, or emotional conditions and require health and related services beyond" what is normal (Ziring, et al., 1999, p.978). These children represent $13 \%$ of the total pediatric population but $70 \%$ of all pediatric health care expenditures (Ziring, et al., 1999).

With the substantial care and cost expenditure these children represent, a number of policies have been developed to not only protect this population but also ensure an appropriate standard of care (Ziring, et al., 1999). Current professional practice policies have transformed how today's pediatricians address these needs (ACGME, 2007; American Academy of Pediatrics, 2002; American Academy of Pediatrics, 2005; American Academy of Pediatrics Advisory Committee, 2002; Stille, \& Antonelli, 2004; Ziring, et al., 1999). These policies include the concepts of the medical home, care coordination, licensure for developmental-behavioral pediatrics and the specification of educational competencies by the ACGME.

\section{Medical home}

To meet the complex and costly needs of CSHCN the US Department of Health and Human Services' Healthy People 2010 goals stated that "all children with special health care needs will receive regular, ongoing, and comprehensive care within a medical home" (Ziring, et al., 1999, p. 980). This policy is reflected in the educational standards for pediatric residents (ACGME, 2007; ACGME, 2007). As stated in the Future of Pediatric Education II goals and objectives, 
"pediatric medical education at all levels must be based on the health needs of children in the context of the family and community" (Ziring, et al., 1999, p. 981).

In looking at the history behind the term, the concept of the medical home is not new. The phrase first appeared in 1967 in a book by the AAP's Council on Pediatric Practice (Ziring, et al., 1999; Sia, Tonniges, Osterhus, \& Taba, 2004). At that time the goal was the centralization of records for CSHCN (Ziring, et al., 1999; Sia, et al., 2004). The centralization of records was thought to support improved health care and health care supervision (Sia, et al., 2004). By 1974, the AAP concept became policy, and the focus also included decreasing "costly, scattered and less efficient services" (Sia, et al., 2004, p. 1475).

Today, with increasing health care costs, technology, survivorship, medical specialization, and fragmentation of care, the concept of the medical home is gaining interest and standing. It is moving beyond the realm of medicine and into the public sector (American Academy of Pediatrics Advisory Committee, 2002; American Academy of Pediatrics, 2002; Sia, et al., 2004). Today the medical home is assumed to be normal protocol for the treatment of all CSHCN. The term now encompasses a "partnership approach with families to provide primary health care that is accessible, family centered, coordinated, comprehensive, continuous, compassionate and culturally effective" (Sia, et al., 2004, p. 1473). With this definition in mind, whenever medical care is provided, a physician must ensure that a specific level of care is met.

These standards of care for pediatric populations within the medical home are outlined by the AAP (American Academy of Pediatrics Committee on 
Community Health Services, 1999; American Academy of Pediatrics, 2002; American Academy of Pediatrics Advisory Committee, 2002). The first standard is to be a family-centered provider that develops a 'trusting partnership' with those in your care (American Academy of Pediatrics Committee on Community Health Services, 1999; American Academy of Pediatrics, 2002; American Academy of Pediatrics Advisory Committee, 2002). As part of this partnership, the pediatrician must identify the needs of child and family and refer the CSHCN to the appropriate services (American Academy of Pediatrics Committee on Community Health Services, 1999; American Academy of Pediatrics, 2002; American Academy of Pediatrics Advisory Committee, 2002). Trust is established through the presentation of information in a clear and unbiased manner as well as through continuity of care with transition services when the child grows into adulthood (American Academy of Pediatrics Committee on Community Health Services, 1999; American Academy of Pediatrics, 2002; American Academy of Pediatrics Advisory Committee, 2002). Another expectation for pediatricians is to be knowledgeable about specialty and community services or organizations that are available and accessible (American Academy of Pediatrics, 2002; American Academy of Pediatrics Advisory Committee, 2002). Their knowledge should be based on communication with early intervention programs, schools, early childhood education programs or other necessary agencies that address the need of the child and family (American Academy of Pediatrics, 2002; American Academy of Pediatrics Advisory Committee, 2002). 
As can be seen from the current standards, the original model of the medical home, (i.e., housing complete central records about the child), continues to be one aspect of the policy, but only a very small part (Sia, et al., 2004). The emphasis today is the comprehensive coordination of care within the context of individualized, family-based planning (Ziring, et al., 1999). When the goals of the medical home are achieved, the CSHCN, or specifically a child with a communication disorder is sure to benefit from a better quality of service.

The objective and AAP policy of the medical home has yet to be completely embraced by the pediatric medical system (American Academy of Pediatrics Advisory Committee, 2002; American Academy of Pediatrics, 2002). Medical system complexity and uncoordinated care were found to be the major barriers between the current state of pediatric health care and an effective health care system for CSHCN (American Academy of Pediatrics Advisory Committee, 2002; American Academy of Pediatrics, 2002).

\section{Care coordination}

The provision of a medical home alone is a great responsibility to be shouldered, but for today's pediatricians there are even more professional expectations to be met. The coordination of care is one of those expectations (Ziring, et al., 1999). Care coordination involves the family, the physician, and other professionals working together to implement a specific care plan for an individual child as an organized team (Ziring, et al., 1998). It not only links children and their families with appropriate services and resources, but ensure 
quality services (American Academy of Pediatrics Advisory Committee, 2002; American Academy of Pediatrics, 2002).

The policy for care coordination overlaps that of the medical home in its emphasis on the provision of quality services for children. The two policies also dove-tail or complement each other since the best environment for care coordination to occur is within the continuity of the medical home (American Academy of Pediatrics Advisory Committee, 2002; American Academy of Pediatrics, 2002).

Care coordination involves a process. The process includes an assessment or identification of needs, creation of a plan of care, implementation of the plan, and follow-up evaluation of outcomes (American Academy of Pediatrics Advisory Committee, 2002; American Academy of Pediatrics, 2002). Within the care coordination process, the pediatrician acts as the practice care coordinator and organizes, organizes and collaborates with other providers, agencies, and organizations involved with the care of the patient (American Academy of Pediatrics, 2005; Stille, \& Antonelli, 2004; Ziring, et al.; 1999). The pediatrician is placed in this role under the AAP's authority. Within AAP's policy it states that the pediatrician is "uniquely suited to manage, coordinate, and supervise the entire spectrum of pediatric care, from diagnosis through all stages of treatment, in all practice settings" (American Academy of Pediatrics, 2005, p. 1238). The AAP recognizes the valuable contributions of non-physician professionals, but has stated that the best pediatric care is provided by using a team-based approach with a physician, preferably a pediatrician, as a leader 
(American Academy of Pediatrics, 2005; Stille, \& Antonelli, 2004; Ziring, et al.1999).

In their role as the care coordinators, the pediatrician is to communicate, network, and educate as well as advocate for resources (American Academy of Pediatrics, 2005; Stille, \& Antonelli, 2004; Ziring, et al.; 1999). As the head, the pediatrician then disseminates the needed information and provides a specific reason for referrals to appropriate specialists, mental health professionals or developmental professionals (American Academy of Pediatrics, 2005; Stille, \& Antonelli, 2004; Ziring, et al.; 1999). Initiatives from health care reform and managed care have likewise expanded the role of the primary care physician as gatekeeper and coordinator of patient services (American Academy of Pediatrics Committee on Children with Disabilities, 1998).

Currently, the emphasis on care coordination is not due to cost and health management alone. It is also emphasized because of the increasing number of children with special health care needs, the increasing complexity of care, and the increased need for outreach efforts to educate about the medical home (American Academy of Pediatrics Committee on Children with Disabilities; 1998).

The benefits of care coordination are significant. It provides improved care for the immediate family and practitioner. Studies have also shown care coordination positively impacting funding and medical system use (American Academy of Pediatrics Committee on Children with Disabilities; 1998). These benefits include reduced hospital admissions, reduced length of hospital stay, reduced in patient charges, reduced emergency department visits, improved 
patient satisfaction, and enhanced opportunities for outcome-based clinical process improvement (Stille, \& Antonelli, 2004; Ziring, et al.; 1999; American Academy of Pediatrics, 2005). Successful care coordination results in optimal outcomes for CSHCN and their families and provides an opportunity for professional fulfillment for physicians (American Academy of Pediatrics, 2005; Stille, \& Antonelli, 2004; Ziring, et al.; 1999).

\section{Barriers to Service Delivery}

As with the medical home, policy and positive outcomes do not equal compliance. Numerous barriers for care coordination exist. These barriers broadly include gaps in available resources, team dynamics, medical systems management and education (Antonelli, \& Antonelli, 2004; Antonelli, Stille, \& Antonelli, 2008).

In identifying specific problems of care coordination, issues with team dynamics are easy to recognize. Communication breakdown among the individuals and organizations involved can easily affect the provision of quality care (Antonelli, \& Antonelli, 2004; Antonelli, et al., 2008). Communication can also affect role definitions and team collaboration.

Other barriers for care coordination are inherently found in the medical system. For example, service funding by payer sources requires identification of specific and complex criteria (Antonelli, \& Antonelli, 2004; Antonelli, et al., 2008; McPherson et al., 2004). The significant amount of time and effort needed for pediatricians to provide care coordination is not recognized or reimbursed by these funding sources (Antonelli, \& Antonelli, 2004; Antonelli, et al., 2008; 
McPherson et al., 2004). Therefore, families may struggle to access needed services due to the language, economic and socio-cultural barriers.

Furthermore, the family or pediatrician must also identify complex eligibility criteria (i.e., family income and the child's age and/or health condition) to determine the availability of funding and services. These criteria often differ

among organizations and agencies (Antonelli, \& Antonelli, 2004; Antonelli, et al., 2008; Gupta, O'Connor, \& Quezada-Gomez, 2004; McPherson et al., 2004). Beyond these problems, there is a lack of single point of entry into the medical system (McPherson et al., 2004). Families must initiate with and navigate through multiple specialists, offices and organizations (McPherson et al., 2004).

Barriers create an absence of care coordination. This absence results in incomplete coordination and episodic, expensive, fragmented care. These barriers in the coordination process may reflect a lack of medical student and resident training for care coordination skills (Antonelli, \& Antonelli, 2004; Antonelli, et al., 2008; McPherson et al., 2004). The complexity and number of medical liability issues demonstrate the need for pediatricians, as advocates for their patients, to educate communities, legislators and health policy makers about the necessity of the medical home and care coordination (Antonelli, \& Antonelli, 2004; Antonelli, et al., 2008; McPherson et al., 2004).

\section{Standards for Hospitals and Skilled Nursing Facilities}

Beyond the challenges of providing a medical home and care coordination, additional standards for pediatricians within hospitals and skilled nursing facilities are now in effect. These involve changes in accreditation 
regulations from the Centers for Medicare and Medicaid Services (CMS) and the Joint Commission. The new regulations both focus on the improvement of effective communication between professionals and patients (Joint Commission, 2010; Pressman, \& Blackstone, 2010). Specifically, within the CMS regulations staff and physicians are to include information within the Minimum Data Set (MDS) 3.0 that identifies individuals that have pre-existing communication impairments or adaptations, have a different primary language, or who have a diminished ability to communicate due to illness or treatment (Joint Commission, 2010; Pressman \& Blackstone, 2010). The MDS administrator has to determine if the patient's speech is intelligible or provide a means for effective communication before the Brief Interview for Mental Status and the Personal Preferences section of the assessment are completed (Joint Commission, 2010; Pressman, \& Blackstone, 2010).

If the patient is not able to be understood, they must be provided with an alternative means of communication. According to the CMS patients should be offered other communication means including but not limited to "writing, pointing or using cue cards" (Pressman, \& Blackstone, 2010, p. 7). The regulation suggests that skilled nursing facilities (SNF) staff has a "broad range of augmentative and alternative communication strategies and tools and other assistive technologies" at their disposal to assist with effective communication (Pressman, \& Blackstone, 2010, p. 7)

The new Joint Commission standard for Advancing Effective Communication, Cultural Competence and Patient - and Family-Centered Care 
went into effect in 2010. The Commission published a Road Map (Joint Commission, 2010) that provides guidance and suggestions on how to implement this standard. Within the Road Map, the Commission addresses how to improve impaired communication due to illness and medical treatment (Joint Commission, 2010; Pressman, \& Blackstone, 2010).

Adherence to the new standards is important for several reasons. A lack of communication or a breakdown of communication between patients and professionals can lead to "sentinel events, breaches of safety and reduced quality of care" (Pressman, \& Blackstone, 2010, p. 8). The other incentive for adherence is continued accreditation and operation for hospitals and skills nursing facilities.

\section{Clinical AAC report}

In further investigation of current pediatric practices, in an initiative within the American Academy of Pediatrics was released July of 2008. It was a clinical report regarding the prescription of assistive technology systems with a focus on children with communication disorders (Desch, et al., 2008). This clinical report added more standards of treatment for CSHCN to those already established through the medical home and coordinated care. It focused specifically on the pediatric population needing augmentative and alternative communication (Desch, et al., 2008).

The report emphasized the responsibility and need for knowledge regarding AAC (Desch, et al., 2008). Desch and his colleagues stated that for the 5 million children that have some type of disabling condition ( $15 \%$ of the total 
population), greater than $20 \%$ have a communication disorder (Desch, et al., 2008). The population needing AAC was quoted as ranging from a little over two to four and a half percent of the pediatric population (Desch, et al., 2008). Furthermore, the report indicated that $25 \%$ of the need for AAC was unmet (Desch, et al., 2008). As in other research (Scherer, 1990), it was found that $75 \%$ of devices are abandoned within the first year of their acquisition (Desch, et al., 2008).

The report further emphasized the need for action by pediatricians regarding AAC by reviewing the role they are to play as a medical home and care coordinator. As part of the medical home, the primary care pediatrician plays an important role in the interdisciplinary effort to provide appropriate assistive technology for communication disorders (Desch, et al., 2008). Within the paradigm of care coordination, the pediatrician is charged to recognize communication disorders in the children under their care and make appropriate referrals. The "pediatrician providing the medical home should develop a carecoordination process that involves all available resources to help families through the often complicated process" (Desch, et al., 2008 p. 1275). The complexity of the process to acquire and use a communication device is one of the same barriers that occur in care coordination. Thus, the report directly addressed some of the issues and responsibilities surrounding the role of the pediatrician and AAC (Desch, et al., 2008).

Pediatricians are not expected to know everything about new technology, but they should know enough to be an informed advocate (Desch, et al., 2008). 
The reason they need to have at least a basic understanding of AAC is because caregivers, patients or other professionals may request their referrals, opinions, prescriptions or letter of medical necessity for a device (Desch, et al., 2008). The letter of medical necessity can only be written after the pediatrician confers with members of the team who have evaluated the child (Desch, et al., 2008). It should state that the physician received the evaluation reports, reviewed the recommendations and concurred that that the recommended devices are medically necessary for treatment of the child's communication impairment caused by the specific diagnosis (Desch, et al., 2008). It may include the child's current status, expected outcome of the device as well as a report of the physical examination or diagnoses (Desch, et al., 2008).

All pediatricians, including sub-specialties who are vital to medical home, are to work together to improve access to AAC devices and programs (Desch, et al., 2008). Besides having a basic knowledge of AAC, pediatricians must have knowledge of the professionals or community resources for both communication disorders and AAC (Desch, et al., 2008). The next step is to then act as a part of the team. This involves cooperation with the diagnostic assessment process, advocacy, and short-term and long-term planning with the appropriate professionals (Desch, et al., 2008).

The clinical report is specific in defining the role of the pediatrician regarding the writing letters of medical necessity. Pediatricians are then to assist with the implementation of any of the parts of the plan. This support may include helping to find and advocate for funding sources, device procurement, device 
training, monitoring device use and therapy programs (Desch, et al., 2008). The pediatrician must help to coordinate all therapies and programs that the child is receiving with the family's needs (Desch, et al., 2008).

The final step in the process is to work closely with the family and the team of professionals (mainly education and speech-language pathology) to evaluation the effectiveness of the efforts being made and to ensure appropriate follow-up (Desch, et al., 2008). Researchers (Desch, et al., 2008) emphasized that the pediatrician may be the professional who is best able to evaluate the child's progress in relationship to the family's satisfaction or dissatisfaction. Furthermore, ongoing family satisfaction is one key to limit device abandonment.

\section{Pediatric Resident Competencies}

The extensive responsibilities of pediatricians require extensive training to fulfill their roles in the lives of children. This is the job of medical education, and specifically the goal of resident education. To ensure the best educational outcomes for today's professionals, the Accreditation Council for Graduate Medical Education (ACGME) and the American Board of Medical Specialties (ABMS) jointly developed a long-term initiative, the Outcome Project (ACGME, 2006). The ACGME Outcome Project developed two goals: 1) to make sure residency program content meets the changing needs of today's health care system, and 2) to establish valid outcome assessment systems to measure a programs' educational effectiveness (ACGME, 2006).

Within the Outcome Project there are six general competencies for residency education to ensure that residents are trained and develop inot 
competent, independent practitioners (Joyce, 2006, pg 10). These competencies were based on a national consensus on "what residents should know and be able to do" for board certification (ACGME, 2006, n.p.). Just as with the medical home and care coordination, the goal of the Outcome Project is the improved quality of patient care through resident education (ACGME, 2006). As outlined by the ACGME (2006), the six domains of the ACGME Competencies are as follows: Medical Knowledge, Patient Care, Professionalism, Interpersonal and Communication Skills, Practice-Based Learning and Improvement and SystemBased Practice (ACGME, 2006).

Each competency has specific requirements as well as guidelines for assessment. Some competencies, such as medical knowledge and patient care, have already been established within medical education in some form (Joyce, 2006).

Medical knowledge is much of the focus of the four years of medical school. By definition, medical knowledge requires the demonstration of knowledge about established and evolving biomedical, clinical, and cognate (e.g. epidemiological and social-behavioral) sciences and how to apply this knowledge to patient care (ACGME, 2006).

On the other hand, patient care focuses on interaction with individual patients and the community. Residents must be able to provide patient care that is compassionate, appropriate, and effective for the treatment of health problems and the promotion of health (ACGME, 2006). Both patient care and medical 
knowledge were identified within the ACGME training materials as not needing to be directly targeted within resident education (Joyce, 2006).

The main focus of the Outcomes Project appeared to be the remaining four competencies, professionalism, interpersonal skills and communication, practice-based learning and improvement and finally, system's based practice. Two of these four competencies, interpersonal skills and communication as well as professionalism, were previously considered as part of medical education. They have been since identified as needing specific attention and clarification (Joyce, 2006).

Examining the construct of professionalism, residents are to demonstrate respect, compassion, strong ethical principles and sensitivity to diversity when interacting with others (Joyce, 2006). The ACGME defines professionalism as the ability to investigate and evaluate their patient care practices, to appraise and assimilate scientific evidence, and then subsequently to improve their patient care practices (ACGME, 2006).

In contrast, interpersonal skills and communication encompasses communicating with patients including and beyond just history taking. Effective communication skills are at the heart of quality patient care. Residents are expected to communicate with others in a manner that is clear, effective, and empathetic (Joyce, 2006). Communication skills are especially important within care coordination teams. The outcome of these skills is the demonstration of effective information exchange and teaming with patients, their patient's families, and professional associates (ACGME, 2006; ACGME, 2007; Joyce, 2006). 
Therefore, residents need strong team communication and leadership skills in order to work effectively within a complex health care system.

The final two competencies are new educational expectations for residents (Joyce, 2006). They include practice based learning and improvement as well as systems-based practice (Joyce, 2006). These competencies not only emphasize continued and life-long improvement of patient care but also familiarity and responsiveness to the larger context, system and resources within the health care community (Joyce, 2006).

The first of these is practice-based learning and improvement. This competency teaches the discipline of life-long learning. The resident is to demonstrate practice-based learning and improvement through reflection and evaluation of their abilities to provide patient care, investigation and assimilation of new research and then systematic development of a quality improvement plan (Joyce, 2006; ACGME, 2006).

The other competency receiving more focus is system-based practice. This competency encompasses residents' ability to work in and incorporate the larger medical system/ community (ACGME, 2006; Joyce, 2006). The resident must demonstrate an awareness of and responsiveness to the larger context and system of health care and the ability to effectively call on system resources to provide optimal health care (Joyce, 2006; ACGME, 2006).

Systematic quality control is a required and important aspect of the Outcome project as it affects the educational system at the university level (ACGME, 2006; ACGME, 2007; Joyce, 2006). Medical schools must evaluate 
their educational program annually. They must document an annual meeting reviewing program goals and objectives as well as the effectiveness with which they are achieved (ACGME, 2006; ACGME, 2007; ACGME, 2010). When deficiencies are identified, an action plan is prepared (ACGME, 2006; ACGME, 2007). The program should use resident performance and outcome assessments to evaluate the educational effectiveness of the residency program (ACGME, 2006).

\section{Resident Training in the Community}

To improve child health at a community level, pediatricians need knowledge and skills not previously included within residency training (Lypson, Frohna, Gruppen, \& Woolliscroft, 2004). Education of residents of patient care beyond the hospital and clinical practice settings and within the community is in increasing demand (Lypson, et al., 2004 Shipley, et al., 2005). Recent policies from the AAP and requirements from ACGME Residency Review Committee also emphasize the importance of community training (Shipley, et al., 2005). Because teaching community pediatrics is relatively new, the challenge has been to define specific expectations and training goals to fulfill the needs within the community environment (Shipley, et al., 2005).

In looking at these expectations and training guidelines, the Pediatrics Residency Review Committee (RRC) of the ACGME requires community experiences as a core component of residency curricula (ACGME, 2007). The community experiences may include didactics but must involve residents in a

community-based experience (ACGME, 2007). In comparison, the training 
described by the Ambulatory Pediatric Association (APA) is more specific and comprehensive in its scope and description of community pediatric education (ACGME, 2007).

The American Academy of Pediatrics Future of Pediatric Education II (FOPE II) committee published a report that not only confirmed the need for education within ambulatory and community settings, but also described the role of the pediatrician generalist (ACGME, 2007). These roles include serving as community consultants, population-based community medicine practitioners, school-based pediatricians, and providers for home-based medical care for chronically ill children (ACGME, 2007). Whether these residents find themselves in a clinical, hospital, or community setting, they may all encounter a child's need for speech-language therapy or assistive technology.

\section{Current Research}

With the current educational expectations and literature available, the pediatric resident's knowledge regarding communication disorders and AAC should be sound. A review of literature has shown otherwise. Not only is the topic under-investigated, the findings were limited. For example, specific research regarding pediatric resident knowledge about communication disorders and AAC was conducted in the early 2000 . Sneed, a now retired pediatric rehabilitation specialist, and his colleagues carried out a series of empirical studies about the topic. In a 2000 study, Sneed, et al., conducted a survey of pediatric residents along with practicing pediatricians across 2 states. This study specifically investigated the preparation of physicians in practice and in training 
for the prescription of therapies, including speech therapy, and durable medical equipment, including communication devices.

The researchers found little literature describing the preparation of pediatricians to prescribe therapies and devices to CSHCN (Sneed, May, \& Stencel, 2000). The research results indicated about $70 \%$ of the respondents had no training in prescribing certain durable medical equipment (DME) and greater than $50 \%$ had no training in prescribing certain therapies (Sneed, et al., 2000). Twenty percent of the respondents reported a lack in training to treat some common childhood physical disabilities (Sneed, et al., 2000). Furthermore, the findings suggest a lack of physician and resident confidence in prescribing therapies and devices to CSHCN. Three-quarters of the respondents indicated that they did not believe that they were adequately prepared to take an active role in prescribing therapies and DME (Sneed, et al. 2000). Eighty percent of respondents reported no training in prescribing DME, including communication devices. Only $5 \%$ received greater than 1 hour of training in any category of equipment. Sneed, et al. (2000) stated that there was "a striking sense of inadequate training evidenced among residents as well as practicing physicians in each state for the various DME categories" (p.559). These results point to significant shortfalls of current educational system regarding training for the prescription of DME, and AAC in particular.

With the current expectations of the AAP for interdisciplinary team management, care coordination and team leadership are important aspects of educational training. Within Sneed et al.'s research (2000), $51 \%$ of residents 
reported no training on leading a team. Only $31 \%$ reported training time greater than one hour. Forty-two percent had training regarding prescribing speech therapy, with $25 \%$ being trained for longer than one hour. Only $19 \%$ of respondents felt they had adequate training regarding communication disorders.

In a follow-up study, Sneed, et al., (2004) considered the contribution of pediatricians towards the prescription and/or supervision of therapies and durable medical equipment (DME) within the child's medical and educational settings. The researchers conducted a quantitative survey presented to both pediatricians and pediatric residents. Information was gathered regarding how pediatricians and pediatric residents direct and coordinate therapy and DME for CSHCN. Their efforts were then compared with AAP recommendations at that time. An analysis of this study revealed that the majority of surveyed pediatricians do not regularly comply with AAP policy recommendations on prescribing therapy and DME in medical and educational settings. Interestingly, physicians who were trained before 1980 followed the AAP guidelines more closely than later graduates and current residents (Sneed, et al., 2000).

The results also indicated that there was decreasing involvement of private, outpatient pediatricians in coordinating and supervising CSHCN's care. This was despite increases in policies, such as the medical home and care coordination, requiring increased involvement in this setting. Furthermore, findings indicated that most treatment decisions were made by non-physician health care professionals versus the primary care pediatrician. 
An analysis of the results indicated that a little more than half of the pediatricians $(52.2 \%)$ surveyed stated that they would recommend professional services or therapy. When asked about speech therapy, $85.5 \%$ would give a communication disorder diagnosis. In providing specific treatment guidance, $42 \%$ would specify the frequency of treatment, $34 \%$ would prescribe the duration of treatment, $36 \%$ would specify goals, and $29.3 \%$ would give precautions for speech therapy intervention. When asked "if sent a prescription for therapy without your previous initiation would you sign the prescription if it was initiated by a speech-language pathologist "(p. 620), 70.8\% responded that they would (Sneed, et al., 2004). When services are provided, only $58 \%$ of pediatricians reported receiving a detailed progress report once or twice a year and one-fifth received no reports.

Other than team involvement, pediatricians and residents were questioned regarding their involvement in a child's school needs. Regarding participation in school IEPs, one-third said that they participated in educational services less than half the time. Fourteen percent stated that they never participated. Twentyfive percent of pediatricians or residents specify possible educational goals in their recommendations, and $76.7 \%$ prefer to let the therapists or educators set the goals. When asked about their review of individualized educational plan (IEP) materials from the educational team, $67.1 \%$ stated that they did review them. Only $52 \%$ reported subsequent follow-up progress information from the educational team. 
When comparing the results of the survey with AAP policies, the researchers found that only a minority of respondents fulfilled these expectations. The results of the data indicate that physicians presented diagnoses and not much else. Sneed et al., (2004) found the problems originating from physicians' lack of experience, lack of education, lack liability, and lack of communication in the team.

The researchers recommended that the AAP and other professional organizations (AMA and AAP Medicine and rehabilitation as well as federal guidelines and third-party payers) emphasize the role physicians have to initiate, identify, and order services within their ongoing patient treatment. The investigators argue that if a physician's authorization is required for reimbursement, then the physician's professional, legal and practice guidelines come into play (Sneed, et al., 2004). Recommendations for physicians included conscientiousness about fulfilling their responsibilities in serving as the medical home, and providing care coordination by supervising and monitoring medical services for their patients in both community and educational settings. Sneed and his colleagues suggested that failure to do so may bring significant consequences for the patient as well as subject the physician to legal liability (2004).

The report also pointed out the paradox regarding the increases in policies and recommendations, and diminished performance and involvement by pediatricians since 1980. In reviewing previous studies, Sneed et al. (2004) found little knowledge base of residents regarding CSHCN. The researchers 
suggested that information regarding therapist and DME should be included on board examinations to improve the knowledge base and required experience that pediatricians have regarding these topics.

\section{Summary}

Pediatricians play an important role in the lives of children with disabilities. Within their practice, pediatricians are to act as a medical home for children and take on the ethical responsibility to coordinate the care of a child up to age twenty-one (American Academy of Pediatrics, 2005; American Academy of Pediatrics, 1999; Brewer, McPherson, Magrab, \& Hutchins , 1989). Pediatricians must have knowledge of many aspects of a child's development or lack thereof, including a potential need for speech-language therapy and augmentative and alternative communication (AAC). Within the framework of the medical home, the pediatrician then takes on the role of care coordinator. According to new regulations by CMS and the Joint Commission, within a hospital or skilled nursing facility, pediatricians are to identify communication barriers. In both positions, pediatricians are to identify possible communication disorders followed by an appropriate referral for an evaluation by a certified speech-language pathologist (Desch, Gaebler-Spira, \& Disabilities, 2008).

In the 2008 clinical guide for Pediatrics, Desch, Gaebler-Spira, \& Disabilities, offered the premise that pediatricians should ensure access to appropriate augmentative and alternate communication services, including assessment, training, monitoring and funding. However, it is unknown to what degree pediatricians understand their responsibility. Additionally, it is unclear as 
to what level pediatricians know or understand augmentative and alternate communication systems and services.

In a previous assessment of resident knowledge of communication disorders and AAC in 2000 found that the majority of pediatric residents did not have knowledge about speech-language therapy, and they did not feel comfortable in prescribing communication devices (Sneed, May, \& Stencel, 2000). In 2004, Sneed, May, \& Stencel also found that pediatricians and pediatric residents were unsure of their role as care coordinators when prescribing therapies and durable medical equipment (DME). Only a minority of the survey respondents fulfilled the expectations of American Association of Pediatrics (AAP) policies (Sneed et al., 2004). Furthermore, their study indicated that physicians prescribed diagnoses and not much else. Reasons for these professional shortcomings included a lack of experience, a lack of education, a lack liability, and a lack of communication within the care coordination team (Sneed, et al., 2004).

To ensure the growing number of children with special health care needs ( $\mathrm{CSHCN}$ ) is being provided quality health care, a better understanding of the preparation of regarding prescribing the specialty therapies and durable medical equipment is needed. In order to better provide for individuals with communication disorders the level of training pediatricians in identifying communication disorders and the need for AAC appears to be paramount. 


\section{CHAPTER III}

\section{METHODOLOGY}

The purpose of this chapter is to describe the methods that were used in this study. The major areas that addressed include (a) survey development, (b) sample, (c) instrumentation, (d) questionnaire validations, (e) procedures, and (f) data analysis and reliability procedures.

\section{Survey Development}

The survey protocol (see Appendix C) was developed to conform to highest design principles in a manner consistent with Dillman, Smyth, Christian and Melani (2009). Additionally, the American Academy of Pediatricians policy regarding resident education, communication disorders and AAC was reviewed (e.g. AAP Committee on Children with Disabilities, 1998; Desch, et al., 2008; McPherson et al., 2004; Ziring et al., 1999) as well as surveys addressing graduate medical education(e.g. Sneed, et al., 2000; Sneed, et al., 2001; Sneed, et al., 2004; Sneed, et al., 2002). Through this review process, decisions were made regarding the form and content of the survey questions.

Question content was derived from the American Council of Graduate Medical Education (ACGME) educational competencies for pediatric graduate medical education (ACGME, 2006). Each competency listed in the ACGME Program Requirements for Graduate Medical Education in Pediatrics (2007) was 
reviewed for its relevance towards communication disorders and augmentative and alternative communication. The relevant items were formulated into questions specifically pertaining to communication disorders and augmentative and alternative communication.

Questions were developed to address the six ACGME competencies as well as research study constructs. Information regarding the alignment of each ACGME competency and each content question is provided in Appendix B. Information regarding the alignment of the research constructs and each content question can be found in Table 2 .

\section{Expert Panel Review}

The survey questions then underwent review by the members of the expert panel. Seven pediatricians served on the expert panel and assisted in the survey development. These pediatricians were selected for their expertise in developmental disabilities as well as their participation in resident education through the University of Louisville's Department of Pediatrics. After the initial content was identified from research on ACGME competencies, a draft of the survey was emailed and hand delivered to members of the expert panel. Written and verbal feedback was gathered from these individuals, and revisions and/ or corrections were made. The survey went through the revision process a total of four times before the expert panel presented its final approval.

\section{Instrumentation}

The survey, Survey of Pediatric Residents: Communication Disorders and Augmentative and Alternative Communication (AAC) (SPR:CDAAC), consisted of 
forty-five questions divided into (1) thirty-one questions pertaining to pediatric resident ACGME competency and (2) fourteen demographic questions. The thirty-one questions regarding pediatric resident ACGME competency covered the six ACGME competencies as well as research study constructs. Information regarding the alignment of each ACGME competency and each content question can be found in Appendix B. Information regarding the alignment of the research constructs and each content question is presented in Table 1 below.

Table 1

Survey questions aligned with research questions.

Research Questions

1. Is there a significant difference in

perceived pediatric resident educational

training experiences for communication

disorders and AAC across pediatric

levels?
Survey Questions

\section{$1,2,3,4,5$}

2. Is there a significant difference in perceived pediatric resident knowledge

of communication disorders and AAC

across pediatric levels?

3. Is there a significant difference in

perceived pediatric resident competency $12,13,14,15,16,17,18,19,20,21$, for professional practice regarding the $22,23,24,25,26,27,28,29,30,31$ care of children with communication 
disorders and AAC across pediatric levels?

4. What effects do demographic variables have on residents' perceived $32,33,34,35,36,37,38,39,40,41,42,43,44$ competency? , 45

The constructs used for the questions regarding competency were education, knowledge, and professional practice. As can be seen from the above, questions one to thirty-one addressed these constructs in-depth.

Education construct. The first construct was education. In general, education is training and instruction in a particular subject, or the imparting and acquiring of knowledge through teaching and learning (Encarta® World English Dictionary [North American Edition], 2009). Specifically within this study, education referred to graduate medical education. The period of education in a medical specialty following undergraduate medical education which prepares the physician for independent practice of that specialty (also referred to as residency education) (ACGME, 2009). This construct corresponded with the following ACGME competencies: Medical Knowledge, Patient Care, and Medical Education.

Knowledge construct. The next construct investigated was knowledge. Knowledge, as defined by the Encarta® World English Dictionary [North American Edition] (2009), as "a general awareness or possession of information, facts, ideas, truths, or principles" (p. 7b). This construct investigated resident 
knowledge of communication disorders and AAC in compliance with the ACGME competencies of Medical Knowledge, Patient Care and Medical Education.

Professional practice construct. The final construct addressed was professional practice. The American Board of Pediatrics (ABP) developed guidelines for the teaching and evaluation of professionalism, or professional practice, as part of the core curriculum for residency training in pediatrics (Fallat, \& Glover, 2007, pg. e1124). These guidelines overlap and accentuate five of the six ACGME competencies including the following: Patient Care, Professionalism, Interpersonal and Communication Skills, Practice-Based Learning and Improvement and System-Based Practice. Professional practice is distinctive from medical knowledge. The following eight components of professional practice are endorsed by the ABP: honesty and integrity, reliability and responsibility, respect for others, compassion/ empathy, self-improvement, selfawareness/ knowledge of limits, communication and collaboration and altruism and advocacy (Fallat, et al., 2007).

The final part of the instrument included fourteen demographic questions investigating the affect these differences may have on pediatric resident competency. The demographic information requested in the survey included the following: gender, specialization, pediatric level (graduate year), rotation completion, form and amount of education regarding communication disorders and $A A C$. 


\section{Content Validity}

To gather accurate data from the sample, the survey instrument was examined regarding its reliability and validity. Reliability was examined through assessing the instrument's internal consistency. Cronbach's alpha internal consistency reliability coefficients were calculated for each set of items in the questionnaire that were assumed to be measuring a scale. For example, all of the items under the Information Regarding Education section of the survey were examined to determine if they have sufficient reliability to be averaged for a single scale score. The minimum criterion used was .70 coefficient. Several measures were used to improve control within the study, such as having participants specify their years of resident training/ education. Additionally, the participants were participating in and receiving the same educational training with one university program, the University of Louisville's School of Medicine Pediatric residency program. All participants received the same survey. The survey was presented to all participants through the use of Survey Monkey ${ }^{\mathrm{TM}}$ (Survey Monkey, 2009). Finally, all survey information was coded to ensure participant privacy and ethical conduct in accordance to current HIPPA and IRB guidelines.

\section{Participants}

The participants for this study included all current pediatric medical residents within graduate medical training at the University of Louisville. Participant information was gathered at the same time for all pediatric levels. The population included all three years of residents. The total number of pediatric resident in the 2010-2011 academic year was 102 . That number 
includes 29 first-year residents, 24 second-year residents, and 26 third-year residents. Also included were five fourth-year residents completing their combined pediatrics and general medicine program. The last group was included within the third year of residency secondary to their general educational status. This brings the number of third-year residents to 31 . Human subject's consideration and clearance was obtained and documentation is provided within the Appendix (see Appendix document F).

\section{Procedures}

The implementation of this research was based on techniques of conducting an online survey as described by Dillman, et al. in Internet, Mail, and Mixed-Mode Surveys: The Tailored Design Method (2009). The following steps outline the specific procedures that were used.

A "roster" of the survey participants was developed. The roster included participant names and email addresses. An automated process through Survey Monkey ${ }^{\mathrm{TM}}$ was used to send a personalized email to each person on the roster (See Appendix I). The email explained the survey topic, the purpose of the survey (See Appendix I), definition of communication disorders and augmentative and alternative communication (AAC) and requested their participation. The email provided them a link to the survey webpage. Embedded in the link is a unique "key" that allowed the respondent to complete the survey only once.

Copies of the survey can be found in the Appendiices D and E. The survey collected quantitative data. Survey responses were confidential. Survey responses were kept on a password-protected secure on-line database provided 
by Survey Monkey ${ }^{\mathrm{TM}}$. Participation in the survey was voluntary. To be eligible to participate, the student were actively enrolled within the pediatric residency program and the University of Louisville.

When respondents clicked on the link to the survey page they were given a means to indicate consent for participation and authorization of their responses to be used within the study. They were also provided a means to decline participation in the survey. Survey Monkey ${ }^{\mathrm{TM}}$ built the survey page dynamically from a list of questions and response options that were stored in a secure database. All formatting was done automatically by the server. Once the survey window closed, the same server process automatically computed and formatted the results.

After respondents successfully completed a survey, their completion was automatically recorded on the roster, and their answers to the survey questions were recorded in a separate and un-connected table, thus, maintaining anonymity of the respondents. The table allowed the investigator to keep track of percentage of survey completion. Follow-up emails (See Appendix I) were sent two weeks after the first request. A thank-you message was sent to the individuals that fully completed the survey. A final follow-up email was sent two weeks to non-completers.

Upon completion of the survey, descriptive qualitative data was gathered from the survey population through the use of a focus group. The purpose of the focus group was to obtain pediatric resident's perceptions regarding 
communication disorders and $A A C$ as part of their training. The discussion was designed to obtain perceptions within a permissive, non-threatening environment.

To recruit individuals within the focus group, the "roster" of survey participants was used. An automated process through Survey Monkey ${ }^{\mathrm{TM}}$ was used to send a personalized email to each person on the roster. The email described the purpose of the focus group, defined communication disorders and augmentative and alternative communication (AAC) and requested their participation.

The exploratory focus group consisted of the moderator/ investigator, note-taker, and participants. A cassette tape recorder was used to record data for later transcription. The focus group discussed several general questions. One was regarding the survey and others were regarding the current and future roles the individuals will play in dealing with communication disorders and AAC. Logical follow-up was used after participants responded to the general questions. The incentive used for participation in the online survey was a chance of winning a gift bag with 200 dollars of gift cards from local merchants. The incentive for participation in the focus group was a free lunch provided for all participants.

\section{Data Analysis}

\section{Research Questions 1 - 3}

Data pertaining to three of the four research questions were analyzed using a one-factor multivariate analysis of variance. Each question pertained to a specific construct within the dependent variable. The three constructs and their research questions were: 
a) Medical Education - Was there a significant difference in perceived pediatric resident educational training experiences for communication disorders and $A A C$ across pediatric levels?

b) Medical Knowledge - Was there a significant difference in perceived pediatric resident knowledge of communication disorders and AAC across pediatric levels?

c) Professional Practice - Was there a significant difference in perceived pediatric resident competency for professional practice regarding the care of children with communication disorders and AAC across pediatric levels?

The independent variable was education level of resident (three levels, from 1 to 3 years). The three dependent variables were the average scores on the three constructs obtained from the questionnaire:

$\mathrm{a}=$ perceived pediatric resident educational training experiences (Medical Education)

$b=$ perceived pediatric resident knowledge (Medical Knowledge).

$\mathrm{C}=$ perceived pediatric resident competency for patient care (Professional Practice)

The statistical procedure used was a one-factor multivariate analysis of variance (MANOVA). MANOVA was used to determine if significant differences exist among the three groups of residents in the average levels of self-reported competence in the three constructs regarding communication disorders and augmentative and assistive technology. The ability of the data to meet 
methodological assumption was assessed. The assumptions for a MANOVA included independence, multivariate normality, and equality of variance covariance matrices If the MANOVA was statistically significant, Tukey multiple comparisons were performed on the means from each dependent variable.

Statistical Package for the Social Sciences (SPSS) version 15 Windows (SPSS, 2003), was used for data entry as well as computational analysis. All statistical analyses used .05 as the level of significance.

\section{Research Question 4}

The fourth research question investigated the effects of demographic variables on residents' perceived competency. Independent $t$ tests and correlation coefficients were used to analyze the data. The dependent variables were residents' perceived competency (the average scores on the three constructs obtained from the questionnaire) and the independent variables were demographic variables within the survey.

The purpose of the $t$ tests determined if significant differences existed between the participants in the two levels of each independent variable on the three competency scores derived from the questionnaire. The objective of the correlation coefficients was to determine the strength of linear relationship between the competency scores and a demographic variable dealing with time spent learning about communication disorders and AAC.

a. Independent variable, gender: Males and females were compared on the average levels of self-reported competence in the three constructs 
regarding communication disorders and augmentative and assistive technology.

b. Independent variable, pediatric specialization: Various specializations were coded as either Yes or No and the two groups were compared on the average levels of self-reported competence in the three constructs regarding communication disorders and augmentative and assistive technology.

c. Independent variable, rotation completion: participants were coded as either Yes or No and the two groups will be compared on the average levels of self-reported competence in the three constructs regarding communication disorders and augmentative and assistive technology. d. Independent variable, educational methods: coded into two groups and the average levels of self-reported competence in the three constructs regarding communication disorders and augmentative and assistive technology. Educational methods included one or more of the following: ambulatory pediatric rotation, subspecialty rotation, morning report, core conference, didactic or board conference.

e. Independent variable, educational time: Educational time was the amount of time a pediatric resident received instruction through the above educational methods. The variable educational time was summed for each participant and correlated with the average levels of self-reported competence in the three constructs regarding communication disorders and augmentative and assistive technology. 
Coded data were used for the several of the above $t$ tests. For example, for gender, male was coded as ' 1 ', and female was coded as ' 0 '. Pediatric specialization, rotation completion and education methods also used a similar coding system with 'yes' coded as ' 1 ' and 'no' coded as ' 0 '. Educational time was the only measure that was not coded. The average of the sum total was used as one of the variables in a set of correlation coefficients.

Statistical Package for the Social Sciences (SPSS) version 15 Windows (SPSS, 2003), provided the computational analysis. All statistical analyses used .05 as the level of significance. However, to obviate the inflation of Type I error rate due to repeated statistical tests, the Bonferroni correction was used. This correction method was implemented in the following manner. A set of tests associated with one of the independent variables was defined as a set. Within this set, the overall error rate was kept at .05 by dividing the number of tests into .05 and using the resulting value as the alpha level to be used for each comparison. For example, the first demographic variable was gender. This yielded three independent $t$ tests (one for each subtest derived from the questionnaire). Since $.05 / 3=.017$, the value .017 would be used as the criterion of statistical significance for each $t$ test.

The ability of the data to meet methodological assumption was assessed. The assumptions for an independent $t$ test included independence, normality, and equality of variances. For correlation coefficients, a linear relationship between two variables being correlated was determined. 


\section{Research Question 5}

The fifth question investigated the perspectives of pediatric residents regarding communication disorders and $A A C$ as part of their training. This question provided qualitative data gathered through responses to several openended questions. The following questions were used:

1. What are your thoughts about the survey regarding communication disorders and AAC?

2. How do you perceive your current role regarding communication disorders and AAC?, and

3. How do you perceive your future role regarding communication disorders and $A A C ?$

The qualitative information gathered through the open-ended questions was read, transcribed and coded. Comments were organized into similar categories and the categories were labeled as specific conversational topics. The investigator attempted to identify patterns, or associations in the topics. The investigator used reflexivity to keep possible personal bias from entering the analysis of the qualitative data. The personal values, ideas and pre-judgments of the examiner were recorded and addressed as needed. 


\section{CHAPTER IV}

\section{RESULTS}

This survey investigated the current level of perceived competency of residents within and across pediatric levels. The data was analyzed for significant changes in perceived competency of pediatric residents regarding their medical knowledge, professional practice and education regarding communication disorders and AAC over the course the three year pediatric residential program at the University of Louisville.

The purpose of this chapter is to present the results of the study obtained through the quantitative analyses of the survey data and the qualitative analysis of the focus group discussion. This chapter is divided into the following sections: (a) data checking and coding, (b) demographic variables, (b) instrument reliability and validity, (c) results from the research questions, and (d) a summary.

\section{Data Collection}

Data for the study were obtained from two sources; a survey of current Pediatric Residents on staff with the University of Louisville, and an interview conducted as a focus group. An invitation to the survey, Survey of Pediatric Residents: Communication Disorders and Augmentative and Alternative Communication (PR:CDAAC), was sent to 102 Pediatric Residents. The Survey Monkey ${ }^{\mathrm{TM}}$ distribution system was used to send out the invitation for the survey 
to all potential participants on November, 29th, 2010. See Appendix, I for a copy of the email. Several participants contacted the investigator regarding an error in the response format of the survey the day of its initial distribution. The help line for Survey Monkey ${ }^{\top \mathrm{M}}$ was contacted by the investigator and a solution was found the same day. The responses by the previous individuals had to be identified and deleted. A subsequent email with both an apology and request to reenter their responses was sent (see Appendix I). The potential participants who had not completed the survey were not affected by this error and did not receive the second email. The initial response to the survey invitation was five participants.

Due to the holiday season and upcoming vacations, the timeline for followup emails was expedited. December $1^{\text {st }}, 97$ emails were sent to all nonresponders, again using Survey Monkey's ${ }^{\mathrm{TM}}$ distribution system. Table 2 shows the date of each email along with the number of responders per distribution. Table 2

Study Response Rate/ Per Request

\begin{tabular}{clcc} 
Date & Population Mailing & $\#$ & $\%$ Response \\
\hline November 29 & 102 & 5 & $5 \%$ \\
December 1 & 97 & 19 & $24 \%$ \\
December 10 & 78 & 14 & $37 \%$ \\
December 15 & 64 & 8 & $45 \%$ \\
December 21 & 56 & 0 & $45 \%$ \\
TOTAL & $46(43$ completers) & & $42 \%$ \\
\hline
\end{tabular}

The final request for participation was sent on December 21, 2010. See 
Appendix I for a copy of this email. Within this email, the end date for participation was identified as December 28th, 2010. A timeline was also given for when the selection for the prize drawing would be made along with information regarding prize notification. Forty-three participants responded with two opting out and one non-completer. A total of $42 \%$ of the population participated. On December 29th, all the names of the survey participants were placed randomly within a container and one winner was drawn. An email was sent to this individual to notify them of their prize winnings (see Appendix I). The individual picked up their winnings on January, $12^{\text {th }} 2011$.

An emailed invitation was sent for participation in the focus group on December $6^{\text {th }}, 2010$. Notification for the December 13 th focus group was distributed using the Survey Monkey ${ }^{\mathrm{TM}}$ list serv. See Appendix I for a copy of the email. The sixth floor conference room within Kosair's Children's Hospital was used due to its familiarity with the residents, and its use on Mondays for educational conference sessions. The investigator provided a free lunch for focus group participants. The conference room was set up with cassette tapes placed throughout the room and consent for participation and authorization forms placed at the door. One-hundred and two individuals were invited to participate within the focus group. Four individuals participated. All pediatric levels were represented; one participant in PL1, two participants in PL 2 and one participant in PL3. There was equal gender representation with two females and two males. Unknown to the investigator, another conference was scheduled for the same day at another location. 
Interview information was recorded through cassette tape and transcribed for analysis. The focus group discuss lasted for 45 minutes. Cassette tapes and consent for participation and authorization forms for comments to be used within the study were placed in a secure, locked drawer in the investigator's office.

\section{Missing Values}

One response out of 31 was missing for items Q12R, Q24R, and Q30R. The missing values for all of these factors were replaced with the mean value. Forty-two participants completed the survey with one noncompleter. Two individuals opted out of the survey, and four individuals participated in the focus group. All focus group participants had indicated that they had completed the survey.

\section{Data Coding}

Quantitative information. All information was entered into a SPSS database, being attentive to level of measurement in the process. Data for all variables and subjects were converted to numerical values then entered into the SPSS database management program, see Appendix C. Frequency tables were used to confirm all data was properly coded and categorized.

Qualitative information. The qualitative information gathered through the open-ended questions was read, transcribed and coded. Comments were organized into similar categories and the categories were labeled as specific conversational topics. The investigator attempted to identify patterns, or associations in the topics. The investigator used reflexivity to keep possible personal bias from entering the analysis of the qualitative data. The personal 
values, ideas and pre-judgments of the examiner were recorded and addressed as needed.

\section{Descriptive Statistics}

Descriptive statistics for this study include measures of central tendency: the Mean for the group and the Percentage. Descriptive statistics are used within the independent variables for research questions one through thirty-one investigating changes in resident perceptions of competency over education and research questions thirty-two through forty-five investigating the affect these differences may have on pediatric resident competency. The demographic information requested in the survey included the following: gender and pediatric level (graduate year). Other descriptive statistics within this section included the following: specialization, rotation completion, and the form and amount of education regarding communication disorders and AAC. Coding of variables can be found in Appendix $C$.

\section{Key Demographic Variables}

Demographic Controls had two sections: (a) Gender (GEN) and (b) Pediatric Level (PL). Table 3 presents the gender distribution for the pediatric residents who participated in the PR:CDAAC survey. As can be seen, more females completed the survey than males. Females represented $60.5 \%$ of the respondents, and males represented 39.5 percent. 
Table 3

Descriptive Statistics for Gender and PLs $(N=43)$

Measure $\quad$ Frequencies Percentages

GEN

$\begin{array}{lll}\text { Female } & 26 & 60.5 \% \\ \text { Male } & 17 & 39.5 \%\end{array}$

PL $(1-3)$

$\begin{array}{lll}\text { PL } 1 & 15 & 34.9 \%\end{array}$

$\begin{array}{lll}\text { PL } 2 & 11 & 25.6 \%\end{array}$

PL $3 \quad 17 \quad 39.5 \%$

Note. $\mathrm{PL} 1$ = pediatric level one; $\mathrm{PL} 2$ = pediatric level $2 ; \mathrm{PL} 3=$ pediatric level 3.

Table 3 also shows the pediatric levels represented. The response rate

for PL1 and PL3 were close in frequency with 15 (34.9\%), and 17 (39.5\%)

participants. PL2 had the smallest representation with 11 (25.6\%) participants.

Confounding factor. The original number of pediatric residents presented to the researcher was 86 with a roughly equal number between pediatric levels, $\mathrm{PL} 1=29, \mathrm{PL} 2=24$ and $\mathrm{PL} 3=26$ with an addition of five fourth year residents ( $P L 3$ total $=31$ ). When the distribution list was presented to the investigator, it was noted that there was a significant difference in PL 3. The number of PL 3 significantly increased to 49 . The cause for the increase was a number of pediatric residents continuing their education within various specializations beyond four years. For example, there were several sixth year residents listed specializing emergency department. 


\section{Additional Descriptive Data}

Additional descriptive data was collected regarding three other aspects of pediatric residency: Specialization (SPEC), Rotation Completion (RC) and Educational Experiences for both Communication Disorders (EECD) and AAC (EEAAC).

Specialization. Within Pediatrics various specializations are pursued to acquire board certification for practice in a particular area. This specialization may influence educational practice, and was therefore identified as a needed descriptive statistic. Information regarding specializations (SPEC) was analyzed for pediatric residents. Table 4 shows the number of pediatric residents pursuing a specialization. As can be seen in Table 4, 55.8\% of the pediatric residents completing the survey were pursuing a specialization.

Table 4

Descriptive Statistics for Specialization $(N=43)$

Measure Frequencies Percentages

SPEC

$\begin{array}{lll}\text { YES } & 24 & 55.8 \%\end{array}$

$\frac{\mathrm{NO}}{\text { Note. PL 1 = pediatric level one; PL 2 = pediatric level 2; PL 3 = pediatric level } 3 .}$

Further analysis regarding specific specializations was assessed. The following specializations were reported: allergy and Immunology (Al), Critical Care Medicine (CC), Emergency Medicine (EM), Endocrinology (EN), General Pediatrics (community practice) (GP), Hematology/ Oncology and Bone Marrow Transplant (HOBBMT), Infectious Diseases (ID), Medical Genetics (MG), 
Neonatal Medicine (NM), Other (O).

Table 5

Descriptive Statistics for Specific Specializations $(N=24)$

\begin{tabular}{lcr} 
Measure & Frequencies & Percentag \\
\hline SPEC & 16 & $37.2 \%$ \\
AI & 2 & $4.7 \%$ \\
CC & 4 & $9.3 \%$ \\
EM & 3 & $7.0 \%$ \\
EN & 2 & $4.7 \%$ \\
GP & 3 & $7.0 \%$ \\
HOBBMT & 2 & $4.7 \%$ \\
ID & 1 & $2.3 \%$ \\
MG & 1 & $2.3 \%$ \\
NM & 7 & $16.3 \%$ \\
O & 2 & $4.7 \%$ \\
\hline
\end{tabular}

Note. $\mathrm{Al}=$ allergy and Immunology, $\mathrm{CC}=$ Critical Care Medicine, $\mathrm{EM}=$ Emergency Medicine, EN = Endocrinology, GP = General Pediatrics (community practice), HOBBMT = Hematology/ Oncology and Bone Marrow Transplant, ID = Infectious Diseases, $M G=$ Medical Genetics, $N M=$ Neonatal Medicine, $\mathrm{O}=$ Other

As can be noted from the above, the number of residents indicating a 'yes' to pursuit of a specialization, 24 , does not equal the number of individuals identifying their specific specialization, a total of 27 . This difference can only be identified as responder error. Eleven individuals skipped this question indicating that they are not pursuing a specialization. Ten specializations were identified out of the list of 24 provided. The 14 specializations that are currently not being pursued include the following: adolescent medicine, ambulatory pediatrics, 
cardiology, developmental/ behavioral pediatrics, forensic medicine, gastroenterology, general inpatient medicine (hospitalists), international pediatrics, medical history, ethics and humanities, nephrology and hypertension, pulmonary medicine, radiology, rheumatology, and sleep medicine. The specialization most frequently identified was neonatal medicine (NM), $16.3 \%$, with critical care (CC) coming in second, $9.3 \%$. The two lowest specializations included Infectious Diseases (ID), 2.3\%, and Medical Genetics (MG), 2.3\%.

Rotation completion. Throughout their training, pediatric residents complete various rotations as part of their education. Specific rotations are completed as part of each pediatric level and may influence educational training. Many rotations are mandatory, but some are selected as part of a medical specialization. Completion of rotations was therefore identified as in of a need descriptive statistic. Information regarding the following rotations (ROT) was reported:

Table 6

Descriptive Statistics for Rotation completion $(N=43)$

\begin{tabular}{ccc}
\hline Measure & Frequencies & Percentages \\
\hline ROT & 30 & $69.8 \%$ \\
AM & 17 & $39.5 \%$ \\
APS & 9 & $20.9 \%$ \\
Al & 17 & $39.5 \%$ \\
CAR & 5 & $11.6 \%$ \\
CDV & & table continues on the next page
\end{tabular}


Table 6 continued

\begin{tabular}{lcc} 
Measure & Frequencies & Percentages \\
\hline CHP & 4 & $9.3 \%$ \\
CCM & 15 & $34.9 \%$ \\
END & 1 & $2.3 \%$ \\
EM & 12 & $27.9 \%$ \\
F & 35 & $81.4 \%$ \\
G & 2 & $4.7 \%$ \\
GEN & 19 & $44.2 \%$ \\
HO & 10 & $23.3 \%$ \\
IPS & 26 & $60.5 \%$ \\
ID & 38 & $88.4 \%$ \\
NEO & 19 & $44.2 \%$ \\
NEPH & 38 & $88.4 \%$ \\
NEUR & 13 & $30.2 \%$ \\
NEW & 13 & $30.2 \%$ \\
P & 16 & $72.1 \%$ \\
PICU & 31 & $0.0 \%$ \\
PC & 0 & $37.2 \%$ \\
PUL & 16 & $91.9 \%$ \\
R & $16.6 \%$ \\
& & table continues on the next page \\
& 13 &
\end{tabular}


Table 6 continued

Measure

RHE

SSC

PPO

RES
Frequencies

4

7

12

4

5
Percentages

$9.3 \%$

$16.3 \%$

$27.9 \%$

$9.3 \%$

\section{WCEC}

$11.6 \%$

Note. $\mathrm{AM}=$ Adolescent medicine, $\mathrm{APS}=$ Ambulatory pediatrics services, $\mathrm{Al}=$ Allergy/ immunology, CAR = Cardiology, CDV = Child development, $\mathrm{CHP}=$ Community health programs, $\mathrm{CCM}=$ Critical care medicine, $\mathrm{D}=$ Dermatology, $\mathrm{END}=$ Endocrinology, $\mathrm{EM}=$ Emergency Medicine, $\mathrm{F}=$ Forensics, $\mathrm{G}=$ Gastroenterology, GEN = Genetics, $\mathrm{HO}=$ Hematology/ Oncology, IPS = InPatient service (Wards), ID = Infectious diseases, NEO = Neonatology, NEPH = Nephrology, NEUR = Neurology, NEW = Newborn, $\mathrm{P}=$ Pathology, $\mathrm{PICU}=$ Pediatrics ICU, PC = Primary care, $\mathrm{PUL}=$ Pulmonology, $\mathrm{R}=$ Radiology, $\mathrm{RHE}=$ Rheumatology, SSC = Special surgical clinics, $\mathrm{PPO}=$ Private practitioner's offices, RES = Research Activity, and WCEC = Weisskopf Child Evaluation Center (WCEC)

As can be seen from Table 6 , rotation participation ranges from $0 \%$ to $88.4 \%$. The rotations receiving the highest participation included the following: adolescent medicine (AM), 69.8\%, emergency medicine (EM), 81.4\%,in-patient services (Wards)(IPS), 88.4\%, neonatal (NEO), 88.4\%, hematology/ oncology (HO), 60.5\%, and newborns (NEW), 72.1\%. The rotations receiving the lowest participation included the following: child development (CDV), $11.6 \%$, community health programs (CHP), 9.3\%, dermatology (D), 2.3\%, forensics (F), $4.7 \%$, pathology $(P), 0.0 \%$, radiology $(R), 9.3 \%$, rheumatology (RHE), 9.3\%, research activity (RES), 9.3\% and, Weisskopf Child Evaluation Center (WCEC), $11.6 \%$. 
Rotations per PL. Since each PL completes the various rotations in a specific order, each PL's rotation information will also be reviewed in a comparison chart, see Table 7.

Table 7

Descriptive Statistics across PLs for Rotation completion $(N=43)$

$$
\underline{P L 1}(N=15) \quad \underline{P L 2}(N=11) \quad \underline{P L} 3(N=17)
$$

Measure

ROT

$\begin{array}{lcccccc}\text { AM } & 8 & 53.3 \% & 8 & 72.7 \% & 14 & 82.4 \% \\ \text { APS } & 0 & 0.0 \% & 4 & 36.4 \% & 13 & 76.5 \% \\ \text { Al } & 1 & 6.7 \% & 2 & 18.2 \% & 6 & 35.3 \% \\ \text { CAR } & 3 & 20.0 \% & 5 & 45.5 \% & 9 & 52.9 \% \\ \text { CDV } & 0 & 0.0 \% & 0 & 0.0 \% & 5 & 29.4 \% \\ \text { CHP } & 0 & 0.0 \% & 0 & 0.0 \% & 4 & 23.5 \% \\ \text { CCM } & 0 & 0.0 \% & 1 & 9.1 \% & 14 & 82.4 \% \\ \text { D } & 0 & 0.0 \% & 1 & 9.1 \% & 0 & 0.0 \% \\ \text { END } & 2 & 13.3 \% & 1 & 9.1 \% & 9 & 52.9 \% \\ \text { EM } & 9 & 60.0 \% & 11 & 100.0 \% & 15 & 88.2 \% \\ \text { F } & 1 & 6.7 \% & 0 & 0.0 \% & 1 & 5.9 \% \\ \text { G } & 4 & 26.7 \% & 8 & 72.7 \% & 7 & 41.2 \% \\ \text { GEN } & 3 & 20.0 \% & 1 & 9.1 \% & 6 & 35.3 \% \\ \text { HO } & 7 & 46.7 \% & 8 & 72.7 \% & 11 & 64.7 \% \\ \text { ID } & 2 & 13.3 \% & 8 & 72.7 \% & 9 & 52.9 \% \\ & & & & \text { table continues on the next page }\end{array}$


Table 7 continued

$$
\underline{P L 1(N=15)} \quad \underline{P L 2}(N=11) \quad \underline{P L} 3(N=17)
$$

\begin{tabular}{lcccccc} 
Measure & $\#$ & $\%$ & $\#$ & $\%$ & $\#$ \\
\hline IPS & 12 & $80.0 \%$ & 11 & $100.0 \%$ & 15 & $88.2 \%$ \\
NEO & 11 & $73.3 \%$ & 10 & $90.9 \%$ & 17 & $100.0 \%$ \\
NEPH & 1 & $6.7 \%$ & 3 & $27.3 \%$ & 9 & $52.9 \%$ \\
NEUR & 1 & $6.7 \%$ & 2 & $18.2 \%$ & 10 & $58.8 \%$ \\
NEW & 5 & $33.3 \%$ & 11 & $100.0 \%$ & 15 & $88.2 \%$ \\
P & 0 & $0.0 \%$ & 0 & $0.0 \%$ & 0 & $0.0 \%$ \\
PICU & 0 & $0.0 \%$ & 1 & $9.1 \%$ & 15 & $88.2 \%$ \\
PC & 3 & $20.0 \%$ & 4 & $36.4 \%$ & 11 & $64.7 \%$ \\
PUL & 3 & $20.0 \%$ & 5 & $45.5 \%$ & 6 & $35.3 \%$ \\
R & 0 & $0.0 \%$ & 1 & $9.1 \%$ & 3 & $17.6 \%$ \\
RHE & 1 & $6.7 \%$ & 1 & $9.1 \%$ & 2 & $11.8 \%$ \\
SSC & 1 & $6.7 \%$ & 2 & $18.2 \%$ & 4 & $23.5 \%$ \\
PPO & 1 & $6.7 \%$ & 2 & $18.2 \%$ & 9 & $52.9 \%$ \\
RES & 0 & $0.0 \%$ & 1 & $9.1 \%$ & 3 & $17.6 \%$ \\
WCEC & 0 & $0.0 \%$ & 0 & $0.0 \%$ & 5 & $29.4 \%$
\end{tabular}

Note. $\mathrm{AM}=$ Adolescent medicine, $\mathrm{APS}=$ Ambulatory pediatrics services, $\mathrm{Al}=$ Allergy/ immunology, CAR = Cardiology, CDV = Child development, $\mathrm{CHP}=$ Community health programs, $C C M=$ Critical care medicine, $D=$ Dermatology, $\mathrm{END}=$ Endocrinology, $\mathrm{EM}=$ Emergency Medicine, $\mathrm{F}=$ Forensics, $\mathrm{G}=$ Gastroenterology, GEN = Genetics, $\mathrm{HO}=$ Hematology/ Oncology, IPS = InPatient service (Wards), ID = Infectious diseases, NEO = Neonatology, NEPH = Nephrology, NEUR = Neurology, NEW = Newborn, $\mathrm{P}=$ Pathology, $\mathrm{PICU}=$ Pediatrics ICU, PC = Primary care, $\mathrm{PUL}=$ Pulmonology, $\mathrm{R}=$ Radiology, $\mathrm{RHE}=$ Rheumatology, $\mathrm{SSC}=$ Special surgical clinics, $\mathrm{PPO}=$ Private practitioner's 
offices, RES $=$ Research Activity, and WCEC $=$ Weisskopf Child Evaluation Center (WCEC).

As can be seen from the table above, each subsequent PL has an increase in the completion of various rotations. Within PL 1, 10 rotations were not completed by any of the residents. Within PL 2, 5 rotations were not completed by any of the residents, and in PL 3,2 rotations were not completed by any of the residents. The percentage of participation increases over each PL level as well.

Education. Pediatric residents partake in a variety of educational experiences. Educational methods may influence educational outcomes. Thus educational training methodologies were identified as being in need descriptive statistic analysis. The first aspect of educational training was addressed by the question, 'Have you completed rotations where communication disorders were discussed?' Table 8 presents the response to this question for both communication disorders and AAC.

Table 8

Descriptive Statistics for Education: Educational Discussion $(N=43)$

Measure Frequencies Percentages

Communication Disorders

Yes

No

AAC

Yes

No
$70.6 \%$

$29.4 \%$

5

$47.1 \%$

$52.9 \%$ 
Note. Forty-three individuals responded to this question.

As can be seen in Table 8, twelve individuals out of 43 indicated that communication disorders had been discussed within one of their rotations. Eight individuals out of 43 indicated that $A A C$ had been discussed within one of their rotations.

Confounding factor. One factor that needs to be addressed is the number of individuals reporting 'no' to the discussion of communication disorders and $A A C$ within their rotations. The item was stated in the following manner for both communication disorders and AAC. "Have you completed rotations where AAC was discussed? * If 'No' skip to \# 10."; "Have you completed rotations where AAC was discussed? * If 'No' skip to \# 13." Skipping this question indicated a 'no' response. Some individuals still responded to the question and marked 'no' instead of 'yes.' The question could have been better formatted for less confusion and potential error.

Information regarding the following educational methods for communication disorders (CDED) and AAC (AACED) was reported: Ambulatory pediatric rotation (APR), Subspecialty rotation (SR), Morning report (MR), Core conference (CORE), Didactic (DID), and Board Conference (BC). 
Table 9

Descriptive Statistics for Education: Educational Method ( $N=43)$

Measure

CDED

APR

SR

MR

CORE

DID

$\mathrm{BC}$

AACE

APR

SR

MR

CORE

DID
Frequencies 7

5

4

3

4

5

3

3

1

3

3

3

4
Percentages $58.3 \%$

$41.7 \%$

$33.3 \%$

$25.0 \%$

$33.3 \%$

$41.7 \%$

$25.0 \%$

$30 \%$

$10 \%$

$30 \%$

$30 \%$

$30 \%$

$40 \%$

$\begin{array}{lll}\text { BC } & 2 & 20 \%\end{array}$

Note. $\mathrm{APR}=$ Ambulatory pediatric rotation, $\mathrm{SR}=$ Subspecialty rotation, $\mathrm{MR}=$ Morning report, CORE $=$ Core conference, $\mathrm{DID}=$ Didactic, and $\mathrm{BC}=$ Board Conference.

As can be seen from Table 9, of the 43 survey participants, regarding communication disorders, 12 responded and five skipped the question. For the same question regarding $A A C$, ten participants responded and 34 skipped the question.

All educational methods take time. A frequency distribution categorizing 
the range of educational time spent on the topics of communication disorders and $A A C$ is presented in Table 10.

Table 10

Descriptive Statistics for Education: Hours Communication Disorders $(N=17)$ and $A A C(N=11)$

\begin{tabular}{lllllr} 
& \multicolumn{2}{c}{ Hours CD } & & \multicolumn{2}{c}{ Hours AAC } \\
\cline { 2 - 3 } Measure & $N$ & & & $N$ & $\%$ \\
\hline$<1$ hour & 2 & $11.8 \%$ & 2 & $18.2 \%$ \\
1 hour & 5 & $29.4 \%$ & & 4 & $36.4 \%$ \\
2 hours & 5 & $29.4 \%$ & & 3 & $27.3 \%$ \\
3 hours & 2 & $11.8 \%$ & & $9.1 \%$ \\
4 hours & 2 & $11.8 \%$ & & 1 & $9.1 \%$ \\
Other hours & 1 & $5.9 \%$ & & 0 & $0.0 \%$
\end{tabular}

Note. $\mathrm{CD}=$ Communication Disorders

A mediating factor for both sets of data is the diminished number of responses. For communication disorders, 17 responded with 27 skipping the question. For $\mathrm{AAC}, 11$ responded with 33 skipping the question. As can be seen from the above, the majority of responders $(10 / 17,58.8 \%)$ indicated that they received one to two hours of instruction on communication disorders. The majority of responders for $\operatorname{AAC}(7 / 11,63.7 \%)$ indicated that they also received one to two hours of instruction time regarding AAC.

\section{Instrument Validity and Reliability}

To gather accurate data from the sample, the survey instrument was examined regarding its reliability and validity for the three constructs within the dependent variable, Medical Education, Medical Knowledge and Professional Practice. Reliability was examined through assessing the instrument's internal 
consistency. Cronbach's (1951) coefficient alpha was computed for internal reliability of the quantitative portion of the PR:CDAAC Survey. Cronbach's alpha internal consistency reliability coefficients were calculated for each set of items in the questionnaire that were assumed to be measuring a construct. For example, all of the items under the Education were examined to determine if they have sufficient reliability to be averaged for a single scale score. The minimum criterion used was a .70 coefficient, but a relaxed level of significance (.6) can be utilized for exploratory research (Stevens, 2002).

\section{Instrument Validity}

The survey content was validated through subject matter experts who were selected based on their experience in developmental pediatrics. Further description of the instrument's development and validity are described in Chapter 3, Methods.

\section{Instrument Reliability}

Once all the data from the study were collected, the construct scales in the instrument (Medical Education, Medical Knowledge and Professional Practice) were assessed for reliability through an analysis of inter-item consistency. The purpose of the reliability analyses was to determine if items in each construct measured the same concept (Nunnally \& Bernstein, 1994). For the three constructs used in the study, Cronbach's alpha internal consistency reliability coefficient was computed. Table 11 shows the alpha coefficients. Each of these exceeded the criterion of .70 that is the minimum acceptable value for research (Nunnally \& Bernstein, 1994). 
Table 11

Cronbach's Alpha Internal Consistency Reliability Coefficients: Study Constructs

Scale Number of Items

Cronbach's Alpha Coefficient

\begin{tabular}{lcc}
\hline Medical Education & 5 & .86 \\
Medical Knowledge & 6 & .71 \\
Professional Practice & 21 & .94 \\
\hline
\end{tabular}

Further detailed analysis of each constructs' analysis is provided in Appendix B. This Appendix provides a description of each construct and a detailed listing of the Cronbach's alpha scores.

Correlation study. A correlation study was conducted to investigate the constructs used within the study. These were noted as follows: Medical Education (MED), Medical Knowledge (MK), and Professional Practice (PP).

Table 12

Correlations Between Constructs $(N=43)$

\begin{tabular}{lccc} 
Variable & MED & MK & PP \\
\hline MED & -- & $.59^{\star \star}$ & $.67^{\star \star}$ \\
MK & & -- & $.68^{\star \star}$ \\
PP & & --
\end{tabular}

Note. MED = Medical Education, MK = Medical Knowledge, $\mathrm{PP}=$ Professional Practice.

Note. ${ }^{*} p<.05 .{ }^{* *} p<.01$ (two tailed).

As can be seen from the above, the constructs were significant for interrelation. Medical Education and Medical Knowledge were moderately correlated, $n(43)=.59, p<.01$. Medical Education and Professional Practice 
were moderately correlated with the following significant results: $r(43)=.67, p<$ .01. Finally, Medical Knowledge and Professional Practice were also moderately correlated, $r(43)=.68, p<.01$.

\section{Results of Research Questions}

Four empirical questions and one qualitative question guided this research. The specific variables and types of statistical calculations used for each research question are described in Chapter III. The findings are reported by research question; the specific type of analysis for each research question was specified in Chapter III and is addressed under each.

\section{Research Questions 1 - 3: MANOVA}

A multivariate analysis of variance (MANOVA) was run on the data to answer the first three of the five research questions. Survey questions one to thirty-one addressed research question one through three. Each survey question pertained to a specific construct within the dependent variable. The three constructs and their research questions were as follows: (a) Medical Education; Was there a significant difference in perceived pediatric resident educational training experiences for communication disorders and $A A C$ across pediatric levels? (b) Medical Knowledge; Was there a significant difference in perceived pediatric resident knowledge of communication disorders and AAC across pediatric levels? (c) Professional Practice; Was there a significant difference in perceived pediatric resident competency for professional practice regarding the care of children with communication disorders and AAC across pediatric levels? 
The MANOVA test was utilized to compare group mean scores between pediatric resident levels. The Independent Variable was the education level of the resident (three levels, from 1 to 3 years). The three dependent variables were the average scores on the three constructs obtained from the questionnaire:

$\mathrm{a}=$ perceived pediatric resident educational training experiences (Medical Education)

$\mathrm{b}=$ perceived pediatric resident knowledge (Medical Knowledge).

$c=$ perceived pediatric resident competency for patient care (Professional Practice)

The purpose of the MANOVA was to determine if significant differences existed among the three groups of residents in the average levels of self-reported competence in the three constructs regarding communication disorders and augmentative and assistive technology. The software package SPSS version 15 Windows (SPSS, 2003) provided the computational analysis.

Assumptions of MANOVA. Before a MANOVA test can be used, data must meet certain assumptions, namely independent observations, homogeneity of variance, and normality of distribution (Tabachnick \& Fidell, 2001).

Several measures were used to improve control within the study. In this study, all observations were independent as each participant completed a single survey. Participants specified their years of resident training/ education. Residents participated in and received the same educational training within one university program, the University of Louisville's School of Medicine Pediatric 
residency program. All participants received the same survey. The survey was presented to all participants through the use of Survey Monkey (Survey Monkey, 2009). Finally, all survey information was coded to ensure participant privacy and ethical conduct in accordance to current HIPPA and IRB guidelines.

Multivariate Normal distribution of scores on the dependent variables were tested using histograms, see Appendix B. Each dependent variable demonstrated an acceptable level of normality across pediatric levels.

An assumption of the MANOVA is that the covariance matrices of the dependent variables are the same across groups (determined by levels of the independent variable) in the population. This is the multivariate analog of the assumption of equal variances for the ANOVA. Box's test for equality of covariance matrices investigates the differences in the variability between groups. According to Steven's (2002), if "normality has been achieved then Box's will not be significant" (p.278). In this study, Box's was not found significant (Box's $=.102, F(12,5573.158)=1.54, p>.05)$. Thus, homogeneity of variances was achieved within the study.

Main effect. The result of the MANOVA was a significant difference among the means of the dependent variables, the average levels of self-reported competence in the three constructs. Wilks' Lambda was used due to the presence of more than two groups formed by the independent variables. The main effect was significant, Wilks' Lambda $=.67, F(6,76)=2.789, p=.017<$ .05. $\eta^{2}$ was .18 , which was a large effect size according to Stevens (2002, p.197). 
Individual dependent variables. The effects for the individual dependent variables are illustrated in Table 13.

Table 13

Main Subject Effects for Education, Knowledge and Professional Practice

\begin{tabular}{cccccc}
$\begin{array}{l}\text { Dependent } \\
\text { Variables }\end{array}$ & $\begin{array}{l}\text { Sum of } \\
\text { Squares }\end{array}$ & $d f$ & $\begin{array}{l}\text { Mean } \\
\text { Square }\end{array}$ & $F$ & $p$ \\
\hline $\mathrm{E}$ & 8.58 & 2 & 4.30 & 6.43 & $.00^{*}$ \\
$\mathrm{~K}$ & 1.90 & 2 & .952 & 3.34 & $.046^{*}$ \\
$\mathrm{P}$ & .84 & 2 & .417 & 1.13 & .33 \\
\hline
\end{tabular}

Note. $\mathrm{E}=$ Education; $\mathrm{K}=$ Knowledge; $\mathrm{P}=$ Professional Practice ${ }^{*} \mathrm{p}<.05$

Several of the individual dependent variables were significant using a critical value of .05 . As can be seen in Table 13, Education was found to be significant, $F(2)=6.43, p=.00, p<.05$. For Education $\eta^{2}$ equaled .24 , a large effect size (Stevens, 2002).

Knowledge was also found to be significant, $F(2)=3.34, p=.046, p<.05$. For Knowledge $\eta^{2}$ equaled .14, a large effect size. Professional practice was not found significant, $F(2)=1.13, p=.33, p>.05$.

Post hoc. To follow up the effect, univariate ANOVA results were examined to make post hoc comparisons between variables and determine whether the interaction existed for each of the dependent variables. The descriptive statistics for the independent variables within each dependent variable are shown in Table 14 
Table 14

Between Subject Marginal Means for PLs across Education, Knowledge and Professional Practice

\begin{tabular}{|c|c|c|c|c|}
\hline \multicolumn{3}{|l|}{ Dependent } & \multirow{2}{*}{$\begin{array}{l}\text { Standard } \\
\text { Error }\end{array}$} & \multirow{2}{*}{$\begin{array}{l}\text { Standard } \\
\text { Deviation }\end{array}$} \\
\hline Variables & PL & Mean & & \\
\hline \multicolumn{5}{|l|}{$E$} \\
\hline & PL1 & 2.51 & .21 & .88 \\
\hline & PL2 & 2.16 & .25 & .31 \\
\hline & PL3 & 3.24 & .20 & .97 \\
\hline \multicolumn{5}{|l|}{ K } \\
\hline & PL1 & 3.33 & .14 & .57 \\
\hline & PL2 & 3.02 & .16 & .51 \\
\hline & PL3 & 3.55 & .13 & .51 \\
\hline \multicolumn{5}{|l|}{$\mathrm{P}$} \\
\hline & PL1 & 2.96 & .16 & .69 \\
\hline & PL2 & 2.94 & .18 & .38 \\
\hline & PL3 & 3.235 & .15 & .65 \\
\hline
\end{tabular}

Note. $\mathrm{E}=$ Education; $\mathrm{K}=$ Knowledge; $\mathrm{P}=$ Professional Practice

Note. PL1 = Pediatric Level 1; PL2 = Pediatric Level 2; PL3 = Pediatric Level 3

The comparisons between pediatric levels are shown in Table15. To obviate the inflation of Type I error rate due to repeated statistical tests, the Bonferroni correction was used for the multiple planned comparisons. 
Table 15

Bonferroni Multiple Comparisons across PLs for Education, Knowledge, and Professional Practice

\begin{tabular}{|c|c|c|c|c|}
\hline \multicolumn{5}{|l|}{ Dependent } \\
\hline Variables & PLs & MD & $S E$ & $p$ \\
\hline \multicolumn{5}{|l|}{$\bar{E}$} \\
\hline & PL1 X PL 2 & .34 & .32 & .89 \\
\hline & PL2 X PL 3 & -1.07 & .32 & $.01^{*}$ \\
\hline & PL3 X PL1 & $-.73^{*}$ & .29 & $.048^{*}$ \\
\hline \multicolumn{5}{|l|}{$\mathrm{K}$} \\
\hline & PL1 X PL 2 & .32 & .21 & .42 \\
\hline & PL2 X PL 3 & -.54 & .21 & $.04^{*}$ \\
\hline & PL3 X PL1 & -.22 & .19 & .78 \\
\hline \multicolumn{5}{|l|}{$P$} \\
\hline & PL1 X PL 2 & .02 & .24 & 1.00 \\
\hline & PL2 X PL 3 & -.30 & .23 & .65 \\
\hline & PL3 X PL1 & -.28 & .22 & .62 \\
\hline
\end{tabular}

Note. $\mathrm{E}=$ Education; $\mathrm{K}=$ Knowledge; $\mathrm{P}=$ Professional Practice

Note. PL1 = Pediatric Level 1; PL2 = Pediatric Level 2; PL3 = Pediatric Level 3 Note. F Ratios were derived from Wilks' lambda statistics ${ }^{*} p<.05$

Simple effects analyses were performed to examine the interaction between pediatric levels. This involved testing the difference between (a) PL 1 and PL 2 (b) PL 2 and PL 3 and (c) PL 1 and PL 3. 
Several comparisons were found to be statistically significant for interaction between independent variables using a critical value of .05 . Two comparisons were within Education, PL2 vs. PL $3(M=2.51$ vs. $M=3.24), p=$ $.01, p<.05$, and PL3 vs. PL1 $(M=3.24$ vs. $M=2.51), p=.048, p<.05$. One comparison was within Knowledge, PL2 vs. PL $3(M=3.02$ vs. $M=3.55), p=$ $.041, p<.05$. In all of the significant comparisons, the highest mean score was obtained by the respondents who were at pediatric level three (PL 3).

\section{Research Question 4: Independent $t$ - tests}

The fourth research question investigated the effects of demographic variables on residents' perceived competency across the three constructs. The five demographic variables include the following: gender, rotation completion, pediatric specialization educational methods, and educational time. Survey questions thirty-two to forty-five address these variables.

Independent $t$ tests and correlation coefficients were used to analyze this

data. The dependent variables were residents' perceived competency (the average scores on the three constructs obtained from the questionnaire) and the independent variables were demographic variables within the survey (gender, rotation completion, pediatric specialization educational methods, and educational time).

The software package SPSS version 15 Windows (SPSS, 2003), provided the computational analysis. All statistical analyses used .05 as the level of significance. However, to obviate the inflation of Type I error rate due to repeated statistical tests, the Bonferroni correction was used. It was 
implemented in the following manner. The three dependent variables were defined as a set. Within this set, the overall error rate was kept at .017 by dividing the number of tests into .05 and using the resulting value as the alpha level to be used for each comparison. Thus, the level of significance for all $t$ tests was $p=.017$.

Demographic variable gender. Males and females were compared on the average levels of self-reported competence in the three constructs regarding communication disorders and augmentative and assistive technology. Survey question thirty-two addressed this variable. For further information regarding the data for gender, see Appendix A.

Upon analysis of the $t$ test, a significant difference was not discovered between gender for any of the constructs: Medical Education, $t(41)=1.37, p=$ $.18, p>.05 ;$ Medical Knowledge, $t(41)=-.22, p=.83, p>.05$; and Professional Practice, $t(41)=1.45, p=.15, p>.05$. There was not a significant relationship between gender and self-reported competence across the three constructs.

Demographic variable pediatric specialization. Various specializations were coded as either Yes or No and the two groups were compared on the average levels of self-reported competence across the three constructs. Survey questions thirty-three addressed this variable. For further information regarding the data for specialization, see Appendix A.

A significant difference was not discovered between the two groups for any of the constructs: Medical Education, $t(41)=1.06, p=.29, p>.05$; Medical Knowledge, $t(41)=1.53, p=.13, p>.05$; and Professional Practice, $t(41)=$ 
$1.52, p=.14, p>.05$. There was not a significant relationship between completion of a specialization and self-reported competence across the three constructs.

Demographic variable rotation completion. The $t-$ tests regarding rotation completion compared how pediatric residents perceived their average levels of self-reported competence in the three constructs regarding communication disorders and augmentative and assistive technology upon completion or non-completion of a specific rotation. Participants were coded as either 'Yes' or 'No' and the two groups will be compared on the average levels of self-reported competence in the three constructs regarding communication disorders and augmentative and assistive technology. Survey questions thirtyfive addressed these variables.

Separate data was analyzed for the pediatric resident rotations deemed relevant to communication disorders and AAC. These included the following: adolescent medicine, ambulatory pediatrics, child development, community health programs, genetics, in- patient (wards), pediatric ICU, private practitioner's office and Weisskopf Children Evaluation Center (WCEC). For a complete listing of pediatric resident rotation changes across PLs see Table 6.

The ability of the data to meet methodological assumptions was independently addressed for each rotation. The assumptions for an independent $t$ test included independence, normality, and equality of variances.

Table 16 provides a summary of the rotations found significant. For further information regarding the data for rotation completion, see Appendix C. 
Table 16

Summary of Rotation $\mathrm{t}$ - tests $(\mathrm{N}=43)$

IV DV $\quad$ Yes No $t$ (df) $\quad p$

\begin{tabular}{llllll}
\hline AMR & E & 2.93 & 2.20 & $t(41)=-2.55$ & $p=.015^{\star \star}$ \\
PICU & E & 3.31 & 2.35 & $t(41)=-3.84$ & $p=.00^{\star \star}$ \\
WCEC & E & 4.08 & 2.53 & $t(41)=-4.21$ & $p=.00^{\star \star}$
\end{tabular}

Note. $\mathrm{E}=$ Medical Education; $\mathrm{K}=$ Knowledge; $\mathrm{P}=$ Professional Practice Note. $A M R=$ Adolescent Medicine Rotation, $P I C U=$ Pediatric Intensive Care Unit, $W C E C=$ Weisskopf Child Evaluation Center Note. ${ }^{* *} p<.017$

Demographic variable: Educational methods. Another demographic variable analyzed within the data was educational methods. The independent variable, educational methods were coded into two groups, 'Yes' or 'No'. The two groups (Yes or No) were compared for mean differences on the average levels of self-reported competence in the three constructs regarding communication disorders and augmentative and assistive technology. Survey questions $35,36,38$ and 39 addressed these variables. The initial question posed was directed to the occurrence of any educational experiences regarding communication disorders or AAC within a rotation. The rest of the analysis that follows addresses specific formats were these learning experiences may have occurred. Educational methods will include one or more of the following: ambulatory pediatric rotation, subspecialty rotation, morning report, core conference, didactic or board conference. 
Table 17 provides a summary of the educational methods that were found significant. For further information regarding the data for educational methods, see Appendix C.

Table 17

Summary of Didactic $t$ - tests $(N=43)$

DV Mean (Yes) Mean (No) $t$ (df)

$p$

$E: C D$

3.51

2.55

$t(41)=-2.74$

$p=.009^{\star \star}$

$P P: C D$

3.68

2.94

$t(41)=-3.25$

$p=.002^{\star \star}$

Note. $\mathrm{DV}=$ Dependent Variable

Note. $\mathrm{E}=$ Medical Education; $\mathrm{K}=$ Knowledge; $\mathrm{P}=$ Professional Practice

Note. $\mathrm{CD}=$ Communication Disorder

Note. ${ }^{\star \star} p<.017$

\section{Demographic variable: Educational time}

Survey questions 37 and 40 addressed these variables. Educational time was the only measure that was not coded. The average of the sum total was used. It was used as one of the variables in a set of correlation coefficients. For correlation coefficients, a linear relationship between two variables being correlated was assumed.

A frequency distribution categorizing the range of educational time spent on the topics of communication disorders and AAC was previously presented in Table 7. The range of the distribution was from less than one hour to more than four hours. Most of responders $(10 / 17,58.8 \%)$ indicated that they received one to two hours of instruction on communication disorders, and most of responders 
for $\operatorname{AAC}(7 / 11,63.7 \%)$ indicated that they also received one to two hours of instruction time regarding AAC.

A correlation study was then conducted to investigate the relationship between education time for communication disorders and the three constructs used within the study. These were noted as follows in Table 18:

Table 18

Correlations Between $C D$ Hours and Constructs $(N=17)$

\begin{tabular}{lcccc} 
Variable & HCD & MED & MK & PP \\
\hline HCD & $-\cdots$ & .20 & .21 & .48 \\
MED & & - & $.59^{\star \star}$ & $.67^{\star \star}$ \\
K & & & $-\cdots$ & $.68^{\star \star}$ \\
PP & & & &...-- \\
\hline
\end{tabular}

Note. $\mathrm{HCD}=$ Hours Communication Disorders; MED = Medical Education; $\mathrm{MK}=$ Medical Knowledge, and PP = Professional Practice Note. ${ }^{\star} p<.05 .{ }^{\star \star} p<.01$ (two tailed).

As can be seen from the above, there was not a significant relationship between the hours of education time and perceive competency across the three constructs. The results of the rest of the correlation analysis replicate the previous findings in Table 11. See Table 11's analysis for further information regarding the significant relationships between Medical Education, Medical Knowledge and Professional Practice.

A correlation study was also conducted to investigate the relationship between educational time for AAC and the three constructs used within the study. These were noted in Table 19. 
Table 19

Correlations Between AAC Hours and Constructs $(N=1)$

Variable HAAC MED MK PP

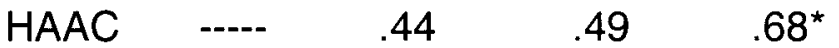

MED $\quad-\quad .59^{\star \star} \quad .67^{\star \star}$

MK

NP

Knowledge, and PP = Professional Practice

Note. ${ }^{*} p<.05 .{ }^{* *} p<.01$ (two tailed).

As can be seen from the above, there was not a significant relationship between the hours of education time and perceive competency across two of the three constructs, Medical Education and Medical Knowledge. There was a significant relationship between hours of education time and Professional Practice. They were moderately correlated, $r(11)=.667, p<.05$.

The results of the rest of the correlation analysis replicate the previous findings in Table 11. See Table 11's analysis for further information regarding the significant relationships between Medical Education, Medical Knowledge and Professional Practice.

\section{Research Question 5: Informal Qualitative}

The fifth question investigated the perspectives of pediatric residents regarding communication disorders and $A A C$ as part of their training. This question provided qualitative data gathered through responses to several openended questions. The qualitative aspects of Question 5 provided more insight regarding the perspectives and experiences of pediatric residents within their 
educational experiences. Information for this qualitative section was gathered from questions 41 to 43 on the survey and from the informal focus group. For information regarding the sample population, procedures, and data collection methods, see Data Selection and Coding.

Conversational subjects covered the research questions posed by the investigator. The topics included the following: knowledge and experience with communication disorders and AAC, education, and a pediatrician's role. All members of informal focus group were engaged during the discussion and presented their thoughts and opinions.

\section{Knowledge of Communication Disorders and AAC}

"How familiar are you with communication disorders and AAC?" The first resident to respond stated that he was more familiar with AAC within the adult population due to experience with laryngectomies and head-neck cancers. He wasn't as familiar with AAC for the pediatric population. The resident went on to say that he had more familiarity with communication disorders and $A A C$ as being within the context of educational systems. 'I'm more familiar with kids getting worked with at school than on an outpatient basis. ...doing to speech at school, er, once a week or whatever for various issues.' Other participants joined in the discussion to add that they too were familiar with 'speech' being provided in school, (kids get) 'like special reading classes and speech therapy. All that kind of stuff school provided.'

Residents were specifically asked about their knowledge of AAC. One resident referred his knowledge to a book he had read where the main character 
had 'locked in syndrome'. 'He had a speech therapist work with him so that he could communicate...one letter at a time.' Another resident had worked with a child that used an iPad as voice output communication device. She stated that

"He had a program on the iPad that helped him tell what he wants, you know, like, I want to drink, and the iPad would talk. Or like I want milk, I want to watch TV or whatever. It was mild to moderate mental retardation with a pervasive developmental disorder. I mean, he verbalized, you know. You could ask him yes or no questions, and he would respond. The parents obviously understood him a lot better than I did. We held the conversation and stuff without the use of the device, but the parents were showing how he used and stuff at school." (personal communication)

\section{Experience with Communication Disorders and AAC}

The residents were asked about their experience with speech therapy and AAC within their work settings. The residents knew that had speech therapists in the hospital, and that they conducted swallow studies. A resident stated that 'I think that (swallow study) is the large part of our experience with speech.' The same resident described brief contact with speech pathologists within the neonatal intensive care unit. 'I know we have speech. I just know the one really nice speech girl. She has dark brown hair.... I only know her because I met her in the NICU once.' Another resident stated that they were not always sure 'where to find them' (physical therapists, occupational therapists, etc.).

The residents stated that much of the educational focus is in-patient. They therefore do not often address communication disorders and AAC. One resident stated,

"In an inpatient setting if the patient has a lisp or something else, if it is not a very acute issue this is not something speech helps us address in an inpatient setting. Because they are not being admitted because, because they have a lisp. You know, there is a bigger something going on. I think 
a lot of times that issue is dealt with as an outpatient."(personal communication)

Another resident added,

"A lot of our early training is very inpatient heavy, so you don't get a lot of kids that are getting admitted for those things (communication disorders and AAC). They might have them, like your (pointed to other resident) patient with the iPad, but more of the outpatient level we don't get a lot of exposure to it quite yet." (personal communication)

\section{Survey}

The first qualitative question was posed with the query, 'What are your thoughts about the survey regarding communication disorders and AAC?' Of the three residents attending at that time, one response was 'Fine' and 'Thorough,' and the other resident stated that it looked long and she looked at it but did not complete it. The other residents stated that they had not completed it.

Education. The residents were asked, 'What are your thoughts about your educational training for communication disorders and AAC?' The residents stated that they relied on Weisskopf Center to provide them information regarding communication disorders and AAC. The first resident to respond stated that 'Not having done CEC, I'm counting on that as being my exposure, I guess.' Another resident added that 'we don't get it until our third year... at the Weisskopf Center, and so we don't get a lot of ...so we don't get a lot of experience of it. We just know that kind of (unintelligible) thing to the Weisskopf center that you send kids over there and they help them.'

A resident speculated about other's attitudes about communication disorders and AAC. She stated, 
"The problem is primary care people that want to do primary care. I think want a little different stuff than the people who want to specialize and I see a lot of my colleagues being, like, 'This is dumb, why do I need to sit and watch as well as study? Why do I need to...' Know what I mean? Because they are very focused, and that is who I think want a broader perspective would enjoy that kind of stuff." (personal communication)

Roles. The second and third qualitative queries were asked with the question, What are your current and future roles regarding communication disorders and AAC? With regard to communication disorders, AAC and general Developmental Disabilities, the residents felt their first role was to refer these children to a specialist. In the words of one resident, 'We're an organizer, we move people to the appropriate people. We don't do much treatment.' One of the residents went on to provide an example and clarify her perceived role.

"If you were to have a, you know, murmur. I identify the murmur. I know it is there, but I am not the one to help treat and follow it. Off to cardiology you go. So it is kind of like our role is, especially the general pediatrician, which I think most of us sitting right here is identifying the problem and then referring. I think that is the most difficult because you ask the parents, like, do you understand what Johnny says? (parents) Oh yeah, I got it, and you are like okay well then is it me? Am I just not getting it? You know, like, at what point is there a problem with Johnny or you know, and the family is ignoring this issue." (personal communication)

The residents were asked, 'How comfortable do you feel with then guiding the parents to identification of a problem?' In response to this question, the residents began to talk about referring individuals to services. One of the residents stated that they do not really learn about referral sources until their third year.

"I think you hear a lot from people that do their community or CEC months in their third year and they say 'Wow, I didn't even know these resources existed.' I spent three years of treating kids here and there and I didn't know that I could send somebody to be evaluated for this." (personal communication) 
In response to this comment, another resident stated,

"That is a problem that they are trying to work on. CEC only allows so many residents at a time to be there and they have to fill their time to get it so it gets pushed (unintelligible)." (personal communication)

The same resident went on to discuss possible solutions to problems with

referrals. This included using a resource book available in some medical

education facilities.

"One of the attendings (omitted for privacy) we have came from another place, and she thought it was kind of strange when she came here that nobody gave her resource book. And I was like, what is a resource book? And she said, when we started residency, cause a lot of people move from out of town. You know? I do not know Louisville from (omitted for privacy) from anywhere else that I am from, so you do not know the (unintelligible) and you do not know the community, but nevertheless, it is very important aspect when you are a pediatrician. You have to know these things, so they called it a resource book, and it was like you identified that Johnny has, you know, whatever problem, you can look it up, and these are your options for PT. The patient has Medicaid, this is your option. If they have insurance, these are some other options, and these are your local orthopedists, or these are your local whatever it may be. She was like I cannot believe you guys do not have one of those, and I looked at her said, I do not know. You know, it is just kind of stuff you figure out. I mean, we will identify (unintelligible) and you will come out, and you will be like wow, this kid has $\mathrm{X}, \mathrm{Y}$, or $\mathrm{Z}$, and you are like, I do not know where to send them because I do not know what we have here, and that is when the attendings are like oh we can send them over here, we can send them over there, and that kind of stuff." (personal communication)

The same resident went on to state,

"Knowing where the information is, it is half the battle. You know what I mean? (unintelligible) but I just like to know more, but I feel like I can identify a problem. I may not be able to diagnosis it, but I could identify it and find a resource, and get you help, and so I guess I am halfway there." (personal communication)

Several residents mentioned concerns regarding their ability to provide referral information in the future. 
"What is happening is like I know what to do with them, you know what I mean? But I am in a population where I have resources. What happens when I am in middle of nowhere Kentucky. Obviously, I am not going to be the one offering the speech therapy. I definitely do not think that you know, but it maybe my role should be asked to be a little bit more knowledgeable. Maybe about what might be going on. There is a type of broad understanding of you know, it could be $\mathrm{X}, \mathrm{Y}$, or $\mathrm{Z}$. I think it is probably $X$. We are going to send you to (unintelligible). You know, like, that kind of a thing as opposed to being absolutely clueless and saying yeah you have a speech problem." (personal communication)

The residents also discussed following up on previous referrals and ongoing services as part of their role.

"It is like once that problem is diagnosed, it is being managed by someone else unless they come in and say I do not like that speech person, or I am not seeing any improvement, or my referral ran out, write me another one, you are not, you know? And you know, you check the chart and you see that you know you get the letter back from whoever this is. You know, we are working with him on this, and we are progressing with this, and this is looking good. And you go okay great." (personal communication)

\section{Summary}

\section{Quantitative Data}

Primary research question. Within the quantitative results of this data analysis, several outcomes found significant differences. Beginning with the main question, 'Is there a significant difference in pediatric resident's perceived competency over their three years of educational training?' was answered. Significant difference was found within the main effect of the MANOVA, Wilks' Lambda $=.672, F(6,76)=2.789, p=.017<.05$, with a large effect size, $\eta^{2}=$ .180. When identifying the source of that significance, two out of three of the individual dependent variables were significant. Education was found to be significant, $F(2)=6.43, p=.004, p<.05$, with a large effect size, $\eta^{2}=.243$. 
Knowledge was also found to be significant, $F(2)=3.34, p=.046, p<.05$, with another large effect size, $\eta^{2}$ equaled .143 .

Simple effects analyses were performed to examine the interaction between pediatric levels, testing the difference between (a) PL 1 and PL 2 (b) PL 2 and PL 3 and (c) PL 1 and PL 3. Three comparisons were significant. Two comparisons were within Medical Education, $\mathrm{PL2}$ vs. $\mathrm{PL} 3(\mathrm{M}=2.507$ vs. $\mathrm{M}=$ $3.235), p=.005, p<.05$, and PL3 vs. PL1 ( $M=3.235$ vs. $M=2.507), p=.048, p$ $<.05$. One comparison was within Knowledge, $P L 2$ vs. $P L 3$ ( $M=3.015$ vs. $M=$ 3.549), $p=.041, p<.05$.

Demographic variables. The forth hypothesis was then addressed, the effects of demographic variables on residents' perceived competency across the three constructs. The five demographic variables investigated were: gender, rotation completion, pediatric specialization, educational methods, and educational time. Gender and specialization were not found to present any significant differences.

Rotations. Within rotation completion, four variables were found to be significant. These included adolescent medicine rotation, ambulatory pediatrics, pediatric intensive care unit, and WCEC. For adolescent medicine, Medical Education was found to be significant. Medical Education, $t(41)=-2.547, p=$ $.015, p<.05$. Within the analysis for ambulatory pediatrics rotation, a significant difference was found for Medical Education, $t(41)=-2.196, p=.034, p<.05$. For the Pediatric Intensive Care Unit a significant difference was found between two constructs, Medical Education and Medical Knowledge, Medical Education, $t$ (41) 
$=-3.841, p=.000, p<.05 ;$ Medical Knowledge, $t(41)=-2.320, p=.025, p<.05$. Finally, for WCEC a significant difference was found between completion of the rotation and self-reported competence for Medical Education, $t(41)=-4.213, F=$ $.000, p<.05$

Educational methods. The next demographic variable to be investigated was educational methods. Confounding factors inhibited the completion of much of this analysis. The major factor was limited data. All information except for one data set was able to completed for educational methods and communication disorders, but information regarding AAC could only be analyzed for the data within Rotation. All other data analysis was confounded by a minimal sample size.

In review of the data analyzed, significant differences were found between the within the following educational experiences: rotations, ambulatory pediatric rotation and Didactics. For rotations, a significant relationship was found between rotation and perceived levels of competency for Medical Education within both communication disorders and AAC. For communication disorders: Medical Education, $t(41)=-2.401, p=.021, p<.05$; For AAC: Medical Education, $t(41)=-2.369, p=.021, p<.05$. Regarding ambulatory pediatric rotation, there was a significant relationship between both Medical Education and Medical Knowledge and the presentation of educational experiences regarding communication disorders: Medical Education, $t(41)=-2.326, p=.025, p<.05$; Medical Knowledge, $t(41)=-2189, p=.034, p<.05$.

A significant relationship was found between Medical Education, Medical 
Knowledge and Professional Practice and the presentation of educational experiences regarding communication disorders within Didactics. Medical Education, $t(41)=-2.735, p=.009, p<05$; Medical Knowledge, $t(41)=-2.146$, $p=.038, p<.05 ;$ and Professional Practice, $t(41)=-3.252, p=.002, p<.05$.

Educational time. The relationship between the three constructs and educational time was investigated. There was not a significant relationship between the hours of education time and perceive competency across the three constructs for communication disorders. For AAC there was a moderate, significant relationship between hours of education time and Professional Practice, $r(11)=.667, p<.05$.

\section{Qualitative Data}

The qualitative data added depth to the quantitative information. The qualitative information was gathered during an interview-style focus group. The informal data collected did provide some insights regarding pediatric resident's thoughts towards their knowledge, experience, education and their roles as pediatricians regarding communication disorders and AAC. In general, the residents expressed some knowledge and experience with the realm of communication disorders and AAC, but to a significantly limited degree. Reasons for this lack were cited as being secondary to working within an inpatient versus out-patient setting, and non-completion of the WCEC rotation. With regard to their current and future roles, pediatric residents felt that these included having knowledge of the various sources for referrals, providing 
referrals, and following-up on referrals. Gathering or providing referral information was also voiced as an ongoing issue. 


\section{CHAPTER V}

\section{DISCUSSION}

The purpose of this study was to identify possible differences across pediatric resident levels regarding competence within three constructs with a specific focus on communication disorders and AAC. This chapter provides an overview of the purpose and procedures used in this investigation. Following this, conclusions related to each research question are described. Limitations of the investigation are then presented followed by the implications of this study. Finally, the chapter concludes with recommendations for future research.

\section{Overview}

Communication is key feature to every aspect of life. It has been demonstrated that children with general communication impairments as well as complex communication needs rely on pediatricians to prescribe the services of speech language pathologists. In light of the continuing and increasing need, it is therefore important to ascertain whether medical residents are receiving the necessary training in their educational program to fulfill their role. Within the framework of the current ACGME competencies, it is important to understand how pediatric residents perceive their current level of abilities 


\section{Research Questions}

This study investigated differences in perceived ACGME competency regarding communication disorders and AAC across the three levels of pediatric residency at the University of Louisville. Discussion regarding the findings of each research question is presented in this section.

Research question 1. To what extent are there differences in perceived pediatric resident educational training experiences for communication disorders and AAC across pediatric levels? The data provided evidence of some differences between pediatric levels for Medical Education. Differences in perceptions were found regarding educational training experiences for both communication disorders and AAC.

Statistical significance for Medical Education continued within the paired comparisons of Pediatric Levels. The main differences were found between levels one and three, and level two and three. The only level that consistently appeared within the data was level three. Further investigation regarding the difference between the third level of pediatric residency and the prior levels of residency might be warranted to better understand this observation.

If there are some significant differences between the means for the construct of Medical Education across pediatric levels, then the expected outcome is increased educational experiences regarding communication disorders and AAC over time. This assumption does not necessarily hold true.

Although significant differences were found between the main effect and paired comparisons, the mean of survey responses for the constructs of medical 
education tended toward a neutral. Within a Likert scale of one to five, with one being strongly disagree, and five being strongly agree, the composite mean for Medical Education was 2.71 ( 2 being disagree and 3 being neutral). The average response for Medical Education competency statements was therefore disagreement to neutral. This mean score does not provide definitive information regarding pediatric resident's perceptions of their competency on the whole. All the same, there is a trend towards disagreement with the construct questions. Further examination of residents' perceived competency was gained by inspecting their responses to specific survey items within the construct of Medical Education. Within the construct Medical Education, survey questions one through five were reviewed. It appears that most of the current residents do not view themselves as having participated in educational experiences specifically for communication disorders or AAC. It was found that the majority of residents (60.4\%) disagreed or strongly disagreed with the question, "I participated in a block rotation for experience in behavioral/ developmental pediatrics.' Regarding the question, 'I have had practiced-based learning regarding communication disorders,' residents strongly disagreed or disagreed $41.9 \%$ of the time, and $34.9 \%$ agreed or strongly agreed. For the same question directed towards AAC, residents' responses were more negative. Over half (51.2\%) strongly disagreed or disagreed. When asked about educational training for the management of a child with a communication disorder, $41.8 \%$ disagreed or strongly disagreed, while $30.2 \%$ agreed. When the same question was again asked regarding AAC, 
the majority of responses were again negative. Over half $(58.1 \%)$ disagreed or strongly disagreed with the statement.

Thus, although there are significant differences across pediatric levels for the perception of educational experiences, this may not indicate a positive result. The overall trend appears to be that the majority of residents do not believe they are receiving educational experiences regarding communication disorders and especially AAC. Which educational format did not appear to make much of differences in residences' responses. For block rotation, practice-based learning, and training for management of a child, the trend was consistently the same.

Research question 2. Is there a significant difference in perceived pediatric resident knowledge of communication disorders and AAC across pediatric levels? The data provided evidence of some differences between pediatric levels for the constructs Medical Knowledge. In this study differences were found in perceptions regarding knowledge for both communication disorders and AAC were found across the pediatric levels.

Statistical significance within the construct of Medical Knowledge continued within the paired comparisons of Pediatric Levels. One of the three comparisons was significant; the difference between levels two and three.

For this question, residents responded in an inconsistent manner. Residents appear confident in some specific abilities within this construct. Yet, one particular area had a trend towards a possible area of weakness.

Before investigating the specific construct questions, the overall mean needs discussion. The composite mean for Medical Knowledge was 3.34 ( 3 
being neutral and 4 being agree). In looking at this score, residents neither agreed nor disagreed with competency statements for Medical Knowledge. Again this mean does not provide definitive data regarding residents' perceptions of their competency. The cause of the trend may either be a lack of a strong response, the inconsistency in residents' responses within the construct or a lack of surety in residents' abilities.

In a similar fashion, detailed investigation of the survey items within the construct Medical Knowledge supplies insight on trends within the pediatric resident population. This construct consisted of six survey questions, specifically survey items six through eleven. Within this construct, residents expressed both confidence and uncertainty regarding their competence. The first survey item for this construct addressed the identification of communication disorders. Most residents $(74.4 \%)$ agreed or strongly agreed with the statement, "I can recognize abnormal communication development." Similar findings were observed with the next item, "I can recognize of abnormal speech development." Again, most residents agreed or strongly agreed $(81.4 \%)$.

Upon examination of the impact of a communication disorder or AAC device on developmental patterns and education success, residents reported more confidence. Most residents (83.8\%) agreed or strongly agreed with the statement, "I understand the impact of a communication disorder on a child's development pattern and educational success." When the question addressed AAC, most residents agreed or strongly agreed (58.2\%). 
Interestingly, when the topic shifted to care coordination, residents responded in a less confident manner. Most residents (44.2\%) disagreed or strongly disagree with the statement, "I have knowledge of communication disorders as a care coordinator." An even greater majority disagreed or strongly disagreed with that statement when it was directed to AAC (58.2\%). Care coordination is a vital part of the responsibilities of pediatricians. It is one of the current and basic AAP policies guiding the treatment and care of patients. The implications of a negative trend within this area are significant and will be further discussed later within the implications portion of this chapter.

Research question 3. Is there a significant difference in perceived pediatric resident competency for professional practice regarding the care of children with communication disorders and AAC across pediatric levels?

It was remarkable that there was no difference between the means of the pediatric resident levels for the construct of Professional Practice. This is particularly interesting seeing that this construct had the most survey questions, items 12 to 31 . One would also expect that change would be evident due to the number skills expected within this construct. This construct incorporates five of the six ACGME competencies within its definition. All the same, residents did not demonstrate any significant changes in their perceived competencies over the course of their pediatric training. Their responses did not present any significant agreement or disagreement or definitive data towards their competency. Their responses had a trend toward neutral with an overall mean of 3.07. The trend toward neutrality and a lack of significance could indicate several things. The 
cause of the trend may again be either a lack of a strong response, or a lack of surety in residents' abilities.

The other possibility for the trend towards neutrality may be in question construction. There was a high correlation between this items of the construct (Cronbach's alpha was .94), so the trend was not inconsistency between the construct items. In ruling out item intercorrelation, there is need for further analysis of question wording and semantics for further use of this survey.

Research question 4. What effect do demographic variables influence resident's perceived competency? The fourth research question addressed the various demographic variables that were presented within the survey. These variables provided additional data regarding pediatric resident population of today. Demographic items included the following: gender, specialization, rotation completion, educational methods and educational time.

Independent variable gender. Males and females were compared on the average levels of self-reported competence in the three constructs regarding communication disorders and augmentative and assistive technology. It is a positive outcome that there were no differences between genders regarding their perceptions of their competency. It is interesting that more females responded than males. This corresponds with the trend today for an increase in women within the field of pediatrics (Goodman, 2005).

Independent variable, pediatric specialization. Various specializations were coded as either Yes or No and the two groups will be compared on the average levels of self-reported competence in the three constructs regarding 
communication disorders and augmentative and assistive technology. The data did not find any significant differences between the perceptions of individuals pursuing a specialization versus those that were not. One might speculate that the lack of difference might be due to other dynamics. Further exploration of this could provide a better understanding of these possibilities.

This exploration needs to take into consideration the whole residency process. The core pediatric residency training consists of three years, and often specialization requires a significant amount of additional education. This most often consists of a three-year fellowship. The lack of difference between scores (specialization versus none) indicates that additional education does not change perceptions of competency.

On one hand this is positive. Specialization or not, the American Academy of Pediatrics expects all pediatricians to have knowledge of communication disorders and AAC (Desch, et al., 2008). Yet, for the constructs that had a negative trend, such as Medical Education and Medical Knowledge, this is concerning. The core educational experience must then undergo further investigation regarding its content for communication disorders and AAC. Changes within the core training may be needed.

Independent variable, rotation completion. Participants were coded as either Yes or No and the two groups will be compared on the average levels of self-reported competence in the three constructs regarding communication disorders and augmentative and assistive technology. This variable was pertinent for a number of reasons. Rotations are completed in sequence within 
the training levels of pediatric levels and may impact perceived competency. Rotations also provide a wide array of experience with some providing more information about developmental disabilities than others. Identification of which rotations change perceptions of competency is then important to both this study as well as the educational services at the University of Louisville.

Of the rotations that were identified as relative to this study for further analysis, three demonstrated a significant difference within residents' perceived competency. A significant difference was found between the means for completion versus non-completion of rotations for adolescent medicine, pediatric intensive care unit and Weisskopf Child Evaluation Center (WCEC).

When looking at Table 6 , rotation participation over pediatric levels, the differences are apparent, especially for the pediatric intensive care unit and WCEC. With these two rotations, residents did not participate until their final pediatric level (third year). For the Pediatric Intensive Care Unit, one resident participated in their second level, and $15(88.2 \%)$ participated in the third year. For Weisskopf Child Evaluation Center, no residents reported attending until their third year with five total $(29.4 \%)$. Therefore, it appears the difference could be due to resident training levels.

However, upon through analysis it was found that adolescent medicine appears to be an exception. This rotation has eight participants within both first and second levels. The third level participation also had an increase in attendance, but the change in percentage is not dramatic. Completion shifts from eight participants $(72.7 \%)$ to $14(82.4 \%)$. 
Other evidence against the theory of rotation participation as having an effect on competency can be seen in the data regarding critical care medicine and neurology (see Table 6). Both areas demonstrated a significant increase in participation over their pediatric levels, but neither one found significant differences for completion versus non-completion for perceived competence across the three constructs. Further investigation is needed to identify what aspects of rotations contribute to differences in perceived competency for communication disorders and AAC.

If the timing of the rotation is not a factor, then further analysis is needed regarding educational means. In review of previously reported demographic information, it was notable that the majority of residents $(60.5 \%)$ reported not discussing communication disorders within a rotation, and $74.4 \%$ reported not discussing AAC within a rotation. Several questions arise from this information. If rotations are not addressing these topics, do they need to be? Is this information more important within the context of some rotations versus others? Finally, if the timing of the significant rotations changed, would that change statistical outcomes in future studies?

Other demographic data for educational methods was regarding time. For those that did receive some educational instruction regarding communication disorders, the typical instructional time was from one to two hours. For those that received some education regarding $A A C$, the typical instruction time was an hour. Again this information needs further assessment. Is this amount of educational time adequate? What types of educational methods are being 
applied within this time frame, and what is most effective?

With pediatric rotations being one of the main forms of education within the program, the majority do not appear to be providing educational experiences for communication disorders and AAC. Further examination of this topic is needed specifically focusing on the timing of rotations, the educational focus of each rotation and how information regarding communication disorders and AAC is disseminated within the current hour to two hours of educational time.

Independent variable, educational methods. This demographic variable was coded into two groups and analyzed for the average levels of selfreported competence in the three constructs regarding communication disorders and augmentative and assistive technology. Educational methods included one or more of the following: ambulatory pediatric rotation, subspecialty rotation, morning report, core conference, didactic or board conference. Of the methods presented, the only one found significant was didactics.

The use of didactics was found to have a significant difference in perceived competency for Medical Education and Professional Practice. Specific educational methods and content within each rotation were not analyzed in detail for the purpose of this study. Some of the variation within the data may be due to similarities or differences the educational format within these rotations. As noted before, the various educational methods using within residency training needs further analysis.

Independent variable, educational time. Educational time was the amount of time a pediatric resident received instruction through the above 
educational methods. The variable educational time was summed for each participant and correlated with the average levels of self-reported competence in the three constructs regarding communication disorders and augmentative and assistive technology.

One such variation within rotations or educational methods could be allotted time for different topics. A significant relationship was not found between educational time regarding communication disorders and perceived competency within the constructs. For AAC there was a moderate, significant relationship between hours of education time and Professional Practice, but not for Medical Knowledge or Education.

This finding is not surprising in light of the educational time reported by residents. As noted previously, residents receiving some educational instruction regarding communication disorders typically reported one to two hours of instructional time. For those that received some education regarding $\mathrm{AAC}$, the typical instruction time was an hour. If the amount of educational time is limited, then its impact on perceived competencies may also be limited. Further analysis of this relationship is needed.

Research question 5. The fifth question investigated the perspectives of pediatric residents regarding communication disorders and $A A C$ as part of their training. This question provided qualitative data gathered through responses to several open-ended questions. The qualitative aspects of Question 5 provided more insight regarding the perspectives and experiences of pediatric residents within their educational experiences. 
A lack of educational emphasis or training was a topic of discussion from the residents within the informal focus group. Within this meeting, residents expressed some knowledge and experience with the realm of communication disorders and $A A C$, but to a significantly limited degree. One reasons for this may be due to working within an in-patient versus out-patient setting. Regarding the inpatient treatment, one resident stated,

"A lot of our early training is very inpatient heavy, so you don't get a lot of kids that are getting admitted for those things (communication disorders and $A A C$ ). They might have them, like your (pointed to other resident) patient with the iPad, but more of the outpatient level we don't get a lot of exposure to it quite yet." (personal communication)

This qualitative information corresponds with the quantitative, specifically, the trend towards a lack in educational experiences. As noted within research question one, the majority of residents do not believe they are receiving educational experiences regarding communication disorders and especially AAC.

The resident's statements also support the data regarding educational time. Residents do not "get a lot of exposure". Typically one to two hours of educational time is spent on communication disorders and AAC.

Another reason for this lack of education was reported as secondary to non-completion of the Weisskopf Child Evaluation Center rotation. One resident stated, "Not having done CEC, I'm counting on that as being my exposure, I guess." Another resident added that...

"we don't get it until our third year... at the Weisskopf Center, and so we don't get a lot of ...so we don't get a lot of experience of it. We just know that kind of [unintelligible] thing to the Weisskopf Center that you send kids over there and they help them." (personal communication) 
These comments again support the findings within the quantitative data. Significant differences were found in completion versus non-completion of Weisskopf Child Evaluation Center rotation and resident's perceptions of competency. At the same time, adolescent medicine and the pediatric intensive care unit were also found significant. It is curious that they were not mentioned by name by the residents within the focus group. What are the differences between the Weisskopf rotation and adolescent medicine and the pediatric intensive care unit rotations?

Further investigation of the Weisskopf Child Evaluation rotation is needed to not only identify why residents "rely" on it for these topics, but also to investigate the timing of this rotation. This rotation is identified as occurring within the third pediatric level. In resident's words, "we don't get it until the third year." The question is then how perceptions of competency would change across pediatric levels if the timing of this rotation changed?

The qualitative information provided by the residents reinforced the quantitative findings. The residents were confident regarding their current and future roles when identifying a need, providing referrals, and following-up on referrals. One resident labeled her role as that of an "organizer". At the same time, provision of referral information for specific specialists was identified as a potential issue. One of the residents stated that they do not really learn about referral sources until their third year.

"I think you hear a lot from people that do their community or CEC months in their third year and they say 'Wow, I didn't even know these resources existed.' I spent three years of treating kids here and there and I didn't 
know that I could send somebody to be evaluated for this."(personal communication)

In response to this comment, another resident stated,

"That is a problem that they are trying to work on. CEC only allows so many residents at a time to be there and they have to fill their time to get it so it gets pushed (unintelligible)." (personal communication)

Again the resident's introduced the topic of Weisskopf Center, and this time within the context of referrals. The timing of this rotation may impact not only resident's perceptions of competency, but also their ability to make referrals.

One resident stated that this rotations timing is "a problem they are trying to work on." These comments are cause, again, for further investigation of this rotation and its impact. Specific analysis of the potential effects or barriers for any potential change is warranted.

\section{Research Comparison}

When comparing the results of this study to that of similar investigations of this population, both similarities and differences can be found. Previous research found a lack of confidence in prescribing therapies or devices. According to Sneed et al.'s (2004), a little more than half of the pediatricians (52.2\%) surveyed stated that they would recommend professional services or therapy. The results of this study demonstrate a step forward regarding resident's willingness to provide a referral for services. Sneed et al. (2004) also found little knowledge base of residents regarding CSHCN. Within this study, the majority of residents again stated that they felt competent in their ability to identify a communication disorders and need for AAC. 
Previous research found a significant need for training (Sneed, et al., 2000). Training was likewise identified as a continuing need in this study. The amount of time spent for educational instruction about communication disorders and AAC appear to have not changed. Sneed, et al. (2000) found only $5 \%$ of his surveyed population received greater than 1 hour of training in any category of medical equipment (including AAC). The typical instruction time within this study for those that had received some education regarding AAC $(24.6 \%$ of the population) was found to be an hour. Sneed's findings for AAC education still hold true, as there is "a striking sense of inadequate training evidenced among residents ... for the various DME categories" (p.559).

When reviewing the overall educational experiences, Sneed et al. (2000) previously found only $19 \%$ of respondents felt they had adequate training regarding communication disorders. Again, this result is similar to the findings within this study. As noted previously in resident's responses, most reported a lack of educational experience with behavioral/ developmental pediatrics, practiced-based learning regarding communication disorders and AAC, management of a child with a communication disorder or AAC. The majority (60.5\%) reported not discussing communication disorders within a rotation, and $74.4 \%$ reported not discussing AAC within a rotation.

Previous research also investigated resident's understanding and fulfillment of their role as an interdisciplinary team manager and care coordinator. Sneed et al., (2004) study indicated that physicians presented diagnoses and not much else. As discussed previously, this trend was also found within this study. 


\section{Implications of the Study}

This investigation has already proved informative, but the implications of these findings in light of current regulations, graduate medical educational expectations and resent research have yet to be discussed. More importantly, what are the possible implications of this study for the individuals in need of these services?

Each topic (e.g. regulations) has been previously covered within the literature review. Legislation, litigation and professional policies serve both as a catalyst for change and a shield to protect individuals receiving pediatric services. Specific organizations that provide such direction include the federal government, AAP, AMA, ACGME, Centers for Medicare and Medicaid Services (CMS) and the Joint Commission.

The implications of this study will focus on the findings regarding policies and regulations. Specifically, the areas of care coordination, AAC guidelines and new Centers for Medicare and Medicaid Services (CMS) and the Joint Commission regulations will be discussed.

\section{Care Coordination}

When comparing this study's data in light of the policies provided by the above organizations, the trend is both encouraging and discouraging. Most residents appear to fulfill the AAP policies of the medical home and care coordination in their perceived competence for identifying the need for speechlanguage therapy and $A A C$ as well as making a referral for either service. At the same time, when directly asked about their ability as a care coordinator for both 
communication disorders and AAC, most residents did not feel confident in their abilities.

It is unclear what questions these residents have about concerning care coordination. A variety of issues may be affecting residents' responses. During the informal qualitative portion of the study, the ability to find resources and gaps in resources were mentioned. Other possibilities were discussed within the literature including team dynamics, medical systems management, the care coordination process, and education (American Academy of Pediatrics Advisory Committee, 2002; American Academy of Pediatrics, 2002; Antonelli, \& Antonelli, 2004; Antonelli, Stille, \& Antonelli, 2008).

The lack of perceived confidence regarding care coordination within the pediatric population is concerning. Students come to a university expecting to receive training that will prepare them to competently fill their professional roles. Care coordination is an important pediatric role. This role is underscored by an American Academy of Pediatrics' policy as well as community experiences as a core component of residency curriculum (ACGME, 2007; Antonelli, \& Antonelli, 2004; Lypson, et al., 2004; Shipley, et al., 2005). If residents do not view themselves as competent in this area regarding communication disorders and $A A C$, then part of the education system requires change.

The results of this study supports previous research which indicated that barriers in the coordination process is in part due to a lack of medical student and resident training for care coordination skills (Antonelli, \& Antonelli, 2004; Antonelli, et al., 2008; McPherson et al., 2004). If residents are unsure of their 
ability to fulfill their role as a care coordinator the result may be incomplete, and episodic, expensive, fragmented care of children (Antonelli, \& Antonelli, 2004; Antonelli, et al., 2008; McPherson et al., 2004).

This lack of perceived competence at the pediatric resident level may have a compounding effect on the barriers within the care coordination system that already exist. Current barriers for care coordination include gaps in available resources, team dynamics, and medical systems management (Antonelli, \& Antonelli, 2004; Antonelli, Stille, \& Antonelli, 2008). Specifically, a lack of reimbursement, complex eligibility criteria, communication breakdown, language, economic and socio-cultural barriers, a lack team collaboration, and a lack of single point of entry into the medical system all stand in the way of care coordination and affect the provision of quality care within a pediatric practice (Antonelli, \& Antonelli, 2004; Antonelli, et al., 2008; McPherson et al., 2004). Within the world at large, knowledge of a policy does not equal compliance. Knowledge and compliance need to be instilled from the educational level to support the improved use of care coordination in new pediatricians, especially in light of the professional difficulties that lie ahead.

\section{AAC Guidelines}

Problems fulfilling the role as a care coordinator may cause concerns in the quality of services children receive. As pointed out within the AAP guidelines for AAC, the role of the pediatrician for AAC is an important responsibility (Desch, et al., 2008). Pediatricians should ensure access to appropriate augmentative and alternate communication services due to the complexity of the process for 
acquiring and using a communication device (Desch, et al., 2008). The AAP guidelines list the parts of the process which go beyond an initial referral. Desch (2008) was unable to provide information regarding the degree pediatricians understand their responsibility, or augmentative and alternate communication systems and services. Desch (2008) identified pediatric responsibilities as including writing letters of medical necessity, assisting with the implementation of the plan, finding and advocating for funding, device procurement, device training, and monitoring device use and therapy programs (Desch, et al., 2008).

The residents within the study were aware of the need for placing a referral, but did not appear as aware of their other responsibilities. The implications for this lack of knowledge are the same for a lack of care coordination, the possibility of incomplete, and episodic, expensive, fragmented care of children (Antonelli, \& Antonelli, 2004; Antonelli, et al., 2008; McPherson et al., 2004).

\section{Standards for Hospitals and Skilled Nursing Facilities}

Recent regulations have emerged from both the Centers for Medicare and Medicaid Services (CMS) and the Joint Commission to improve effective communication between professionals and patients (Joint Commission, 2010; Pressman, \& Blackstone, 2010). This regulation requires medical personnel to provided patients with an alternative means of communication, AAC, when they are not able to be understood or understand the communication of the medical professional. According to the CMS patients should be offered other communication means including but not limited to "writing, pointing or using cue 
cards" (Pressman, \& Blackstone, 2010, p. 7). The regulation suggests that skilled nursing facilities (SNF) staff has a "broad range of augmentative and alternative communication strategies and tools and other assistive technologies" at their disposal to assist with effective communication (Pressman, \& Blackstone, 2010, p. 7)

Within the discussion of the focus group, residents were unsure of these regulations. Their views appeared to focus on the immediate physical state of the client versus the communication needs with the client. As one resident stated,

"In an inpatient setting if the patient has a lisp or something else, if it is not a very acute issue this is not something speech helps us address in an inpatient setting. Because they are not being admitted because, because they have a lisp. You know, there is a bigger something going on. I think a lot of times that issue is dealt with as an outpatient."

However, it was unclear if the residents understood the new regulation or how it might impact their interaction with patients. The possible implications of a lack of knowledge could be communication breakdown between patients and professionals leading to "sentinel events, breaches of safety and reduced quality of care" (Pressman, \& Blackstone, 2010, p. 8). In light of the current state of consumer need, these implications could be negative.

\section{ACGME Competencies}

The constructs of this study were based on the six ACGME competencies. As can be seen in Appendix D, the ACGME competencies were directly used in the construction of each survey question. Each competency has specific requirements as well as guidelines for assessment (Joyce, 2006). The 
majority of responders indicated less perceived competence for the following ACGME competencies:

1. Medical Knowledge: V.A.5.b).(1).(f).(viii).(a), IV.A.5.b).(1).(c).(iv);

2. Patient Care: IV.A.5.a).(5).(f).(iii).(m) IV

3. Medical Education: IV.A.5.b).(1).(f).(viii).(c), V.A.5.b).(1).(f).(viii).(d).(i), IV.A.5.b).(1).(f).(viii).(d).(vi), IV.A.5.b).(1).(f).(viii).(b)

The competencies of Medical Knowledge and Medical Education are the focus of medical school and do not receive as much emphasis within residency training (ACGME, 2006; Joyce, 2006). The identification of these competencies as having a weakness is not surprising in light of residents' reports of diminished educational experiences for communication disorders and AAC. Further educational training with application this knowledge to patient care is needed.

The focus of patient care is regarding interaction with individual patients and the community. Residents are to provide compassionate, appropriate, and effective for the treatment of health problems and the promotion of health (ACGME, 2006). Patient care embodies care coordination. It again is not surprising that this ACGME competency is recognized due to resident's reporting weakness in their perceived competency for care coordination.

The ACGME does provide guidance on how to address areas of weakness. Systematic quality control is a required and important aspect of the Outcome project as it affects the educational system at the university level (ACGME, 2006; ACGME, 2007; Joyce, 2006). Medical schools must evaluate their educational program annually and keep documentation of annual meeting to 
review program goals and objectives and the effectiveness with which they are achieved (ACGME, 2006; ACGME, 2007; ACGME, 2010). When deficiencies are identified, an action plan is prepared (ACGME, 2006; ACGME, 2007). The program should use resident performance and outcome assessments to evaluate the educational effectiveness of the residency program (ACGME, 2006).

\section{Current Education Practices}

The pediatrics department within the University of Louisville has been in the forefront of training since the days of Abraham Flexner. Within the current training of pediatric residents, the results of this study indicate that individuals have some knowledge of their professional roles, such as making referrals and identifying a communication disorder or need for AAC. Residents' views towards their Professional Practice competency were unclear, but with an overall neutral response to all questions, there is room for improvement. Perceptions of Medical Education and Knowledge competency are changing over the course of pediatric levels. At the same time, residents reported a lack of educational training and diminished perceptions of competence for care coordination of these disabilities. These weaknesses may challenge the University's objective of current educational and continuing professional quality.

This study has provided insight regarding the strengths and weaknesses of resident education regarding communication disorders and $A A C$ within the University of Louisville. The identified weaknesses necessitate further assessment of how to improve resident training regarding communication disorders and AAC. Use of the systematic quality control as outlined by the 
Outcomes project is one option. Nevertheless, particular emphasis needs to be made on identification of the form and method of this training. Specifically, which rotations should provide this training, which educational format would be the most beneficial, and how much time is enough time for this topic.

\section{Limitations of the Study}

\section{Sample Size}

A number of limitations were identified within this study. The first issue is with the overall number of the participating population.

Return rate. The number of responders from within the population did not meet standard presented by Dillman as being representative (60\%). Only $43 \%$ of the population participated.

There were a number of confounding factors that may have caused issues such as a limited response. The first confounding factor was the timeline for the study's data collection. This occurred over the holiday season. A number of potential participants may have been on vacation and busy with holiday plans or events. This time frame may not have been the best for participants attention to the study's email.

The second confounding factor was the scheduled time for focus group. The time and place for the focus group was identified with the help of the pediatric resident education office. A scheduling error was made in that a prior conference was scheduled at same time as the focus group. Although residents received several emails regarding the focus group, the majority of residents went to the other scheduled conference. 
Another confounding factor was a lack of analysis of some data due to minimal responses (under 5 in group). Upon attempts to analyze the demographic information regarding the various forms of education experiences only one data set for AAC could be assessed. Within the body of pediatric residents, the number reporting direct educational experiences regarding AAC were: rotation $3(7 \%)$; ambulatory pediatric rotation, $1(2 \%)$; subspecialty rotation, $3(7 \%)$; morning report, $3(7 \%)$; core conference, $3(7 \%)$; and didactic $4(9 \%)$.

\section{Generalization}

Another limitation to this study was the ability for this data to be generalized to other institutions or populations. The information is applicable only to the current population at the University of Louisville. This study does not necessarily reflect on the regional or national population. Nor does this study reflect the educational practices of other universities.

These finding do not reflect on the professional abilities of practicing pediatricians either. Further investigation and replication of this study needs to occur for information to be applied to the pediatric resident community at large.

The overall population sample gathered is also problematic. With the small return rate, the degree to which these findings are applied to the population within the University of Louisville should proceed with caution.

\section{Future Research}

This investigation of pediatric residents has stirred up a number of questions for further inquiry. The foremost one being, will these results hold true for other graduate medical institutions? Replication of this study needs to occur 
on a wider scale to identify what, if any, changes in medical education may be required on an individual or national basis. This replication may also occur within the current institution to identify what impact additional educational opportunities may have on resident competency. Specific lines of inquiry may include identification of the form and method of resident training regarding communication disorders and AAC. Question may include which rotations should provide this training, which educational format would be the most beneficial, and how much time is enough time for this topic.

Other opportunities for investigation can be found in the community surrounding residency, namely pediatricians, other medical personnel, and consumers. The following research questions might be addressed:

1. If current pediatric residents demonstrate specific strengths and weaknesses, do practicing pediatricians respond in a similar fashion?

2. How competent is the medical support staff, such as nurses, in their identification of needs, especially within a hospital setting.

3. What are the consumers (user's and parent's) experience with pediatricians regarding $\mathrm{CD}$ and $\mathrm{AAC}$ ?

\section{Summary}

Without question, the ability to communicate is vital. Children with general communication impairments as well as complex communication needs rely on pediatricians to prescribe the services of speech language pathologists. In light of continuing and increasing needs, questions regarding pediatric resident's competence towards communication disorders and AAC were posed. 
To answer the pressing question of whether medical programs have addressed the need of residents for proper training in identifying communication disorders and the need for AAC, the answer is yes and no. Yes, there are some improvements within resident's perceived competence for referrals and knowledge base. Yet, it appears that changes are still needed regarding resident's educational opportunities and understanding of their role within the provision of services. Residents indicated care coordination as being a particular topic in need of attention.

Further assessment of how to improve resident training regarding communication disorders and AAC is needed. Use of the systematic quality control as outlined by the Outcomes Project is one option. Nevertheless, the most efficient form and method of training need to be identified. Follow-up of this current investigation by educational leaders and continued research within this field will support this effort. 


\section{REFERENCES}

ACGME (2000). ACGME at a glance [Data file]. Retrieved from http://www.acgme.org/acWebsite/newsRoom/newsRm acGlance.asp

ACGME (2000). ACGME fact sheet [Data file]. Retrieved from http://www.acgme.org/acWebsite/newsRoom/newsRm_factSheet.asp

ACGME (2000). Member organizations [Data file]. Retrieved from http://www.acgme.org/acWebsite/about/ab_memberOrg.asp

ACGME (2006). Introduction to competency-based education: Facilitator's guide [Data file]. Retrieved from http://www.acgme.org/acWebsite/about/ab_memberOrg.asp

ACGME (2006). ACGME outcome project: Introduction to [PPT]. Retrieved from www.acgme.org/outcome/project/OPintrorev1 7-05.ppt

ACGME (2007). Common program requirements: Pediatrics [Data file]. Retrieved from http://www.acgme.org/acWebsite/dutyHoursdh_dutyhoursCommon PR07012007.pdf

ACGME (2007). ACGME program requirements for graduate medical education in pediatrics [Data file]. Retrieved from http://www.acgme.org/acWebsite/downloads/RRC_progReq/320_pediatric S_07012007.pdf

ACGME (2010). Accreditation council for graduate medical education: Policies 
and procedures [Data file]. Retrieved from

http://www.acgme.org/acWebsite/about/ab ACGMEPoliciesProcedures.pd

f

Albanese, M., Mejicano,G., Mullan, P. Kokotailo, P. \& Gruppen, L. (2008).

Defining characteristics of educational competencies. Medical Education $42,248-255$.

Ambulatory Pediatric Association (2010). About us [Data file]. Retrieved from http://ambpeds.org/aboutUs/index.cfm

American Academy of Pediatrics (1999). The pediatrician's role in community pediatrics. Pediatrics, 103, $1304-1306$.

American Academy of Pediatrics (2002). The medical home: Policy statement. Pediatrics, 110, 184 - 186.

American Academy of Pediatrics Advisory Committee (2002). The medical home. Pediatrics, 110, $184-186$.

American Academy of Pediatrics (2003). Scope of practice issues in the delivery of pediatric health care. Pediatrics, 111(2), 426-435.

American Academy of Pediatrics (2005). Care coordination in the medical home: Integrating health and related systems of care for children with special health care needs. Pediatrics, 116(5), $1238-1244$.

American Academy of Pediatrics (2008). Pediatrics 101: A resource guide from the AAP (2008) [Data file]. Retrieved from http://www.aap.org/profed/Peds101book.pdf 
American Academy of Pediatrics Committee on Children with Disabilities (1998). Managed care and children with special health care needs: a subject review. Pediatrics, 102(3), 657-660.

American Academy of Pediatrics Committee on Community Health Services (1999). The pediatrician's role in community pediatrics. Pediatrics, 103(6), 1304-1306.

Association of American Medical Colleges (2010). Facts: Applicants, matriculants, enrollment, graduates, $M D / P h D$, and residency applicants data [Data file]. Retrieved from http://www.aamc.org/medicalschools.htm

American Council for Graduate Medical Education. 2007-2008 annual report accreditation council for graduate medical education: Number of accredited programs for the current academic year (2009 - 2010) [Data file]. Retrieved from http://www.acgme.org/adspublic/reports/accredited_programs.asp?accredi ted $=15$

American Medical Association (2008). How do you become a physician? [Data file]. Retrieved from http://www.amaassn.org/ama/pub/category/14365.html.

American Psychological Association. (2009). Publication Manual of the American Psychological Association (6 ed.). Washington, DC: Author. 
Antonelli, R., \& Antonelli, D. (2004). Providing a medical home: The cost of care coordination services in a community-based, general pediatric practice [Article], Pediatrics: American Academy of Pediatrics.

Antonelli, R., Stille, C., \& Antonelli, D. (2008). Care coordination for children and youth with special health care needs: a descriptive, multisite study of activities, personnel costs, and outcomes. Pediatrics, 122(1), 209-216.

American Speech-Language-Hearing Association (1993). Augmentative and alternative communication [Data file], Retrieved from http://www.asha.org/about/membership-certification/divs/div 12.htm American Speech-Language-Hearing Association (1993). About communication disorders [Data file]. Retrieved from http://www.asha.org/about/membership-certification/divs/div_12.htm American Speech-Language-Hearing Association (2004). Roles and responsibilities of speech-language pathologists with respect to alternative communication: Position statement [Data file], Retrieved on 20 November 2008 from:

http://www.asha.org/NR/rdonlyres/BA19B90C-1C17-4230-86A883B4E12E4365/0/v3PSaac.pdf

American Speech-Language-Hearing Association ASHA: The study of childhood language disorders in the $n$ Volume $26 ; 4$ 35-8

The Assistive Technology Training Online Project (ATTO). Using AAC devices [Data file]. Retrieved from $\leq$ http://atto.buffalo.edu/ $>$ 
Bailey, R., Parette, H., Jr., Stoner, J., Angell, M., \& Carroll, K. (2006). Family members' perceptions of augmentative and alternative communication device use. Language, Speech, and Hearing Services in Schools, 37(1), $50-60$.

Bailey, R, Stoner, J., Parette, H., \& Angell, M., (2007). AAC team perceptions: Augmentative and alternative communication device use. Education and Training in Developmental Disabilities, 41(2), 139-154. Beukelman D., \& Mirenda P. (2005). Augmentative and Alternative Communication: Supporting Children and Adults with Complex Communication Needs (3rd ed.). Baltimore: Paul H. Brookes Publishing Co.

Binger, C., \& Light, J. (2006). Demographics of preschoolers who require AAC. Language, Speech, and Hearing Services in Schools, 37(3), 200-208. Bingham, M., Spooner, F., \& Browder, D. (2007). Training paraeducators to promote the use of augmentative and alternative communication by students with significant disabilities. Education and Training in Developmental Disabilities, 42(3), 339-352.

Blackstone, S., Williams M., \& Joyce, M. (2002). Assistive technology: Future AAC technology needs: Consumer perspectives, Assistive Technology 14(1), $3-16$.

Brewer, E., Jr., McPherson, M., Magrab, P., \& Hutchins, V. (1989). Familycentered, community-based, coordinated care for children with special health care needs. Pediatrics, 83(6), 1055-1060. 
Cafiero, J. (2005). Meaningful exchanges for people with autism: An introduction to augmentative \& alternative communication. Bethesda, MD: Woodbine House.

Carmines, E, \& Zeller, R, (1979). Reliability and Validity Assessment:

Quantitative Applications in the Social Sciences. Thousand Oaks, CA:

Sage Publications, Inc.

Chomsky, N. (1968). Language and mind: Linguistic contributions to the study of mind: One of the six lectures is reproduced [Data file]. Retrieved from http://www.marxists.org/reference/subject/philosophy/works/us/chomsky.ht $\mathrm{m}$

Clarke, M., McConachie, H., Price, K., \& Wood, P. (2001). Speech and language therapy provision for children using augmentative and alternative communication systems. European Journal of Special Needs Education, 16(1), 41-54.

Collier, B., \& Blackstien-Adler, S. (1998). Building competencies in augmentative and alternative communication among professionals. AAC: Augmentative and Alternative Communication, 14(4), 250-260.

D'Aprix, R. (1982). The oldest (and best) way to communicate with employees. Harvard Business Review, 60(5), 30-32.

Desch, L., Gaebler-Spira, D., \& Disabilities, C. (2008). Prescribing assistivetechnology systems: Focus on children with impaired communication. Pediatrics, 121(6), 1271-1280.

Dillman, D., Smyth, J., Leah, M. (2009). Internet, Mail, 
and Mixed-Mode Surveys: The Tailored Design Method.

Hoboken, New Jersey: John Wiley \& Sons, Inc.

Division of Vital Statistics, National Center for Health Statistics, Centers for

Disease Control and Prevention (2009). Prevalence of autism spectrum

disorders-autism and developmental disabilities monitoring network,

United States, 2006 morbidity and mortality weekly report [Data file].

Retrieved from

http://www.cdc.gov/ncbddd/autism/data.htm|\#prevalence

Downing, J., \& Siegel-Causey, E., (1988). Enhancing the nonsymbolic communicative behavior of children with multiple impairments. Language, Speech and Hearing Services in Schools, 19(4), 338-348.

Duchan, J. (2002). Getting here: The first hundred years of speech pathology in America [Podcast]. Retrieved from www.acsu.buffalo.edu/ duchan/history.html <http://www.acsu.buffalo.edu/ duchan/history.html $>$.

Duchan J. (2009). The early years of language speech and hearing services. Language Speech and Hearing Services in Schools [Epub ahead of print]. Retrieved from http://lshss.asha.org/cgi/content/abstract/01611461_2009_08-0102v1

Drager, K., Light, J., Speltz, J., Fallon, K., \& Jeffries, L. (2003). The performance of typically developing 21/2-year-olds on dynamic display AAC technologies with different system layouts and language organizations. Journal of Speech, Language, and Hearing Research, 46(2), 298-312. 
Dynavox. $V$ and VMax (2008) [Data file]. Retrieved from $\leq \mathrm{http}: / / \mathrm{www} \cdot \mathrm{d}$ nnavoxtech.com/products/v/>

Education for All Handicapped Children's Act of 1975

Fallat, M, Glover, J. \& the Committee on Bioethics ( 2007). Professionalism in pediatrics: Technical report. Pediatrics, 120(4) e1123-e1133.

Fitch J., (1993). ASHA: Computer technology: History and overview. ASHA: A Journal of the American Speech-Language-Hearing Association. 35(9), 36-37.

Fromkin, V., Rodman, R., \& Hyams, N. (2003). An Introduction to Language: $7^{\text {th }}$ Edition. Boston, Massachusetts: Thomsom Heinle.

Galvin, J. \& Scherer, M., (1996). Evaluating, Selecting and Using Appropriate Assistive Technology. Gaithersburg Maryland: Aspen Publishers, Inc.

Golinker, L., (n.d.). Funding for Assistive Technology Devices and Services in the Individuals with Disabilities Education Act (IDEA) of 1997 [Data file]. Retrieved from http://www.ucp.org/ucp channeldoc.cfm/1/12/74/74-74/732

Goodman, D, \& the Committee on Pediatric Workforce (2005). Technical report: The pediatrician workforce: Current status and future prospects. Pediatrics, 116(1), 156-173.

Gupta, V., O'Connor, K., \& Quezada-Gomez, C. (2004). Care coordination services in pediatric practices, Pediatrics, 113(5), 1517-1521. 
Henderson, C. (2000). 1990s Profile of Students with Disabilities in Higher

Education [Data file]. Retrieved from

http://ehrweb.aaas.org/entrypoint/rr/appendix2.html

Higginbotham, D., Shane, H., Russell, S., \& Caves, K. (2007). Access to AAC:

Present, past, and future AAC: Augmentative and Alternative

Communication, 23(3), 243-257.

Hill, K. (2004). Augmentative and alternative communication and language:

Evidence-based practice and language activity monitoring. Topics in Language Disorders, 24(1), 18-30.

Hill, K., \& Romich, B. (2001). A Language activity monitor for supporting AAC evidence-based clinical practice. Assistive Technology, 13(1), $12-22$.

Hill, K., Romich, B., Seagull, A., Ahmad, N., Peebles, B., \& Strecker, J. (2003). AAC internet-based self-study shell: Proceedings of the 2003 RESNA Conference. Arlington, VA.: RESNA Press.

Houser, M., Horan, S., \& Furler, L. (2008). Dating in the fast lane: How communication predicts speed-dating success. Journal of Social and Personal Relationships, 25(5), 749-768.

International Society for Augmentative and Alternative Communication. (2006). Who we are [Data file]. Retrieved on 5 June 2009 from: http://www.isaac-online.org/en/about/who.html Jenkins, R. (2003). Resident training and education in the United States. [peer reviewed]. Pediatrics, 112, $752-754$. 
Johnson, J., Inglebret,, Jones, C., \& Ray, J. (2006). Perspectives of speech language pathologists regarding success versus abandonment of AAC. AAC: Augmentative and Alternative Communication, 22(2), 85-99.

Jones, S. (2004). Review of augmentative and alternative communication: Management of severe communication disorders in children and adults. Journal of Applied Research in Intellectual Disabilities, 17(2), 133-134.

Joyce, B. (2006). Introduction to competency-based education facilitator's guide: ACGME [Data file]. Retrieved from http://www.ama-assn.org/ama/pub/education-careers/becomingphysician.shtml

Joyce, B. (2005). ACGME implementation toolbox [Data file]. Retrieved from www.acgme.org/outcome/implement/impHome.asp

Justice, L. (2006). Communication Sciences and Disorders: An Introduction. Upper Saddle River: Pearson Education, Inc.

KATS Network, (2008). About the KATS network [Data file]. Retrieved from http://www.katsnet.org/about/

Kaczorowski, J. (2008). Pediatrics in the community: community pediatrics training initiative (CPTI). Pediatric Review, 29(1), 31-32.

Koenning, G., Benjamin, J., Todaro, A., Warren, R., \& Burns, M. (1995). Bridging the "med-ed gap" for students with special health care needs: A model school liaison program. Journal of School Health, 65(6), 207-212. 
Larcher, J. (2004). Review of augmentative and alternative communication developmental issues. Child Language Teaching \& Therapy, 20(3), 331333.

Lebel, T. Olshtain, E., \& Weiss, P. (2005). Teaching teachers about augmentative and alternative communication: Opportunities and challenges of a web-based course. AAC: Augmentative and Alternative Communication, 21(4), 264-277.

Liaison Committee on Medical Education (2010). Directory of accredited medical education programs [Data file]. Retrieved from http://www.lcme.org/directry.htm

Light J., Beukelman D., \& Reichle J (2003). Communicative Competence for Individuals Who Use AAC: From Research to Effective Practice. Baltimore: Paul H. Brookes Publishing Co.

Light, J., Drager, K., McCarthy, J., Mellott, S., Millar, D., Parrish, C., et al. (2004). Performance of typically developing four- and five-year-old children with AAC systems using different language organization techniques. $A A C$ : Augmentative and Alternative Communication, 20(2), 63-88.

Lloyd, L., Fuller, D., \& Arvidson, H. (1997). Augmentative and Alternative Communication: A Handbook of Principles and Practices. Needham Heights, MA: Allyn and Bacon.

Lypson, M., Frohna, J., Gruppen, L., \& Woolliscroft, J. (2004). Assessing residents' competencies at baseline: identifying the gaps. Academic Medicine, 79(6), 564-570. 
McPherson M., Arango P., Fox H., Lauver C., McManus M., Newacheck P., Perrin J., Shonkoff J., \& Strickland B. (1998). A new definition of children with special health care needs. Pediatrics, 102(1):137-140.

McPherson, M., Weissman, G., Strickland, B., van Dyck, P., Blumberg, S., \& Newacheck, P. (2004). Implementing community-based systems of services for children and youths with special health care needs: How well are we doing? Pediatrics, $113(5$ Supplementary), 1538-1544.

Marshall, J. \& Heffes, E, (2006). Employee communication linked to performance. Financial Executive, 22(1), 11.

Martin J., Kung H., Mathews T., Hoyert D., Strobino D., Guyer B., \& Sutton S., (2008). Annual summary of vital statistics: 2006. Pediatrics, 121(4), 788-801.

Melnyk, B., Fineout-Overholt, E., Hockenberrry, M., Huth, M., Jamerson, P., Latta, L., et al. (2007). Improving healthcare and outcomes for high-risk children and teens: formation of the National Consortium for Pediatric and Adolescent Evidence-Based Practice. Pediatric Nurse, 33(6), 525-529.

Microsoft Corporation (2009). Encarta World English Dictionary [North American Edition]. Bloomsbury Publishing Plc.

Millar, D., Light, J., \& Schlosser, R. (2006). The impact of augmentative and alternative communication intervention on the speech production of individuals with developmental disabilities: A research review. Journal of Speech, Language, and Hearing Research, 49,248-264. 
Mirenda, P. (1997). Supporting individuals with challenging behavior through functional communication training and AAC: Research review. AAC: Augmentative and Alternative Communication, 13(4), 207-225.

Mirenda, P. (2001). Autism, augmentative communication, and assistive technology: What do we really know? Focus on Autism and Other Developmental Disabilities, 16, 141-151.

Mirenda, P. (2003). Toward a functional augmentative and alternative communication for students with autism: Manual signs, graphic symbols, and voice output communication aids. Language, Speech, and Hearing Services in Schools, 34(3), 203-216.

Mirenda, P. \& lacono, T. (2009). Autism Spectrum Disorders and AAC. Baltimore, MD: Paul H. Brookes Publishing Co., Inc.

Moore, B., \& Tonniges, T. (2004). The "every child deserves a medical home" training program: More than a traditional continuing medical education course. Pediatrics, 113(5), 1479-1484.

Mosby's Medical Dictionary, 8th edition. (2009), Elsevier.

Mulvihill, B. A., Altarac, M., Swaminathan, S., Kirby, R. S., Kulczycki, A., \& Ellis, D. E. (2007). Does access to a medical home differ according to child and family characteristics, including special-health-care-needs status, among children in Alabama? Pediatrics, 119 Supplemental 1, S107-113.

Meyer. P., (n.d.). BrainyQuote.com [Data file]. Retrieved from http://www.brainyquote.com/quotes/quotes/p/paulmeyer190945.html National Council on Disability (2005). Lessons for all of us: Protecting the right 
to education for persons with disabilities [Data file]. Retrieved from http://www.ncd.gov/newsroom/publications/2005/lessons.htm

National Information Center for Children and Youth with Disabilities (NICHCY) (2005). The education of children and youth with disabilities: What do the laws say? [Data file]. Retrieved fromhttp://www.nichcy.org/pubs/newsdig/nd26.htm

National Information Center for Children and Youth with Disabilities (2009). Speech and language impairments [Data file]. Retrieved from http://www.nichcy.org/Disabilities/Specific/Pages/SpeechLanguagelmpair ments.aspx

National Institute on Deafness and Other Communication Disorders National Institutes of Health (2002). What Is Voice? What Is Speech? What Is Language? [Data file]. Retrieved from http://www.nidcd.nih.gov/health/voice/whatis vsl.htm\#4

National Research Council. (2001). Educating Children with Autism. Washington, DC: National Academy Press.

No author (n.d.). American with disabilities act: 15 years [Data file]. Ability Magazine, Retrieved from http://abilitymagazine.com/american disabilities.html

Resident Matching Program and Association of American Medical Colleges (2009). Charting outcomes in the match: Characteristics of applicants who matched to their preferred specialty in the 2009 main 
residency match; 3rd edition [Data file]. Retrieved from http://www.nrmp.org/data/programresults2006-2010.pdf

Reily L., Panhan H., \& Tupinambá, A., (2009). Early evidence of low-tech communication in an Ott painting. Augmentative and Alternative Communication, 25(4), 217-224.

Rolnick, S., Flores, S., O'Fallon, A., \& Vanderburg, N. (2000). The implementation of clinical guidelines in a managed care setting: implications for children with special health care needs. Managed Care Quarterly, 8(2), 29-38.

Sadof, M., \& Nazarian, B. (2007). Caring for children who have special healthcare needs: a practical guide for the primary care practitioner. Pediatrics Review, 28(7), e36-42.

Schlosser, R. (2003). Roles of speech output in augmentative and alternative communication: Narrative review. Augmentative and Alternative Communication, 19, 5-28.

Schlosser, R. (2003). The Efficacy of Augmentative and Alternative Communication Intervention: Toward Evidence-Based Practice. San Diego, CA: Academic Press.

Schlosser, R., \& Raghavendra, P. (2004). Evidence-based practice in augmentative and alternative communication. AAC: Augmentative and Alternative Communication, 20(1), 1-21.

Schlosser, R., \& Wendt, O. (2008). Effects of augmentative and alternative 
communication intervention on speech production in children with autism:

A systematic review. American Journal of Speech-Language Pathology, $17,212-230$.

Sigafoos, J., Schlosser, R., \& Sutherland, D., (2011). Augmentative and alternative communication. International Encyclopedia of Rehabilitation. Retrieved from http://cirrie.buffalo.edu/encyclopedia/en/article/50/

Section 504 of the Rehabilitation Act of 1972, as amended, 29 U.S.C. $\S 794(a)$ Section 508 (2008). 508 Law [Data file]. Retrieved from http://www.section508.gov/index.cfm?FuseAction=Content\&ID=3

Shipley, L. J., Stelzner, S. M., Zenni, E. A., Hargunani, D., O'Keefe, J., Miller, C., et al. (2005). Teaching community pediatrics to pediatric residents:

Strategic approaches and successful models for education in community health and child advocacy. Pediatrics, 115(4), 1150-1157.

Sia, C., Tonniges, T. F., Osterhus, E., \& Taba, S. (2004). History of the medical home concept. Pediatrics, 113, 1473-1478.

Simpson, R., \& Zionts, P. (2000). Autism: Information and Resources for Professionals and Parents. Austin, TX: Pro Ed.

Sneed, R., May, W., \& Stencel, C. (2000). Training of pediatricians in care of physical disabilities in children with special health needs: Results of a twostate survey of practicing pediatricians and national resident training programs. Pediatrics, 105(3 Pt 1), 554-561.

Sneed, R., May, W., \& Stencel, C. (2001). Physicians' reliance on specialists, therapists, and vendors when prescribing therapies and durable medical 
equipment for children with special health care needs. Pediatrics, 107(6), 1283-1290.

Sneed, R., May, W., \& Stencel, C. (2004). Policy versus practice: Comparison of prescribing therapy and durable medical equipment in medical and educational settings. Pediatrics, 114(5), 612-625.

Sneed, R., May, W., Stencel, C., \& Paul, S. (2002). Pediatric physiatry in 2000: A survey of practitioners and training programs. Archives of Physical and Medical Rehabilitation, 83(3), 416-422.

SurveyMonkey.com, LLC, (2010). Palo Alto, California, USA. Retrieved from http://www.surveymonkey.com/

Starfield, B., \& Leiyu, S. (Writer) (2004). The medical home, access to care, and insurance: A review of evidence, Pediatrics: American Academy of Pediatrics.

Stille, C. J., \& Antonelli, R. (2004). Coordination of care for children with special health care needs. Current Opinion in Pediatrics, 16(6), 700-705.

The American Heritage Medical Dictionary (2007), Houghton Mifflin Company.

The ARC (2011). History of the ARC: The power of parents [Data file]. Retrieved from http://www.thearc.org/page.aspx?pid=2338

University of Louisville (2008). The history of the school of medicine [Data file]. Retrieved from http://louisville.edu/medschool/about/history/ University of Louisville (2011). Educational experiences [Data file]. 
Retrieved from

http://louisville.edu/medschool/pediatrics/residency/program-

overview/educational-experiences.html

University of Louisville (2011). Rotations and electives [Data file].

Retrieved from

http://louisville.edu/medschool/pediatrics/residency/program-

overview/rotations-and-electives.html

U.S.Census Bureau (2007). Facts for features \& special editions May 29, 2007 [Data file]. Retrieved from

http://www.census.gov/newsroom/releases/archives/facts for features sp ecial editions/index.html

U. S. Department of Education (2006). U.S. department of education annual report to congress on the assistive technology act of 1998 for fiscal years 2004 and 2005 [Data file]. Retrieved from www.ed.gov

U.S. Department of Education (2008). Twenty-sixth annual report to congress on the implementation of the individuals with disabilities education act (2008) [Data file]. Retrieved from shttp://www.ed.gov/about/reports/annual/osep/2004/index.html>

U.S. Department of Education (2009). Protecting students with disabilities: Frequently asked questions about section 504 and the education of children with disabilities [Data file]. Retrieved from http://www2.ed.gov/print/about/offices/list/ocr/504faq.html

U.S. Department of Education (n.d.) 2007 americans with disabilities act [Data 
file]. Retrieved from http://idea.ed.gov/

U.S. Department of Education National Institute on Disability and Rehabilitation Research (2005). Assistive technology and information technology use and need by persons with disabilities in the United States, 2001 [Data file]. Retrieved from http://www.edpubs.org

U.S. Department of Education Office of Civil Rights (1998). Auxiliary aids and services for post-secondary students with disabilities: Higher education's obligation under Section 504 and Title II of ADA [Data file]. Retrieved from http://www.ed.gov/offices/OCR/auxaids.html

U.S. Department of Education Office of Civil Rights (1995). The civil rights of students with hidden disabilities under section 504 of the rehabilitation act of 1973 [Data file]. Retrieved from http://www.ed.gov/offices/OCR/hq5269.html

U. S. Department of Education Office of Civil Rights (1999). Impact of the civil rights laws [Data file]. Retrieved from http://www.ed.gov/offices/OCR/impact.html

U.S. Department of Education Office for Civil Rights (1999). Impact of the civil rights: Removing disability barriers to educational opportunity [Data file]. Retrieved from http://www2.ed.gov/print/about/offices/list/ocr/docs/impact.html

U.S. Department of Education, Office of Special Education Programs (1995). Postsecondary students with disabilities: Where are they enrolled? American Council on Education Research Brief Series, 6(6). 
U.S. Department of Education, Office of Special Education Programs.

Nineteenth annual report to congress on the implementation of the individuals with disabilities education act, 1997, page A-1; IDEA's definition of disabilities [Data file]. Retrieved from http://www.hoagiesgifted.org/eric/e560.html

U.S. Department of Education Office of Special Education and Rehabilitative Services Rehabilitation Services Administration (2001). Information memorandum: RSA -IM - 02-01 [Data file]. Retrieved from http://www.lcme.org/directry.htm

United States Department of Education Office of Special Education and Rehabilitative Services (2005). History twenty-five years of progress in educating children with disabilities through IDEA [Data file]. Retrieved from www.ed.gov/offices/osers/osep

United States Department of Veterans Affairs (n.d.). VA History in Brief [Data file]. Department of Veterans Affairs Office of Public Affairs (80); Washington, DC Retrieved from

United States Medical Licensing Examination (2010). About USMLE [Data file]. Retrieved from http://www.usmle.org/General_Information/general_information_about.htm 1

Weiner, P. S. (1984). Overview: Accreditation and the LCME [Data file]. Retrieved from http://www.lcme.org/directry.htm

West, R. (1984). Association: The association in historical perspective. 
ASHA, 26, (supplemental) $7-9$.

Wetherby, A.M. \& Prizant, B. (2000). Autism Spectrum Disorders: A Transactional Developmental Perspective. Baltimore, MD: Paul H. Brookes Publishing Co., Inc.

Wilkinson, K., \& Henning, S. (2007). The state of research and practice in augmentative and alternative communication for children with developmental disabilities. Mental Retardation and Developmental Disabilities Research Reviews, 13,58-69.

Ziring, P. R., Brazdziunas, D., Cooley, W. C., Kastner, T. A., Kummer, M. E., Gonzalez de Pijem, L., et al. (1999). American academy of pediatrics. committee on children with disabilities. Care coordination: integrating health and related systems of care for children with special health care needs. Pediatrics, 104(4 Pt 1), 978-981. 
Appendix A

Cronbach's Alpha 


\section{Cronbach's Alpha}

Cronbach's alpha was conducted to further determine if items in each construct measured the same concept (Nunnally \& Bernstein, 1994).

Coefficients were found to range from .78 to .95 . Medical Education produced a Cronbach's alpha coefficient of .864 , Medical Knowledge produced a Cronbach's alpha coefficient of .716, and Professional Practice produced a Cronbach's alpha coefficient of 837 . All three constructs exceeded a minimum value of .70 as suggested by Nunnally nd Bernstein (1994). A complete listing of the Cronbach's alpha scores for all scales can be found in Tables14, 15 and 16.

\section{Medical Education.}

The first construct was education. Specifically within this study, education referred to graduate medical education. This construct corresponded with the following ACGME competencies: Medical Knowledge, Patient Care, and Medical Education. The construct was calculated from students' answers to questions one through five from the PR:CDAAC survey. The Likert scale answer reflected the students' perceptions about their pediatric resident educational training experiences for communication disorders and AAC in compliance with ACGME competencies. Table 1 presents results of the reliability analysis for this scale.

The Composite mean of 2.71 indicates that the responses for these items were tending toward centralization. Cronbach's alpha for the scale is .864 , an acceptable value, especially given the special nature of this population. 
Table 20

Psychometric Analysis for Medical Education ( $N=43)$

\begin{tabular}{llccccc}
\hline Item & $M$ & $S D$ & Min & Max & $\mathrm{R}$ & $\alpha-\mathrm{d}$ \\
\hline Q1R & 2.58 & 1.33 & 1 & 5 & 4 & .880 \\
Q2R & 2.95 & 1.19 & 1 & 5 & 4 & .837 \\
Q3R & 2.65 & 1.09 & 1 & 5 & 4 & .810 \\
Q4R & 2.77 & 1.02 & 1 & 5 & 4 & .828 \\
Q5R & 2.58 & 1.03 & 1 & 5 & 4 & .825 \\
Composite & 2.71 & .591 & 2.58 & 2.95 & .37 & .873
\end{tabular}

Note. Min $=$ Minimum; Max $=$ Maximum $; \mathrm{R}=$ Range $; \alpha-\mathrm{d}=$ alpha with item deleted.

Note. value for composite for $\mathrm{a}-\mathrm{d}$ is Cronbach's coefficient alpha for overall scale.

\section{Medical Knowledge}

The next construct investigated was knowledge. Knowledge, as defined by the Encarta@ World English Dictionary [North American Edition] (2009), is a general awareness or possession of information, facts, ideas, truths, or principles. This construct investigated resident knowledge of communication disorders and AAC in compliance with the ACGME competencies of Medical Knowledge, Patient Care and Medical Education.

The construct was calculated from students' answers to questions six through eleven from the PR:CDAAC survey. The Likert scale answer reflected the students' perceptions about their pediatric resident knowledge for communication disorders and AAC in compliance with ACGME competencies. Table 2 presents results of the reliability analysis for this scale. The Composite mean of 3.34 indicates that the responses for these items were tending toward centralization. Cronbach's alpha for the scale is .72, an acceptable value, especially given the special nature of this population. 
Table 21

Psychometric Analysis for Medical Knowledge ( $N=43)$

\begin{tabular}{llllcll}
\hline Item & $M$ & $S D$ & Min & Max & $\mathrm{R}$ & $\alpha-\mathrm{d}$ \\
\hline Q6R & 3.79 & .67 & 1 & 5 & 4 & .880 \\
Q7R & 3.88 & .70 & 1 & 5 & 4 & .837 \\
Q8R & 2.70 & 1.04 & 1 & 5 & 4 & .810 \\
Q9R & 2.41 & .96 & 1 & 5 & 4 & .828 \\
Q10R & 3.88 & .76 & 1 & 5 & 4 & .825 \\
Q11R & 3.35 & 1.09 & 1 & 5 & 4 & .825 \\
Composite & 3.34 & .591 & 2.42 & 3.88 & 1.47 & .716 \\
\hline
\end{tabular}

Note. Min = Minimum; Max $=$ Maximum; $R=$ Range $; \alpha-d=$ alpha with item deleted.

Note. Value for composite for $\alpha-d$ is Cronbach's coefficient alpha for overall scale.

\section{Professional Practice}

The final construct addressed was professional practice. The American Board of Pediatrics (ABP) developed guidelines for the teaching and evaluation of professionalism, or professional practice, as part of the core curriculum for residency training in pediatrics (Fallat, \& Glover, 2007, pg. e1124). These guidelines overlap and accentuate five of the six ACGME competencies including the following: Patient Care, Professionalism, Interpersonal and Communication Skills, Practice-Based Learning and Improvement and System-Based Practice. Professional practice is distinctive from medical knowledge. The following eight components of professional practice are endorsed by the ABP: honesty and integrity, reliability and responsibility, respect for others, compassion/ empathy, self-improvement, self-awareness/ knowledge of limits, communication and collaboration and altruism and advocacy (Fallat, et al., 2007). 
The construct was calculated from students' answers to questions twelve through thirty-one from the PR:CDAAC survey. The Likert scale answer reflected the students' perceptions about their pediatric resident educational training experiences for communication disorders and AAC in compliance with ACGME competencies. Table 13 presents results of the reliability analysis for this scale. The Composite mean of 3.07 indicates that the responses for these items were tending toward centralization. Cronbach's alpha for the scale is .94 , an acceptable value, especially given the special nature of this population.

Table 22

Psychometric Analysis for Professional Practice $(N=40)$

\begin{tabular}{lllllll}
\hline Item & $M$ & $S D$ & Min & Max & $\mathrm{R}$ & $\alpha-\mathrm{d}$ \\
\hline Q12R & 3.88 & .72 & 1 & 5 & 4 & .94 \\
Q13R & 3.63 & .87 & 1 & 5 & 4 & .94 \\
Q14R & 3.23 & .97 & 1 & 5 & 4 & .93 \\
Q15R & 3.03 & .97 & 1 & 5 & 4 & .93 \\
Q16R & 3.03 & .97 & 1 & 5 & 4 & .93 \\
Q17R & 2.78 & .92 & 1 & 5 & 4 & .93 \\
Q18R & 2.98 & .80 & 1 & 5 & 4 & .93 \\
Q19R & 2.85 & .86 & 1 & 5 & 4 & .93 \\
Q20R & 3.15 & .83 & 1 & 5 & 4 & .93 \\
Q21R & 3.08 & .80 & 1 & 5 & 4 & .93 \\
Q22R & 3.25 & .81 & 1 & 5 & 4 & .93 \\
Q23R & 2.83 & .96 & 1 & 5 & 4 & .93 \\
Q24R & 3.60 & .67 & 1 & 5 & 4 & .94 \\
Q25R & 3.25 & .93 & 1 & 5 & 4 & .94 \\
Q26R & 3.33 & 1.16 & 1 & 5 & 4 & .94 \\
Q27R & 3.00 & 1.20 & 1 & 5 & 4 & .94 \\
Q28R & 3.00 & .93 & 1 & 5 & 4 & .93 \\
Q29R & 2.93 & .89 & 1 & 5 & 4 & .93 \\
Q30R & 2.33 & .80 & 1 & 5 & 4 & .94 \\
Q31R & 2.33 & .80 & 1 & 5 & 4 & .94 \\
Composite & 3.07 & .591 & 2.33 & 3.88 & 1.55 & .94 \\
& & & & & & \\
\hline
\end{tabular}

Note. Min $=$ Minimum; Max $=$ Maximum; $\mathrm{R}=$ Range; $\alpha-\mathrm{d}=$ alpha with item deleted. 
Note. Value for composite for $\alpha$ - $d$ is Cronbach's coefficient alpha for overall scale. 
Appendix B

Dependent Variable Histograms 


\section{Dependent Variable Histograms}

\section{Figure 1}

Histogram of Frequencies for Medical Education Competence for Pediatric Levels One, Two and Three

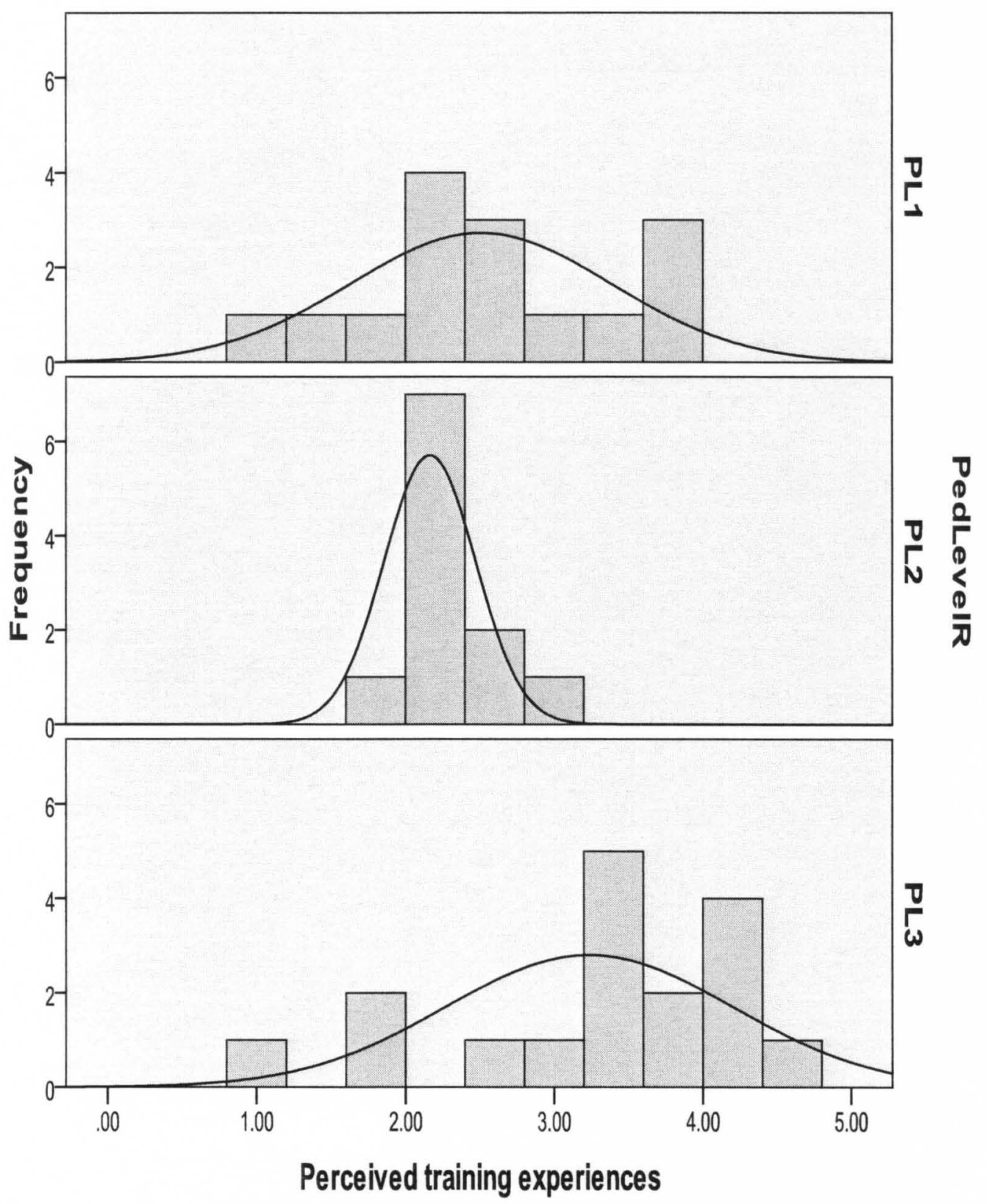


Figure 2

Histogram of Frequencies for Medical Knowledge Competence for Pediatric Levels One, Two and Three

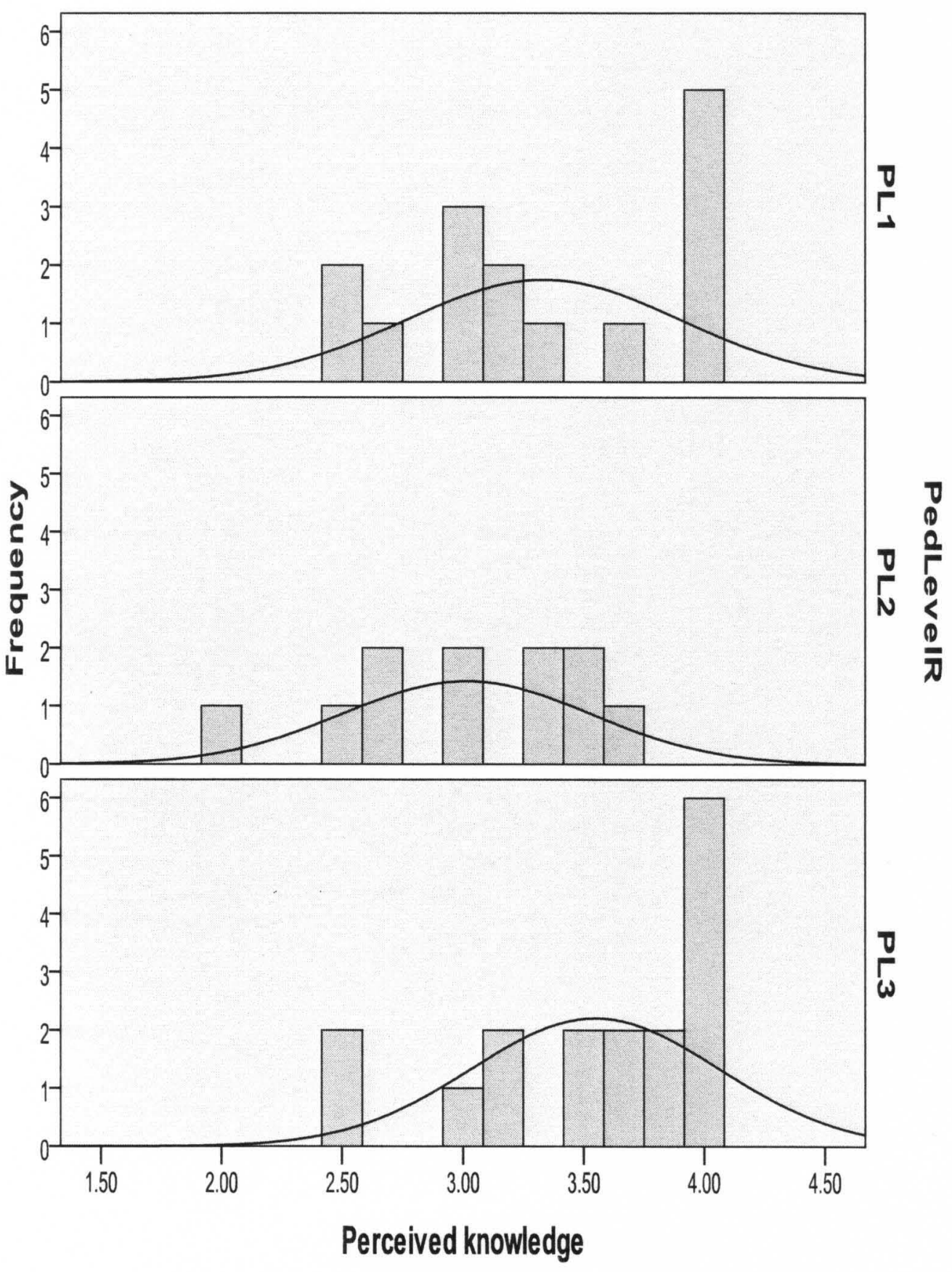


Figure 3

Histogram of Frequencies for Professional Practice Competence for Pediatric Levels One, Two and Three

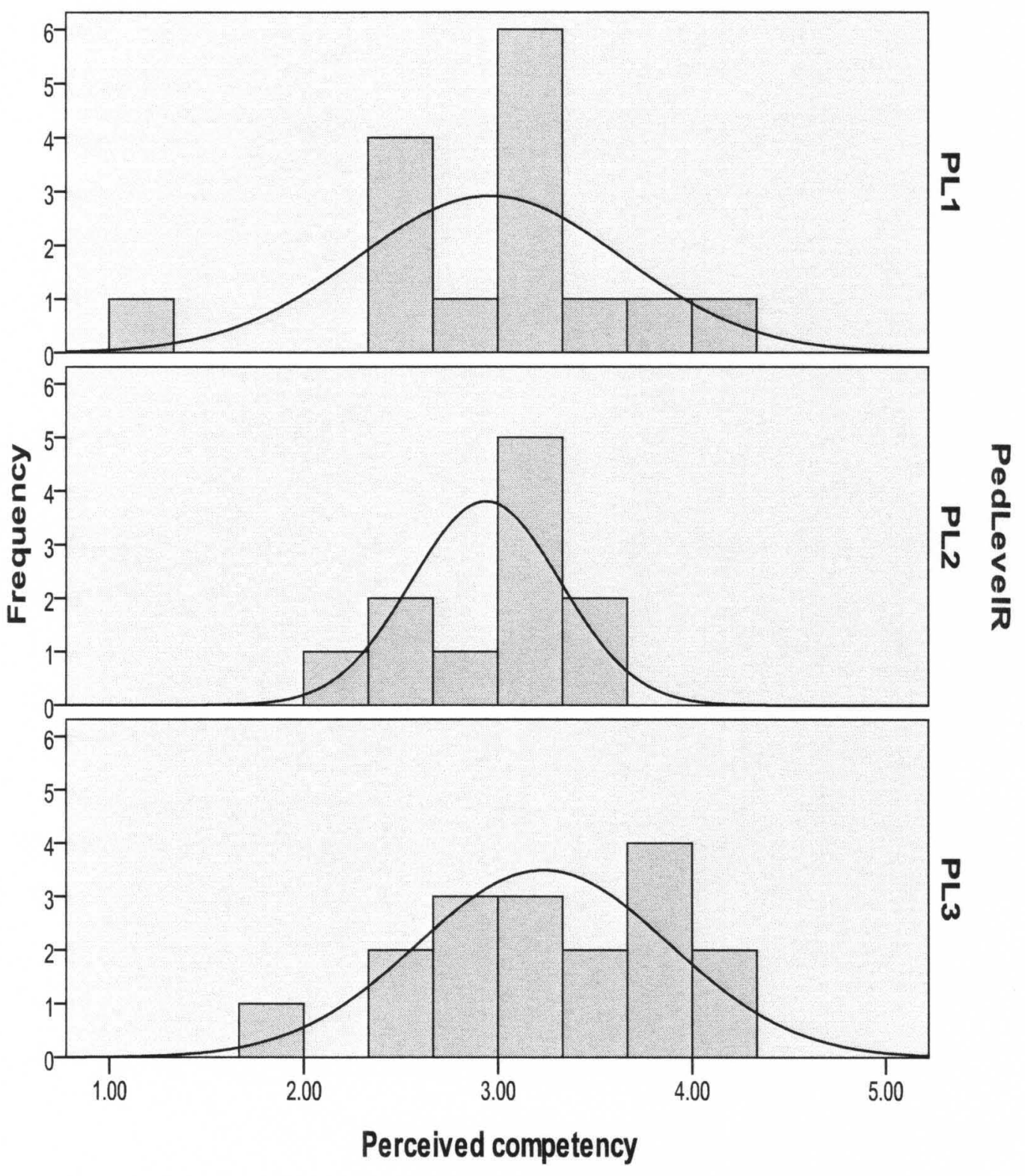


Appendix C

Demographic Variables 


\section{Demographic Variables}

The fourth research question investigated the effects of demographic variables on residents' perceived competency across the three constructs. The five demographic variables include the following: gender, rotation completion, pediatric specialization educational methods, and educational time.

Independent $t$ tests and correlation coefficients were used to analyze this

data. The dependent variables were residents' perceived competency (the average scores on the three constructs obtained from the questionnaire) and the independent variables were demographic variables within the survey (gender, rotation completion, pediatric specialization educational methods, and educational time).

The software package SPSS version 15 Windows (SPSS, 2003), provided the computational analysis. The same software package was used for data entry by the investigator. All statistical analyses used .05 as the level of significance. However, to obviate the inflation of Type I error rate due to repeated statistical tests, the Bonferroni correction was used. It will be implemented in the following manner. The three dependent variables were defined as a set. Within this set, the overall error rate was kept at .017 by dividing the number of tests into .05 and using the resulting value as the alpha level to be used for each comparison. Thus, the level of significance for all $t$ tests was $p=.017$. 


\section{Demographic Variable Gender}

Males and females were compared on the average levels of self-reported competence in the three constructs regarding communication disorders and augmentative and assistive technology.

Assumptions. The ability of the data to meet methodological assumptions was assessed. The assumptions for an independent $t$ test included independence, normality, and equality of variances. In this study, all observations were independent as each participant completed a single survey. Due to the robustness of a $t$ - test, with two assumptions met, a histogram for the data points was not performed.

Levene's test gauged the homogeneity of variance among the variables. For all three constructs, the Levene's test was not significant at the .05 level: Medical Education (Levene's, $F=3.15, p=.083, p>.05$ ), Medical Knowledge (Levene's, $F=1.81, p=.186, p>.05$ ), Professional Practice (Levene's, $F=.36$, $p=.55, p>.05)$. There was not a violation of homogeneity, and the assumption for homogeneity of variance was met.

Independent $t$ tests. The initial $t-$ test investigated how males and females were compared on the average levels of self-reported competence in the three constructs regarding communication disorders and augmentative and assistive technology. Table 23 presents information regarding the frequency, mean, standard deviation and standard error of the mean for gender across the various constructs. 
Table 23

Descriptive Statistics for Gender $(N=43)$

\begin{tabular}{llllll} 
DV & Gender & $\#$ & Mn & SD & SEM \\
\hline E & M & 17 & 2.94 & 1.06 & .26 \\
& $\mathrm{~F}$ & 26 & 2.55 & .80 & .16 \\
$\mathrm{~K}$ & $\mathrm{M}$ & 17 & 3.31 & .64 & .16 \\
& $\mathrm{~F}$ & 26 & 3.35 & .52 & .10 \\
$\mathrm{P}$ & $\mathrm{M}$ & 17 & 3.23 & .61 & .15 \\
& $\mathrm{~F}$ & 26 & 2.96 & .60 & .12
\end{tabular}

Note. $\mathrm{DV}=$ Dependent Variable; $\mathrm{E}=$ Medical Education; $\mathrm{K}=$ Medical Knowledge; $\mathrm{P}=$ Professional Practice

Note. $\#=$ frequency; $\mathrm{Mn}=$ Mean; $\mathrm{SD}=$ Standard Deviation; $\mathrm{SEM}=$ Standard Error Mean

Note. $\mathrm{M}=$ Male; $\mathrm{F}=$ Female

Upon analysis of the $t$ test, a significant difference was not discovered between gender for any of the constructs: Medical Education, $t(41)=1.37, p=$ $.18, p>.017 ;$ Medical Knowledge, $t(41)=-.22, p=.83, p>.017 ;$ and Professional Practice, $t(41)=1.45, p=.15, p>.017$. There was not a significant relationship between gender and self-reported competence across the three constructs.

\section{Demographic Variable Pediatric Specialization}

Various specializations were coded as either Yes or No and the two groups were compared on the average levels of self-reported competence in the three constructs regarding communication disorders and augmentative and assistive technology.

Assumptions. The ability of the data to meet methodological assumption was assessed. The assumptions for an independent $t$ test included 
independence, normality, and equality of variances. In this study, all observations were independent as each participant completed a single survey. Due to the robustness of a $t$ - test, with two assumptions met, a histogram for the data points was not performed.

Levene's test gauged the homogeneity of variance among the variables.

For all three constructs, the Levene's test was not significant at the .05 level: Medical Education (Levene's, $F=.09, p=.77, p>.05$ ), Medical Knowledge (Levene's, $F=.05, p=.83, p>.05$ ), Professional Practice (Levene's, $F=.00, p=$ $.99, p>.05)$. There was not a violation of homogeneity, and the assumption for homogeneity of variance was met.

Independent $t$ tests. The $t$ - test investigated how pediatric residents identifying a specialization versus those that did not compared on the average levels of self-reported competence in the three constructs regarding communication disorders and augmentative and assistive technology. Within the body of pediatric residents, the population pursuing a specialization totaled 24 $(55.8 \%)$. Those who indicated 'no' totaled $19(44.2 \%)$. For information regarding those specific specializations, see Tables 3 and 4 . Table 24 presents information regarding the frequency, mean, standard deviation and standard error of the mean for gender across the various constructs. 
Table 24

Descriptive Statistics for Specialization $(N=43)$

\begin{tabular}{lccccc} 
DV & Specialization & $\#$ & Mn & SD & SEM \\
\hline E & Y & 19 & 2.87 & .90 & .21 \\
& $\mathrm{~N}$ & 24 & 2.58 & .93 & .19 \\
$\mathrm{~K}$ & $\mathrm{Y}$ & 19 & 3.48 & .57 & .13 \\
& $\mathrm{~N}$ & 24 & 3.22 & .54 & .11 \\
$\mathrm{P}$ & $\mathrm{Y}$ & 19 & 3.22 & .57 & .13 \\
& $\mathrm{~N}$ & 24 & 2.94 & .62 & .13
\end{tabular}

Note. $\mathrm{DV}=$ Dependent Variable; $\mathrm{E}=$ Medical Education; $\mathrm{K}=$ Medical Knowledge; $\mathrm{P}=$ Professional Practice

Note. $\#=$ frequency; $\mathrm{Mn}=$ Mean; $\mathrm{SD}=$ Standard Deviation; $\mathrm{SEM}=$ Standard Error Mean

A significant difference was not discovered between the two groups for any of the constructs: Medical Education, $t(41)=1.06, p=.29, p>.017$; Medical Knowledge, $t(41)=1.53, p=.13, p>.017$; and Professional Practice, $t$ $(41)=1.52, p=.14, p>.017$. There was not a significant relationship between completion of a specialization and self-reported competence across the three constructs.

\section{Demographic Variable Rotation Completion}

The $t$ - tests regarding rotation completion compared how pediatric residents perceived their average levels of self-reported competence in the three constructs regarding communication disorders and augmentative and assistive technology upon completion or non-completion of a specific rotation. Participants were coded as either 'Yes' or 'No' and the two groups will be compared on the average levels of self-reported competence in the three constructs regarding communication disorders and augmentative and assistive technology. 
Separate data was analyzed for the pediatric resident rotations deemed relevant to communication disorders and AAC. These included the following: adolescent medicine, ambulatory pediatrics, child development, community health programs, genetics, in- patient (wards), pediatric ICU, private practitioner's office and Weisskopf Children Evaluation Center (WCEC). For a complete listing of pediatric resident rotations and demographic changes across PLs see Table 7.

The ability of the data to meet methodological assumptions was independently addressed for each rotation. The assumptions for an independent $t$ test included independence, normality, and equality of variances.

Adolescent medicine. Adolescent Medicine's focus is for outpatient care of teenagers with a focus on understanding normal development and how various psychobiologic variations or diseases affect the overall health of the individual (University of Louisville, 2011).

Assumptions. The ability of the rotation data to meet methodological assumption was assessed. In this study, all observations were independent as each participant completed a single survey. Due to the robustness of a $t$-test, with two assumptions met, a histogram for the data points was not performed Levene's test gauged the homogeneity of variance among the variables. For all three constructs, the Levene's test was not significant at the .05 level: Medical Education (Levene's, $F=.00, p=.97, p>.05$ ), Medical Knowledge (Levene's, $F=.07, p=.80, p>.05$ ), Professional Practice (Levene's, $F=1.27, p$ $=.27, p>.05)$. There was not a violation of homogeneity, and the assumption for homogeneity of variance was met. 
Independent $t$ tests. The $t$ - test regarding adolescent rotation completion compared how pediatric residents perceived their average levels of self-reported competence in the three constructs regarding communication disorders and augmentative and assistive technology. Within the body of pediatric residents, the population having completed the adolescent medicine rotation totaled $30(69.8 \%)$. Those who indicated 'no' totaled $13(30.2 \%)$. .

Table 25 presents information regarding the frequency, mean, standard deviation and standard error of the mean for adolescent medicine rotation completion across the various constructs.

Table 25

Descriptive Statistics for Adolescent Medicine $(N=43)$

\begin{tabular}{|c|c|c|c|c|c|}
\hline DV & $A M$ & $\#$ & $\mathrm{Mn}$ & SD & SEM \\
\hline \multicolumn{6}{|l|}{$E$} \\
\hline & Y & 30 & 2.97 & .82 & .15 \\
\hline & $N$ & 13 & 2.20 & .95 & .26 \\
\hline \multicolumn{6}{|l|}{$\mathrm{K}$} \\
\hline & Y & 30 & 3.4 & 55 & .10 \\
\hline \multirow{3}{*}{$P$} & $N$ & 13 & 3.15 & .58 & .16 \\
\hline & $Y$ & 30 & 3.12 & .55 & .10 \\
\hline & $N$ & 13 & 2.92 & .73 & .20 \\
\hline
\end{tabular}

Note. $\mathrm{DV}=$ Dependent Variable $; \mathrm{AM}=$ Adolescent Medicine; $\mathrm{E}=$ Medical Education; $\mathrm{K}=$ Medical Knowledge; $\mathrm{P}=$ Professional Practice Note. $\#=$ Frequency; $\mathrm{Mn}=$ Mean; $\mathrm{SD}=$ Standard Deviation; $\mathrm{SEM}=$ Standard Error Mean

A significant difference was found between adolescent medicine rotation and one of the three constructs: Medical Education, $t(41)=-2.55, p=.015, p<$ .017 ; Medical Knowledge, $t(41)=-1.42, p=.16, p>.017$; and Professional Practice, $t(41)=-.99, p=.33, p>.017$. There was significant difference 
between completion of the adolescent medicine rotation and self-reported competence for Medical Education, but not Medical Knowledge or Professional Practice.

Ambulatory pediatrics. Within Ambulatory Pediatrics, residents are to provide preventive health care as well as management of acute and chronic illnesses. The focus of this rotation is the opportunity to develop skills in an outpatient setting (University of Louisville, 2011).

Assumptions. The ability of the rotation data to meet methodological assumption was assessed. In this study, all observations were independent as each participant completed a single survey. Due to the robustness of a $t$-test, with two assumptions met, a histogram for the data points was not performed.

Levene's test gauged the homogeneity of variance among the variables. For all three constructs, the Levene's test was not significant at the .05 level: Medical Education (Levene's, $F=1.01, p=.32, p>.05$ ), Medical Knowledge (Levene's, $F=.59, p=.45, p>.05$ ), Professional Practice (Levene's, $F=-.77, p$ $=.58, p>.05)$. There was not a violation of homogeneity, and the assumption for homogeneity of variance was met.

Independent $t$ tests. The $t$ - test regarding ambulatory pediatrics rotation completion compared how pediatric residents perceived their average levels of self-reported competence in the three constructs regarding communication disorders and augmentative and assistive technology. Within the body of pediatric residents, the population having completed the ambulatory pediatrics rotation totaled $17(40 \%)$. Those who indicated 'no' totaled $26(60 \%)$. 
Table 26 presents information regarding the frequency, mean, standard deviation and standard error of the mean for adolescent medicine rotation completion across the various constructs.

Table 26

Descriptive Statistics for Ambulatory Pediatrics $(N=43)$

\begin{tabular}{llllll} 
DV & AP & $\#$ & Mn & SD & SEM \\
\hline E & Y & 17 & 3.07 & .96 & .23 \\
& $\mathrm{~N}$ & 26 & 2.47 & .82 & .16 \\
$\mathrm{~K}$ & $\mathrm{Y}$ & 17 & 3.41 & .59 & .14 \\
& $\mathrm{~N}$ & 26 & 3.29 & .55 & .11 \\
$\mathrm{P}$ & $\mathrm{Y}$ & 17 & 3.15 & .61 & .12 \\
& $\mathrm{~N}$ & 26 & 3.00 & .61 & .12
\end{tabular}

Note. $\mathrm{DV}=$ Dependent Variable; Ambulatory Pediatrics; $\mathrm{E}=$ Medical Education; $\mathrm{K}=$ Medical Knowledge; $\mathrm{P}=$ Professional Practice Note. $\#$ = frequency; $\mathrm{Mn}=$ Mean; $\mathrm{SD}=$ Standard Deviation; $\mathrm{SEM}=$ Standard Error Mean

A significant difference was not found between the three groups: Medical Education, $t(41)=-2.20, p=.034, p>.017$; Medical Knowledge, $t(41)=-.70, p=$ $.50, p>.017$; and Professional Practice, $t(41)=-.77, p=.45, p>.017$. There was not a significant difference between completion of the ambulatory pediatrics rotation and self-reported competence for Medical Education, Medical Knowledge or Professional Practice.

\section{Child Development}

Assumptions. The ability of the rotation data to meet methodological assumption was assessed. In this study, all observations were independent as 
each participant completed a single survey. Due to the robustness of a $t$ - test, with two assumptions met, a histogram for the data points was not performed.

Levene's test gauged the homogeneity of variance among the variables.

For all three constructs, the Levene's test was not significant at the .05 level: Medical Education (Levene's, $F=.04, p=.85, p>.05$ ), Medical Knowledge (Levene's, $F=.00, p=.96, p>.05$ ), Professional Practice (Levene's, $F=2.46, p$ $=.12, p>.05)$. There was not a violation of homogeneity, and the assumption for homogeneity of variance was met.

Independent $t$ tests. The $t$ - test regarding child development rotation completion compared how pediatric residents perceived their average levels of self-reported competence in the three constructs regarding communication disorders and augmentative and assistive technology. Within the body of pediatric residents, the population having completed the child development rotation totaled $5(12 \%)$. Those who indicated 'no' totaled $38(88 \%)$.

Table 27 presents information regarding the frequency, mean, standard deviation and standard error of the mean for adolescent medicine rotation completion across the various constructs. 
Table 27

Descriptive Statistics for Child development $(N=43)$

\begin{tabular}{llllll} 
DV & CD & $\#$ & Mn & SD & SEM \\
\hline E & $Y$ & 5 & 3.44 & .96 & .43 \\
& $\mathrm{~N}$ & 38 & 2.61 & .88 & .14 \\
$\mathrm{~K}$ & $\mathrm{Y}$ & 5 & 3.10 & .60 & .27 \\
& $\mathrm{~N}$ & 38 & .34 & .56 & .09 \\
$\mathrm{P}$ & $\mathrm{Y}$ & 5 & 2.98 & .88 & .39 \\
& $\mathrm{~N}$ & 38 & 3.07 & .58 & .09 \\
& & & &
\end{tabular}

Note. $\mathrm{DV}=$ Dependent Variable $; \mathrm{CD}=$ Child Development; $\mathrm{E}=$ Medical Education; $\mathrm{K}=$ Medical Knowledge; $\mathrm{P}=$ Professional Practice Note. $\#=$ frequency; $\mathrm{Mn}=$ Mean; $\mathrm{SD}=$ Standard Deviation; $\mathrm{SEM}=$ Standard Error Mean

A significant difference was not found between any of the three groups: Medical Education, $t(41)=-1.97, p=.06, p>.017 ; \quad$ Medical Knowledge, $t(41)=$ $1.00, p=.32, p>.017$; and Professional Practice, $t(41)=.33, p=.75, p>.017$. There was not a significant difference between completion of the child development rotation and self-reported competence for Medical Education, Medical Knowledge or Professional Practice.

Community health programs. The goal of this rotation is to expose the resident to various community services and community settings that care for children (University of Louisville, 2011).

Assumptions. The ability of the rotation data to meet methodological assumption was assessed. In this study, all observations were independent as each participant completed a single survey. Due to the robustness of a $t$ - test, with two assumptions met, a histogram for the data points was not performed. 
Levene's test gauged the homogeneity of variance among the variables. For all three constructs, the Levene's test was not significant at the .05 level: Medical Education (Levene's, $F=.65, p=.42, p>.05$ ), Medical Knowledge (Levene's, $F=1.54, p=.22, p>.05$ ), Professional Practice (Levene's, $F=.00, p$ $=.97, p>.05)$. There was not a violation of homogeneity, and the assumption for homogeneity of variance was met.

Independent $t$ tests. The $t$ - test regarding community health programs rotation completion compared how pediatric residents perceived their average levels of self-reported competence in the three constructs regarding communication disorders and augmentative and assistive technology. Within the body of pediatric residents, the population having completed the community health programs rotation totaled $4(9 \%)$. Those who indicated 'no' totaled 39 $(91 \%)$.

Table 28 presents information regarding the frequency, mean, standard deviation and standard error of the mean for adolescent medicine rotation completion across the various constructs.

Table 28

Descriptive Statistics for Community Health Programs $(N=43)$

\begin{tabular}{llllll} 
DV & CHP & $\#$ & $M n$ & SD & SEM \\
\hline
\end{tabular}

$\mathrm{E}$

$\mathrm{K}$

$\begin{array}{lllll}\mathrm{Y} & 4 & 3.15 & 1.15 & .57 \\ \mathrm{~N} & 39 & 2.66 & .90 & .14 \\ \mathrm{Y} & & & & \\ \mathrm{N} & 4 & 3.75 & .40 & .20 \\ & 39 & 3.29 & .56 & .09\end{array}$

table continues on next page 
Table 28 continued

DV

CHP

\#

$\mathrm{Mn}$

SD

SEM

$\mathrm{P}$

$\begin{array}{lllll}Y & 4 & 3.51 & .56 & .28 \\ N & 39 & 3.02 & .60 & .10\end{array}$

Note. $\mathrm{DV}=$ Dependent Variable; $\mathrm{CHP}=$ Community Health Programs; $\mathrm{E}=$ Medical Education; $K=$ Medical Knowledge; $P=$ Professional Practice Note. $\#$ = frequency; $\mathrm{Mn}=$ Mean; $\mathrm{SD}=$ Standard Deviation; $\mathrm{SEM}=$ Standard Error Mean

A significant difference was not found between any of the three constructs and community health program rotation: Medical Education, $t(41)=-1.02, p=$ $.32, p>.017$; Medical Knowledge, $t(41)=-1.57, p=.13, p>.017$; and Professional Practice, $t(41)=-1.58, p=.12, p>.017$. There was not a significant difference between completion of the community health programs rotation and self-reported competence for Medical Education, Medical Knowledge or Professional Practice.

\section{Genetics}

Assumptions. The ability of the rotation data to meet methodological assumption was assessed. In this study, all observations were independent as each participant completed a single survey. Due to the robustness of a $t-t e s t$, with two assumptions met, a histogram for the data points was not performed.

Levene's test gauged the homogeneity of variance among the variables.

For all three constructs, the Levene's test was not significant at the .05 level: Medical Education (Levene's, $F=.06, p=.81, p>.05$ ), Medical Knowledge (Levene's, $F=.01, p=.94, p>.05$ ), Professional Practice (Levene's, $F=.62, p=$ 
$.42, p>.05)$. There was not a violation of homogeneity, and the assumption for homogeneity of variance was met.

Independent $t$ tests. These $t$-tests investigated possible differences between genetics rotation completion and pediatric residents' perceived competence on the three constructs. Within the body of pediatric residents, the population having completed the genetics rotation totaled $10(23 \%)$. Those who indicated 'no' totaled $33(77 \%)$.

Table 29X presents information regarding the frequency, mean, standard deviation and standard error of the mean for adolescent medicine rotation completion across the various constructs.

Table 29

Descriptive Statistics for Genetics ( $N=43)$

\begin{tabular}{llllll} 
DV & G & $\#$ & Mn & SD & SEM \\
\hline E & & & & & \\
& $Y$ & 10 & 3.00 & .88 &. \\
& $\mathrm{~N}$ & 33 & 2.62 & .91 & .16 \\
$\mathrm{~K}$ & $\mathrm{Y}$ & 10 & 3.43 & .56 & .18 \\
& $\mathrm{~N}$ & 33 & 3.31 & .60 & .10 \\
$\mathrm{P}$ & $\mathrm{Y}$ & 10 & 3.14 & .58 & .16 \\
& $\mathrm{~N}$ & 33 & 3.04 & .88 & .11
\end{tabular}

Note. $\mathrm{DV}=$ Dependent Variable $\mathrm{G}=$ Genetics; $\mathrm{E}=$ Medical Education; $\mathrm{K}=$ Medical Knowledge; $P=$ Professional Practice

Note. $\#=$ Frequency; $\mathrm{Mn}=$ Mean; $\mathrm{SD}=$ Standard Deviation; $\mathrm{SEM}=$ Standard Error Mean

A significant difference not was found between any of the three groups: Medical Education, $t(41)=-1.16, p=.25, \mathrm{p}>.017$; Medical Knowledge, $t(41)=-$ $.61, p=.54, p>.017 ;$ and Professional Practice, $t(41)=-.45, p=.65, p>.017$ 
There was not a significant difference between completion of the genetics rotation and self-reported competence for Medical Education, Medical Knowledge or Professional Practice.

In-patient Service (Wards).

This rotation focuses on general inpatient medicine. The residents are responsible for the inpatient management of all the general medical patients admitted to Kosair Children's Hospital (University of Louisville, 2011).

Assumptions. The ability of the rotation data to meet methodological assumption was assessed. In this study, all observations were independent as each participant completed a single survey. Due to the robustness of a $t-$ test, with two assumptions met, a histogram for the data points was not performed.

Levene's test gauged the homogeneity of variance among the variables. For all three constructs, the Levene's test was not significant at the .05 level: Medical Education (Levene's, $F=.126, p=.725, p>.05$ ), Medical Knowledge (Levene's, $F=2.276, p=.139, p>.05$ ), Professional Practice (Levene's, $F=$ $.211, p=.648, p>.05)$. There was not a violation of homogeneity, and the assumption for homogeneity of variance was met.

Independent $t$ tests. These $t$ - tests investigated possible differences between In-Patient Services (Wards) rotation completion across pediatric residents' perceived competence on the three constructs. Within the body of pediatric residents, the population having completed the In-Patient Service (Wards) rotation totaled $38(88 \%)$. Those who indicated 'no' totaled $5(12 \%)$. 
Table 30 presents information regarding the frequency, mean, standard deviation and standard error of the mean for In-Patient Service (Wards) rotation completion across the various constructs.

Table 30

Descriptive Statistics for In-Patient Service (Wards) $(N=43)$

\begin{tabular}{llllll} 
DV & IPS & $\#$ & Mn & SD & SEM \\
\hline $\mathrm{E}$ & $\mathrm{Y}$ & 38 & 2.79 & .86 & .14 \\
& $\mathrm{~N}$ & 5 & 2.08 & 1.19 & .53 \\
$\mathrm{~K}$ & $\mathrm{Y}$ & 38 & 3.34 & .58 & .09 \\
& $\mathrm{~N}$ & 5 & 3.30 & .42 & .19 \\
$\mathrm{P}$ & $\mathrm{Y}$ & 38 & 3.08 & .61 & .10 \\
& $\mathrm{~N}$ & 5 & 2.91 & .69 & .31
\end{tabular}

Note. $\mathrm{DV}=$ Dependent Variable; IPS $=$ In - Patient Services; $\mathrm{E}=$ Medical Education; $K=$ Medical Knowledge; $P=$ Professional Practice Note. $\#=$ Frequency; $\mathrm{Mn}=$ Mean; $\mathrm{SD}=$ Standard Deviation; $\mathrm{SEM}=$ Standard Error Mean

A significant difference not was found between any of the three groups:

Medical Education $=.73, t(41)=-1.66, p>.017 ;$ Medical Knowledge $=.14, t(41)$ $=-.12, p>.017 ;$ and Professional Practice $=.65, t(41)=-.54, p>.017$. There was not a significant difference between completion of the In-Patient Service rotation and self-reported competence for Medical Education, Medical Knowledge or Professional Practice.

Pediatric Intensive Care Unit.

This rotation provides direct involvement in the management of critically ill and injured children (University of Louisville, 2011). 
Assumptions. The ability of the rotation data to meet methodological assumption was assessed. In this study, all observations were independent as each participant completed a single survey. Due to the robustness of a $t-t e s t$, with two assumptions met, a histogram for the data points was not performed.

Levene's test gauged the homogeneity of variance among the variables. For all three constructs, the Levene's test was not significant at the .05 level: Medical Education (Levene's, $F=.38, p=.54, p>.05$ ), Medical Knowledge (Levene's, $F=.28, p=.60, p>.05$ ), Professional Practice (Levene's, $F=.46, p=$ $.50, p>.05)$. There was not a violation of homogeneity, and the assumption for homogeneity of variance was met.

Independent $t$ tests. These $t$-tests investigated possible differences between Pediatric Intensive Care Unit rotation completion across pediatric residents' perceived competence on the three constructs. Within the body of pediatric residents, the population having completed the Pediatric Intensive Care Unit rotation totaled $16(37 \%)$. Those who indicated 'no' totaled $27(63 \%)$.

Table 31 presents information regarding the frequency, mean, standard deviation and standard error of the mean for adolescent medicine rotation completion across the various constructs. 
Table 31

Descriptive Statistics for Pediatric Intensive Care Unit $(N=43)$

\begin{tabular}{lccccc} 
DV & PICU & $\#$ & Mn & SD & SEM \\
\hline E & Y & 16 & 3.31 & .87 & .22 \\
& $N$ & 27 & 2.35 & .75 & .14 \\
$\mathrm{~K}$ & $\mathrm{~N}$ & 16 & 3.58 & .51 & .13 \\
& $\mathrm{~N}$ & 27 & 3.19 & .55 & .11 \\
$\mathrm{P}$ & $\mathrm{Y}$ & 16 & 3.31 & .62 & .16 \\
& $\mathrm{~N}$ & 27 & 2.92 & .56 & .11
\end{tabular}

Note. $\mathrm{DV}=$ Dependent Variable; $\mathrm{E}=$ Medical Education; $\mathrm{K}=$ Medical Knowledge; $P=$ Professional Practice

Note. $\#=$ Frequency; $\mathrm{Mn}=$ Mean; $\mathrm{SD}=$ Standard Deviation; $\mathrm{SEM}=\mathrm{Standard}$ Error Mean

A significant difference was found between one of the three groups:

Medical Education, $t(41)=-3.84, p=.00, p<.017 ;$ Medical Knowledge, $t(41)=-$ 2.32, $p=.03, p>.017$; and Professional Practice, $t(41)=-2.09, p=.04, p>$ .017. A significant difference was found between completion of Pediatric Intensive Care Unit rotation and self-reported competence for Medical Education but not Medical Knowledge or Professional Practice.

\section{Private Practitioner's Office}

All residents complete this rotation for one month in which they see and care for patients in a private practice setting in the community (University of Louisville, 2011).

Assumptions. The ability of the rotation data to meet methodological assumption was assessed. In this study, all observations were independent as 
each participant completed a single survey. Due to the robustness of a $t-t e s t$, with two assumptions met, a histogram for the data points was not performed.

Levene's test gauged the homogeneity of variance among the variables. For all three constructs, the Levene's test was not significant at the .05 level: Medical Education (Levene's, $F=.01, p=.93, p>.05$ ), Medical Knowledge (Levene's, $F=.27, p=.61, p>.05$ ), Professional Practice (Levene's, $F=.02, p=$ $.88, p>.05)$. There was not a violation of homogeneity, and the assumption for homogeneity of variance was met.

Independent $t$ tests. These $t-$ tests investigated possible differences between Private Practitioner's Office rotation completion across pediatric residents' perceived competence on the three constructs. Within the body of pediatric residents, the population having completed the Private Practitioner's Office rotation totaled $12(28 \%)$. Those who indicated 'no' totaled $31(72 \%)$.

Table 32 resents information regarding the frequency, mean, standard deviation and standard error of the mean for adolescent medicine rotation completion across the various constructs.

Table 32

Descriptive Statistics for Private Practitioner's Office $(N=43)$

\begin{tabular}{llllll} 
DV & PPO & $\#$ & Mn & SD & SEM \\
\hline $\mathrm{E}$ & $\mathrm{Y}$ & 12 & 2.78 & .93 & .27 \\
& $\mathrm{~N}$ & 31 & 2.68 & .92 & .17 \\
$\mathrm{~K}$ & $\mathrm{Y}$ & 12 & 3.32 & .64 & .18 \\
& $\mathrm{~N}$ & 31 & 3.34 & .54 & .10 \\
& & \multicolumn{5}{c}{ table continues on next page }
\end{tabular}


Table 32 continues

\begin{tabular}{lccccc} 
DV & PPO & $\#$ & Mn & SD & SEM \\
\hline P & & & & & \\
& Y & 12 & 3.05 & .60 & .17 \\
& $N$ & 31 & 3.07 & .62 & .11
\end{tabular}

Note. $\mathrm{DV}=$ Dependent Variable $\mathrm{PPO}=$ Private Practitioners Office $; \mathrm{E}=$ Medical Education; $\mathrm{K}=$ Medical Knowledge; $\mathrm{P}=$ Professional Practice Note. $\#=$ Frequency; $\mathrm{Mn}=$ Mean; $\mathrm{SD}=$ Standard Deviation; $\mathrm{SEM}=$ Standard Error Mean

A significant difference not was found between any of the three groups: Medical Education, $t(41)=-.34, p=.74, p>.017$; Medical Knowledge, $t(41)=$ $.13, p=.90, p>.017 ;$ and Professional Practice, $t(41)=.10, p=.92, p>.017$. There was not a significant difference between completion of the Private Practitioner's Office rotation and self-reported competence for Medical Education, Medical Knowledge or Professional Practice.

\section{Weisskopf Child Evaluation Center.}

Participation in this rotation allows for observation and understanding of a wide variety of developmental and genetic disabilities. Residents learn about the assessment and treatment of disabilities (University of Louisville, 2011).

Assumptions. The ability of the rotation data to meet methodological assumption was assessed. In this study, all observations were independent as each participant completed a single survey. Due to the robustness of a $t$-test, with two assumptions met, a histogram for the data points was not performed.

Levene's test gauged the homogeneity of variance among the variables.

For all three constructs, the Levene's test was not significant at the .05 level: 
Medical Education (Levene's, $F=3.40, p=.07, p>.05$ ), Medical Knowledge (Levene's, $F=.07, p=.80, p>.05$ ), Professional Practice (Levene's, $F=.91, p=$ $.35, p>.05)$. There was not a violation of homogeneity, and the assumption for homogeneity of variance was met.

Independent $t$ tests. These $t$ - tests investigated possible differences between WCEC rotation completion across pediatric residents' perceived competence on the three constructs. Within the body of pediatric residents, the population having completed the WCEC rotation totaled $5(12 \%)$. Those who indicated 'no' totaled $38(88 \%)$.

Table 33 presents information regarding the frequency, mean, standard deviation and standard error of the mean for adolescent medicine rotation completion across the various constructs.

Table 33

Descriptive Statistics for WCEC $(N=43)$

DV WCEC

$\mathrm{Mn}$

SD SEM

$E$

$\mathrm{K}$

$\begin{array}{lllll}Y & 5 & 4.08 & .44 & .20 \\ \mathrm{~N} & 38 & 2.54 & .80 & .13\end{array}$

$\begin{array}{lllll}Y & 5 & 3.50 & .63 & .28 \\ N & 38 & 3.32 & .56 & .09\end{array}$

$\mathrm{P}$

$\begin{array}{lllll}\mathrm{Y} & 5 & 3.41 & .71 & .32 \\ \mathrm{~N} & 38 & 3.02 & .59 & .10\end{array}$

Note. $\mathrm{E}=$ Medical Education; $\mathrm{K}=$ Medical Knowledge; $\mathrm{P}=$ Professional Practice Note. $\#=$ Frequency; $\mathrm{Mn}=$ Mean; $\mathrm{SD}=$ Standard Deviation; $\mathrm{SEM}=$ Standard Error Mean

A significant difference was found between one of the three groups: 
Medical Education, $t(41)=-4.21, p=.00, p<.05 ;$ Medical Knowledge, $t(41)=-$ $.54, p=.59, p>.017$; and Professional Practice, $t(41)=-1.37, p=.18, p>.017$. There was significant difference between completion of the WCEC rotation and self-reported competence for Medical Education, but not Medical Knowledge or Professional Practice.

\section{Demographic Variable Educational Methods}

Another demographic variable analyzed within the data was educational methods. The independent variable, educational methods were coded into two groups, 'Yes' or 'No'. The two groups (Yes or No) were compared for mean differences on the average levels of self-reported competence in the three constructs regarding communication disorders and augmentative and assistive technology. The initial question posed was directed to the occurrence of any educational experiences regarding communication disorders or AAC within a rotation. The rest of the analysis that follows addresses specific formats were these learning experiences may have occurred. Educational methods will include one or more of the following: ambulatory pediatric rotation, subspecialty rotation, morning report, core conference, didactic or board conference.

Education: Rotation. The initial question posed was directed to the occurrence of any educational experiences regarding communication disorders or AAC within a rotation. The two groups ('Yes' or 'No') were compared for mean differences on the average levels of self-reported competence in the three constructs regarding communication disorders and augmentative and assistive technology. 
Assumptions. The ability of the data to meet methodological assumption was assessed. The assumptions for an independent $t$ test included independence, normality, and equality of variances. In this study, all observations were independent as each participant completed a single survey. Due to the robustness of a $t$-test, with two assumptions met, a histogram for the data points was not performed.

Levene's test gauged the homogeneity of variance among the variables. For all three constructs, the Levene's test was not significant at the .05 level: For communication disorders: Medical Education (Levene's, $F=.00, p=.10, p>$ .05 ), Medical Knowledge (Levene's, $F=.22, p=.65, p>.05$ ), Professional Practice (Levene's, $F=2.79, p=.10, p>.05$ ); For AAC: Medical Education (Levene's, $F=2.24, p=.14, p>.05$ ), Medical Knowledge (Levene's, $F=.03, p=$ $.86, p>.05$ ), Professional Practice (Levene's, $F=.28, p=.60, p>.05$ ). There was not a violation of homogeneity across either data sets, and the assumption for homogeneity of variance was met for both.

Independent $t$ tests. The $t$ - tests investigated differences between education during a rotation and the three constructs. Within the body of pediatric residents, the population reporting direct education within a rotation regarding communication disorders totaled $7(16 \%)$. Those who indicated 'no' totaled 36 $(84 \%)$. Within the body of pediatric residents, the population reporting direct education within a rotation regarding AAC totaled $3(7 \%)$. Those who indicated 'no' totaled $40(93 \%)$. For information regarding those specific specializations, see Tables 3 and 4. Table 34 presents information regarding the frequency, 
mean, standard deviation and standard error of the mean for gender across the various constructs.

Table 34

Descriptive Statistics for Education: Rotation ( $N=43)$

$\underline{\text { IV }}$

$C D$

E

Y $\quad 7$

AAC
$\mathrm{N}$

$\mathrm{K}$

$\mathrm{Y} \quad 7$

$\mathrm{P}$

N 36

Y $\quad 7$

\section{7}

7

36

7

36

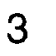

40

K

$\mathrm{N}$

$Y$

$\mathrm{N}$

P

E
3

40

3

40
$\mathrm{Mn}$

3.43

2.60

3.33

3.34

3.18

3.04
SD

SEM

$\begin{array}{ll}Y & 3 \\ N & 40\end{array}$

3.87

2.62

3.17

3.35

3.08

3.06
.91

.86

.63

.56

.83

.57

.42

.89

.67

.56

.75

.61
.34

.14

.24

.09

.31

.09

Note. $\mathrm{E}=$ Medical Education; $\mathrm{K}=$ Medical Knowledge; $\mathrm{P}=$ Professional Practice Note. $\quad \#=$ Frequency; $\mathrm{Mn}=$ Mean; $\mathrm{SD}=$ Standard Deviation; $\mathrm{SEM}=$ Standard Error Mean

Note. IV = Independent Variable; DV = Dependent Variable; E:R Education: Rotation

For communication disorders a significant difference was not found between the means within data sets: Medical Education, $t(41)=-2.40, p=.02, p$ $>.017$; Medical Knowledge, $t(41)=.020, p=.98, p>.017$; and Professional Practice, $t(41)=-.42, p=.59, p>.017$. There was not a significant relationship between any data sets for communication disorders. 
For AAC a significant difference was not found between the means within data sets: Medical Education, $t(41)=-2.37, p=.02, p>.017$; Medical Knowledge, $t(41)=.54, p=.59, p>.017$; and Professional Practice, $t(41)=-$ $.06, p=.95, p>.017$. There were not any significant relationships between data sets and the presentation of AAC educational experiences within a rotation.

Education: Ambulatory pediatric rotation. The next focus of data analysis was to assess is any specific forms of educational experiences presented a significant difference if any within perceived competency for the three constructs. The two groups ('Yes' or 'No' regarding education of communication disorders and AAC during Ambulatory Pediatric Rotation) were compared for mean differences on the average levels of self-reported competence across the three constructs.

Assumptions. The ability of the data to meet methodological assumption was assessed. The assumptions for an independent $t$ test included independence, normality, and equality of variances. In this study, all observations were independent as each participant completed a single survey. Due to the robustness of a $t$ - test, with two assumptions met, a histogram for the communication disorder data points was not performed.

Levene's test gauged the homogeneity of variance among the variables. Due to the significant difference between the For all three constructs, the Levene's test was not significant at the .05 level: For communication disorders: Medical Education (Levene's, $F=.11, p=.74, p>.05$ ), Medical Knowledge (Levene's, $F=6.37, p=.02, p>.05$ ), Professional Practice (Levene's, $F=.61$, 
$p=.44, p>.05)$; For AAC Levene's was unable to be completed due to insufficient participants. There was a violation of homogeneity for communication disorders, and the assumption for homogeneity of variance was met. AAC was unable to be assessed.

Independent $t$ tests. The $t$ - tests investigated differences between education during a rotation and the three constructs. Within the body of pediatric residents, the population reporting direct education within a rotation regarding communication disorders totaled $5(12 \%)$. Those who indicated 'no' totaled 38 $(88 \%)$. Within the body of pediatric residents, the population reporting direct education within a rotation regarding AAC totaled $1(2 \%)$. Those who indicated 'no' totaled $42(98 \%)$. Table 35 presents information regarding the frequency, mean, standard deviation and standard error of the mean for gender across the various constructs.

Table 35

Descriptive Statistics for Education: Ambulatory Pediatric Rotation $(N=43)$

DV E:APR $\quad \# \quad$ Mn $\quad$ SD $\quad$ SEM

\begin{tabular}{llllll}
\hline $\mathrm{E}$ & $\mathrm{Y}$ & 5 & 3.56 & .82 & .37 \\
& $\mathrm{~N}$ & 38 & 2.59 & .88 & .14 \\
$\mathrm{~K}$ & $\mathrm{Y}$ & 5 & 3.83 & .17 & .07 \\
& $\mathrm{~N}$ & 38 & 3.27 & .56 & .09 \\
$\mathrm{P}$ & $\mathrm{Y}$ & 5 & 3.45 & .42 & .19 \\
& $\mathrm{~N}$ & 38 & 3.01 & .62 & .10 \\
& & & &
\end{tabular}

Note. $\mathrm{E}=$ Medical Education; $\mathrm{K}=$ Medical Knowledge; $\mathrm{P}=$ Professional Practice Note. $\mathrm{DV}=$ Dependent Variable; E:APR Education: Ambulatory Pediatric Rotation 
For communication disorders a significant difference was found between the means within data set and not for the other two: Medical Education, $t(41)=$ 2.33, $p=.025, p<.05 ;$ Medical Knowledge, $t(41)=-2.19, p=.03, p<.05 ;$ and Professional Practice, $t(41)=.08, p=.13, p>.017$. There was a significant relationship between both Medical Education and Medical Knowledge and the presentation of educational experiences regarding communication disorders within the ambulatory pediatric rotation. A significant relationship was not found between professional practice and the presentation of educational experiences within ambulatory pediatric rotations regarding communication disorders.

The analysis for AAC was unable to be completed due to the limited population that had this form of educational experience, 1 . To make any inferences from this minimal data set would not be representative of the overall population.

Education: Subspecialty rotation. Subspecialty rotation was the next educational format to be investigated. The two groups ('Yes' or 'No' regarding education of communication disorders and AAC during Subspecialty Rotation) were compared for mean differences on the average levels of self-reported competence across the three constructs.

Assumptions. The ability of the data to meet methodological assumption was assessed. The assumptions for an independent $t$ test included independence, normality, and equality of variances. In this study, all observations were independent as each participant completed a single survey. 
Due to the robustness of a $t$ - test, with two assumptions met, a histogram for the communication disorder data points was not performed.

Levene's test gauged the homogeneity of variance among the variables. For all three constructs, the Levene's test was not significant at the .05 level: For communication disorders: Medical Education (Levene's, , $F=.93, p=.34, p>$ .05 ), Medical Knowledge (Levene's, $F=1.212, p=.277, p>.05$ ), Professional Practice (Levene's, $F=1.13, p=.29, p>.05$ ); For AAC: Education (Levene's, $F$ $=.50, p=.49, p>.05$ ), Medical Knowledge (Levene's, $F=4.52, p=.04, p<.05$ ), Professional Practice (Levene's, $F=1.64, p=.21, p>.05$ ). There was not a violation of homogeneity for communication disorders, and the assumption for homogeneity of variance was met. For AAC Levene's was unable to be completed due to insufficient participants. There was a violation of homogeneity for communication disorders, and the assumption for homogeneity of variance was met. AAC was unable to be assessed.

Independent $t$ tests. The $t$-tests investigated differences between education during a Subspecialized Rotation and the three constructs. Within the body of pediatric residents, the population reporting direct education within a Subspecialty Rotation regarding communication disorders totaled 5 (12\%). Those who indicated 'no' totaled $38(88 \%)$. Within the body of pediatric residents, the population reporting direct education within a Subspecialty Rotation regarding AAC totaled $3(7 \%)$. Those who indicated 'no' totaled 40 (93\%). Table 36 presents information regarding the frequency, mean, standard deviation and standard error of the mean for gender across the various 
constructs. Data regarding AAC was not presented due to the limited sample size and violation of homogeneity of variance.

Table 36

Descriptive Statistics for Education: Subspecialized Rotation $(N=43)$

DV

E:SR

$\mathrm{Mn}$

SD

SEM

\begin{tabular}{llllll}
\hline $\mathrm{E}$ & $\mathrm{Y}$ & 5 & 3.2800 & .81976 & .36661 \\
& $\mathrm{~N}$ & 38 & 2.6316 & .91182 & .14792 \\
$\mathrm{~K}$ & $\mathrm{Y}$ & 5 & 3.7333 & .43461 & .19437 \\
& $\mathrm{~N}$ & 38 & 3.2851 & .56141 & .09107 \\
$\mathrm{P}$ & $\mathrm{Y}$ & & & & \\
& $\mathrm{N}$ & 3 & 3.4700 & .35637 & .15937 \\
& & 38 & 3.0090 & .61806 & .10026
\end{tabular}

Note. $\mathrm{E}=$ Medical Education; $\mathrm{K}=$ Medical Knowledge; $\mathrm{P}=$ Professional Practice Note. IV = Independent Variable; DV = Dependent Variable; E:SR Education: Subspecialized Rotation

For communication disorders a significant difference was not found between the means within data sets: Medical Education, $t(41)=-1.509, p=$ $.129, p>.017$; Medical Knowledge, $t(41)=-1.712, p=.094, p>.017$; and Professional Practice, $t(41)=-1.622, p=.113, p>.017$. A significant relationship was not found between Medical Education, Medical Knowledge or Professional Practice and the presentation of educational experiences regarding communication disorders within the Subspecialized Rotation.

The analysis for AAC was unable to be completed due to the limited population that had this form of educational experience, 1. To make any inferences from this minimal data set would not be representative of the overall population, especially in light of this comparison's violation of homogeneity of variance. 
Education: Morning report. Morning reports were the next educational format to be investigated. This daily session offers a format for residents to discuss with the faculty newly admitted patients and follow-up of interesting and complex cases. It gives the residents a perspective on the variety of illnesses in the hospital each day and the opportunity to discuss in-depth specific educational topics (University of Louisville, 2011). The two groups ('Yes' or 'No' regarding morning report discussion of communication disorders and AAC) were compared for mean differences on the average levels of self-reported competence across the three constructs.

Assumptions. The ability of the data to meet methodological assumption was assessed. The assumptions for an independent $t$ test included independence, normality, and equality of variances. In this study, all observations were independent as each participant completed a single survey. Due to the robustness of a $t$ - test, with two assumptions met, a histogram for the communication disorder data points was not performed.

Levene's test gauged the homogeneity of variance among the variables. For all three constructs, the Levene's test was not significant at the .05 level: For communication disorders: Medical Education (Levene's, $F=2.222, p=.144, p>$ .05 ), Medical Knowledge (Levene's, $F=1.187, p=.282, p>.05$ ), Professional Practice (Levene's, $F=.097, p=.757, p>.05$ ); For AAC Levene's was unable to be completed due to insufficient participants. There was a violation of homogeneity for communication disorders, and the assumption for homogeneity of variance was met. AAC was unable to be assessed. 
Independent $t$ tests. The $t-$ tests investigated differences between education during Morning Reports and the three constructs. Within the body of pediatric residents, the population reporting direct education within a Morning Report regarding communication disorders totaled $5(12 \%)$. Those who indicated 'no' totaled $38(88 \%)$. Within the body of pediatric residents, the population reporting direct education within a Morning Report regarding AAC totaled $3(7 \%)$. Those who indicated 'no' totaled $40(93 \%)$. Table 37 presents information regarding the frequency, mean, standard deviation and standard error of the mean for gender across the various constructs. Data regarding $A A C$ was not presented due to the limited sample size and violation of homogeneity of variance.

Table 37

Descriptive Statistics for Education: Morning Report $(N=43)$

\begin{tabular}{llllll} 
DV & E:MR & $\#$ & Mn & SD & SEM \\
\hline $\mathrm{E}$ & $\mathrm{Y}$ & 5 & 3.4000 & .58878 & .29439 \\
& $\mathrm{~N}$ & 38 & 2.6359 & .91981 & .14729 \\
$\mathrm{~K}$ & $\mathrm{Y}$ & 5 & 3.6250 & .43833 & .21916 \\
& $\mathrm{~N}$ & 38 & 3.3077 & .57039 & .09134 \\
$\mathrm{P}$ & $\mathrm{Y}$ & & & & \\
& $\mathrm{N}$ & 38 & 3.5375 & .53131 & .26566 \\
& & 3.0139 & .60130 & .09629
\end{tabular}

Note. $\mathrm{E}=$ Medical Education; $\mathrm{K}=$ Medical Knowledge; $\mathrm{P}=$ Professional Practice Note. IV = Independent Variable; DV = Dependent Variable; E:MR = Education: Morning Report

For communication disorders a significant difference was not found between the means within data sets: Medical Education, $t(41)=-1.618, p=$ $.113, p>.017 ;$ Medical Knowledge, $t(41)=-1.076, p=.288, p>.017$; and 
Professional Practice, $t(41)=-1.853, p=.102, p>.017$. A significant relationship was not found between Medical Education, Medical Knowledge or Professional Practice and the presentation of educational experiences regarding communication disorders within the Morning Reports.

The analysis for AAC was unable to be completed due to the limited population that had this form of educational experience, 3. To make any inferences from this minimal data set would not be representative of the overall population, especially in light of this comparison's violation of homogeneity of variance.

Education: Core conference. Core conferences were the next educational format to be investigated. These conferences provide residents with comprehensive core curriculum of pediatric topics as determined by the chief residents and program director (University of Louisville, 2011). The two groups ('Yes' or 'No' regarding morning report discussion of communication disorders and $A A C$ ) were compared for mean differences on the average levels of selfreported competence across the three constructs.

Assumptions. The ability of the data to meet methodological assumption was assessed. The assumptions for an independent $t$ test included independence, normality, and equality of variances. In this study, all observations were independent as each participant completed a single survey. Due to the robustness of a $t$ - test, with two assumptions met, a histogram for the communication disorder data points was not performed. 
Levene's test gauged the homogeneity of variance among the variables. For all three constructs, the Levene's test was not significant at the .05 level: For communication disorders: Medical Education (Levene's, $F=.986, p=.326, p>$ $.05)$, Medical Knowledge (Levene's, $F=.813, p=.373, p>.05$ ), Professional Practice (Levene's, $F=.477, p=.494, p>.05$ ). For AAC Levene's was unable to be completed due to insufficient participants. There was a violation of homogeneity for communication disorders, and the assumption for homogeneity of variance was met. AAC was unable to be assessed.

Independent $t$ tests. The $t$ - tests investigated differences between education during Core Conferences and the three constructs. Within the body of pediatric residents, the population reporting direct education within a Core Conference regarding communication disorders totaled 7 (16\%). Those who indicated 'no' totaled $36(84 \%)$. Within the body of pediatric residents, the population reporting direct education within a Core Conference regarding AAC totaled $3(7 \%)$. Those who indicated 'no' totaled $40(93 \%)$. Table 38 presents information regarding the frequency, mean, standard deviation and standard error of the mean for gender across the various constructs. 
Table 38

Descriptive Statistics for Education: Core Conference $(N=43)$

\begin{tabular}{llllll} 
DV & $\mathrm{E}: \mathrm{CC}$ & $\#$ & $M n$ & SD & SEM \\
\hline $\mathrm{E}$ & $\mathrm{Y}$ & 7 & 3.0857 & .77337 & .29230 \\
& $\mathrm{~N}$ & 36 & 2.6333 & .93381 & .15563 \\
$\mathrm{~K}$ & $\mathrm{Y}$ & 7 & 3.6429 & .45571 & .17224 \\
& $\mathrm{~N}$ & 36 & 3.2778 & .56765 & .09461 \\
$\mathrm{P}$ & $\mathrm{Y}$ & 7 & 3.2323 & .47636 & .18005 \\
& $\mathrm{~N}$ & 36 & 3.0296 & .63197 & .10533
\end{tabular}

Note. $\mathrm{E}=$ Medical Education; $\mathrm{K}=$ Medical Knowledge; $\mathrm{P}=$ Professional Practice Note. IV = Independent Variable; $\mathrm{DV}=$ Dependent Variable; $\mathrm{E}: \mathrm{CC}=$ Education: Core Conference

For communication disorders a significant difference was not found between the means within data sets: Medical Education, $t(41)=-1.201, p=$ .237, $p$ >.017; Medical Knowledge, $t(41)=-1.599, p=.117, p>.017$; and Professional Practice, $t(41)=-.802, p=.427, p>.017$. A significant relationship was not found between Medical Education, Medical Knowledge or Professional Practice and the presentation of educational experiences regarding communication disorders within core conferences.

The analysis for AAC was unable to be completed due to the limited population that had this form of educational experience, 3. To make any inferences from this minimal data set would not be representative of the overall population, especially in light of this comparison's violation of homogeneity of variance. 
Education: Didactic. Didactics were the next educational format to be investigated. The two groups ('Yes' or 'No' regarding morning report discussion of communication disorders and $\mathrm{AAC}$ ) were compared for mean differences on the average levels of self-reported competence across the three constructs.

Assumptions. The ability of the data to meet methodological assumption was assessed. The assumptions for an independent $t$ test included independence, normality, and equality of variances. In this study, all observations were independent as each participant completed a single survey. Due to the robustness of a $t$-test, with two assumptions met, a histogram for the communication disorder data points was not performed.

Levene's test gauged the homogeneity of variance among the variables. For all three constructs, the Levene's test was not significant at the .05 level: For communication disorders: Medical Education (Levene's, $F=.641, p=.428, p>$ .05 ), Medical Knowledge (Levene's, $F=2.650, p=.111, p>.05$ ), Professional Practice (Levene's, $F=.041, p=.840, p>.05$ ). For AAC Levene's was unable to be completed due to insufficient participants. There was a violation of homogeneity for communication disorders, and the assumption for homogeneity of variance was met. AAC was unable to be assessed.

Independent $t$ tests. The $t$ - tests investigated differences between education during Didactics and the three constructs. Within the body of pediatric residents, the population reporting direct education within a Didactic regarding communication disorders totaled $7(16 \%)$. Those who indicated 'no' totaled 33 $(84 \%)$. Within the body of pediatric residents, the population reporting direct 
education within a rotation regarding AAC totaled $3(7 \%)$. Those who indicated 'no' totaled $40(93 \%)$. Table 39 presents information regarding the frequency, mean, standard deviation and standard error of the mean for gender across the various constructs.

Table 39

Descriptive Statistics for Education: Didactics $(N=43)$

\begin{tabular}{llllll} 
DV & E:D & $\#$ & $M n$ & SD & SEM \\
\hline $\mathrm{E}$ & $\mathrm{Y}$ & 7 & 3.5143 & .81533 & .30817 \\
& $\mathrm{~N}$ & 36 & 2.5500 & .85973 & .14329 \\
$\mathrm{~K}$ & $\mathrm{Y}$ & 7 & 3.7381 & .37090 & .14019 \\
& $\mathrm{~N}$ & 36 & 3.2593 & .56406 & .09401 \\
$\mathrm{P}$ & $\mathrm{Y}$ & 7 & 3.6808 & .54398 & .20560 \\
& $\mathrm{~N}$ & 36 & 2.9424 & .55058 & .09176
\end{tabular}

Note. $\mathrm{E}=$ Medical Education; $\mathrm{K}=$ Medical Knowledge; $\mathrm{P}=$ Professional Practice Note. IV = Independent Variable; DV = Dependent Variable; E:D = Education: Didactic

For communication disorders a significant difference was found between the means of two of the three constructs within data sets: Medical Education, $t$ $(41)=-2.735, p=.009, p<017 ;$ Medical Knowledge, $t(41)=-2.146, p=.038, p$ $>.017$; and Professional Practice, $t(41)=-3.252, p=.002, p<.017$. A significant relationship was found between Medical Education, and Professional Practice and the presentation of educational experiences regarding communication disorders within Didactics. A significant relationship was not found for the construct of Medical Knowledge and educational experiences. 
The analysis for AAC was unable to be completed due to the limited population that had this form of educational experience, 3. To make any inferences from this minimal data set would not be representative of the overall population, especially in light of this comparison's violation of homogeneity of variance.

Education: Board conference. Board Conferences were the next educational format to be investigated. For Board Conferences, once a month a different topic is presented (University of Louisville, 2011). Residents have articles to read on the monthly topic (University of Louisville, 2011). The two groups ('Yes' or 'No' regarding morning report discussion of communication disorders and $A A C$ ) were compared for mean differences on the average levels of self-reported competence across the three constructs.

In this study analysis of assumptions, Levenes' was found significant for both communication disorders and AAC. Further $t$ - test assessment could not be conducted due to a limited sample population. 


\section{Appendix D}

Survey of Pediatric Residents:

Communication Disorders and

Augmentative and Alternative Communication (AAC):

Final Expert Panel Copy

Preamble 


\section{Survey of Pediatric Residents: \\ Communication Disorders and \\ Augmentative and Alternative Communication (AAC): \\ Final Expert Panel Copy \\ Preamble}

\section{Dear Pediatric Resident:}

You are being invited to participate in a research study by participating in a survey to examine your perceptions of your competence in relation to Communication Disorders and AAC.

Pediatricians, the American Academy of Pediatrics and the federal government believe that pediatricians should have a significant role in the provision and supervision of therapies and Augmentative and Alternative Communication (AAC) (Sneed, et al. 2004). Within their practice, pediatricians are to act as a medical home for children and take on the ethical responsibility to coordinate the care of a child up to age twenty-one (Brewer, McPherson, Magrab, \& Hutchins, 1989; American Academy of Pediatrics, 2005;American Academy of Pediatrics, 1999). Pediatricians must have knowledge of many aspects of a child's development or lack thereof, including a potential need for speechlanguage therapy and augmentative and alternative communication (AAC).

- Communication Disorders are impairments in an individual's "ability to receive, send, process, and comprehend concepts or verbal, nonverbal and graphic symbol systems" (American Speech-Language-Hearing Association, 1993).

- Augmentative and alternative communication (AAC) is an area of clinical and educational practice that offers a set of strategies and approaches to supplement or replace natural speech and/or handwriting.

- Competence within this survey is framed by the ACGME guidelines for pediatric resident education.

The survey you are about to participate in will explore your perceptions regarding communication disorders and AAC as part of your training. There are no known risks for your participation in this research study. The information collected may not benefit you directly. The information learned in this study may be helpful to others. The knowledge gained from your participation could help our University's medical program in its provision of a quality education and national organizations, such as the ACGME, in their provision of continuing education and their understanding of current areas of need. Your transcribed responses will be stored at the researcher's locked file cabinet in her office. The survey will last about fifteen minutes. 
The study you are about to participate in deals with your perceptions regarding communication disorders and AAC as part of your training. You will be asked to respond to these statements on a scale of 1-5, 1 being strongly disagree and 5 being strongly agree. When making your choice, do not be influenced by previous choices. It is important that you respond your actual beliefs and not according to how you feel you should believe.

Upon completion of the survey, your name will be added to a list of all participants which will be kept separate from the survey responses. One name will be randomly drawn from this list and that participant will be awarded a $\$ 200.00$ gift basket. The gift basket will be received at Weisskopf Child Evaluation Center.

Individuals from the Department of Teaching and Learning, College of Education and Human Development, the Institutional Review Board (IRB), the Human Subjects Protection Program Office (HSPPO), and other regulatory agencies may inspect these records. In all other respects, however, the data will be held in confidence to the extent permitted by law. Should the data be published, your identity will not be disclosed.

Taking part in this study is voluntary. By signing this document you agree to take part in this research study. You do not have to answer any questions that make you uncomfortable. You may choose not to take part at all. If you decide to be in this study you may stop taking part at any time. If you decide not to be in this study or if you stop taking part at any time, you will not lose any benefits for which you may qualify.

If you have any questions, concerns, or complaints about the research study, please contact: Debra Bauder, 502-852-0564.

If you have any questions about your rights as a research subject, you may call the Human Subjects Protection Program Office at (502) 852-5188. You can discuss any questions about your rights as a research subject, in private, with a member of the Institutional Review Board (IRB). You may also call this number if you have other questions about the research, and you cannot reach the study doctor, or want to talk to someone else. The IRB is an independent committee made up of people from the University community, staff of the institutions, as well as people from the community not connected with these institutions. The IRB has reviewed this research study.

If you have concerns or complaints about the research or research staff and you do not wish to give your name, you may call 1-877-852-1167. This is a 24 hour … hot line answered by people who do not work at the University of Louisville. 
Sincerely,

Debra K. Bauder, Ed.D.

Associate Professor

Karen Coulter, M.S., C.C.C./S.L.P., A.T.P.

Rm. 156, College of Education and Human Development

University of Louisville

Doctoral Candidate

Department of Pediatrics

Weisskopf Child Evaluation Center

Louisville, KY 40292

571 South Floyd Street

Louisville, KY 40202 


\begin{abstract}
Appendix E
Survey of Pediatric Residents:

Communication Disorders and

Augmentative and Alternative Communication (AAC)

Final Expert Panel Copy
\end{abstract}




\section{Survey of Pediatric Residents: \\ Communication Disorders and \\ Augmentative and Alternative Communication (AAC)}

The following questions address your perceptions about your ability to provide augmentative and alternative communication (AAC) services.

DIRECTIONS: For each statement listed below, circle the ONE number that represents your level of agreement. Use the following scale:

$$
\begin{aligned}
& 5=\text { (SA) STRONGLY AGREE } \\
& 4=\text { (A) AGREE } \\
& 3=\text { (N) NEITHER AGREE NOR DISAGREE } \\
& 2=\text { (D) DISAGREE } \\
& 1=\text { (SD) STRONGLY DISAGREE }
\end{aligned}
$$

\begin{tabular}{|c|c|c|c|c|c|c|c|}
\hline \multirow[b]{2}{*}{ ACEIEE } & \multicolumn{2}{|r|}{ ST AIEMENT } & \multicolumn{5}{|c|}{ LEVEL OF AGREEUENT } \\
\hline & & & SA & A & M & D & SD \\
\hline 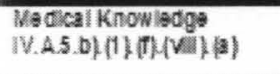 & 1. & $\begin{array}{l}\text { I participated in a blook rotation for exper ience in } \\
\text { behavioral developmental pediatrics. }\end{array}$ & 5 & $\odot$ & 3 & a & 0 \\
\hline 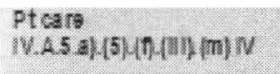 & 2. & $\begin{array}{l}\text { I have had practiced-based learning regarding } \\
\text { communication discroers. }\end{array}$ & 5 & (c) & 3 & (8) & c \\
\hline 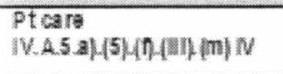 & 3. & $\begin{array}{l}\text { I have had practiced-bas ed learning regarding } \\
\text { augmentative and alter native communication dis orders }\end{array}$ & 5 & 9 & 3 & (c) & 0 \\
\hline 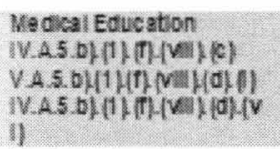 & 4. & $\begin{array}{l}\text { I have had educational training for the management of a } \\
\text { child with a communication dis order. }\end{array}$ & 5 & (2) & a & 3 & c \\
\hline 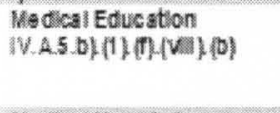 & 5. & $\begin{array}{l}\text { I have had educational training for the management of a } \\
\text { child with an augmentative and alternative } \\
\text { communication system. }\end{array}$ & 5 & Q & 3 & (3) & ○ \\
\hline 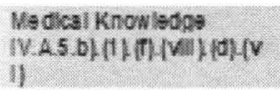 & 6. & I can recognize abnormal communication development. & 5 & 6 & 3 & a & c) \\
\hline 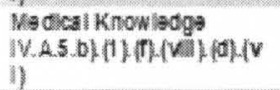 & 7. & I can recognize of abnormal speech development. & 5 & (อ) & 0 & (2) & (0) \\
\hline 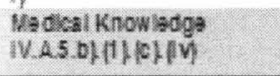 & 8. & $\begin{array}{l}\text { I have knowledge of communication disorders as a care } \\
\text { coor dingtor. }\end{array}$ & 5 & (c) & 3 & (3) & c) \\
\hline 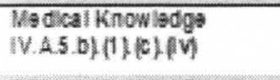 & 9. & $\begin{array}{l}\text { I have knowledge of gugmentative and alternative } \\
\text { communication devices as a care coordinator. }\end{array}$ & 5 & 0 & 3 & $a$ & 9 \\
\hline 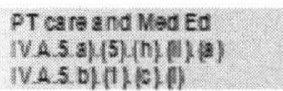 & 10. & $\begin{array}{l}\text { I unders tand the impact of a commurication discrder on } \\
\text { a child's develooment pattern and educational success. }\end{array}$ & 5 & (c) & $a$ & (2) & (1) \\
\hline 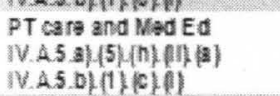 & 11. & $\begin{array}{l}\text { I understand the impact of an AAC sys tem on a child's } \\
\text { developmental pattern and educatioral success. }\end{array}$ & 5 & 0 & 3 & (3) & 0 \\
\hline $\begin{array}{l}\text { Systems besad practos } \\
\text { W.A.S.f }\{4\}\end{array}$ & 12. & $\begin{array}{l}\text { I sovocate for children to receive quality patient } \\
\text { intervention sevices for communication disor ders. }\end{array}$ & 5 & (อ) & (3) & (a) & (c) \\
\hline 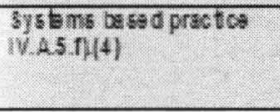 & 13. & $\begin{array}{l}\text { I acvocate for children to receive quality patient } \\
\text { intervention sevices for augmentative and alternative } \\
\text { communication systems. }\end{array}$ & 5 & 0 & (3) & e & (9) \\
\hline
\end{tabular}

In responding to the statement, think about your current work position(s) and your role regarding communication disorders and augmentative and alternative communication with patients or potential patients.

\section{Survey questions:}




\section{Survey of Pediatric Residents: Communication Disorders and Augmentative and Alternative Communication (AAC) Continued}

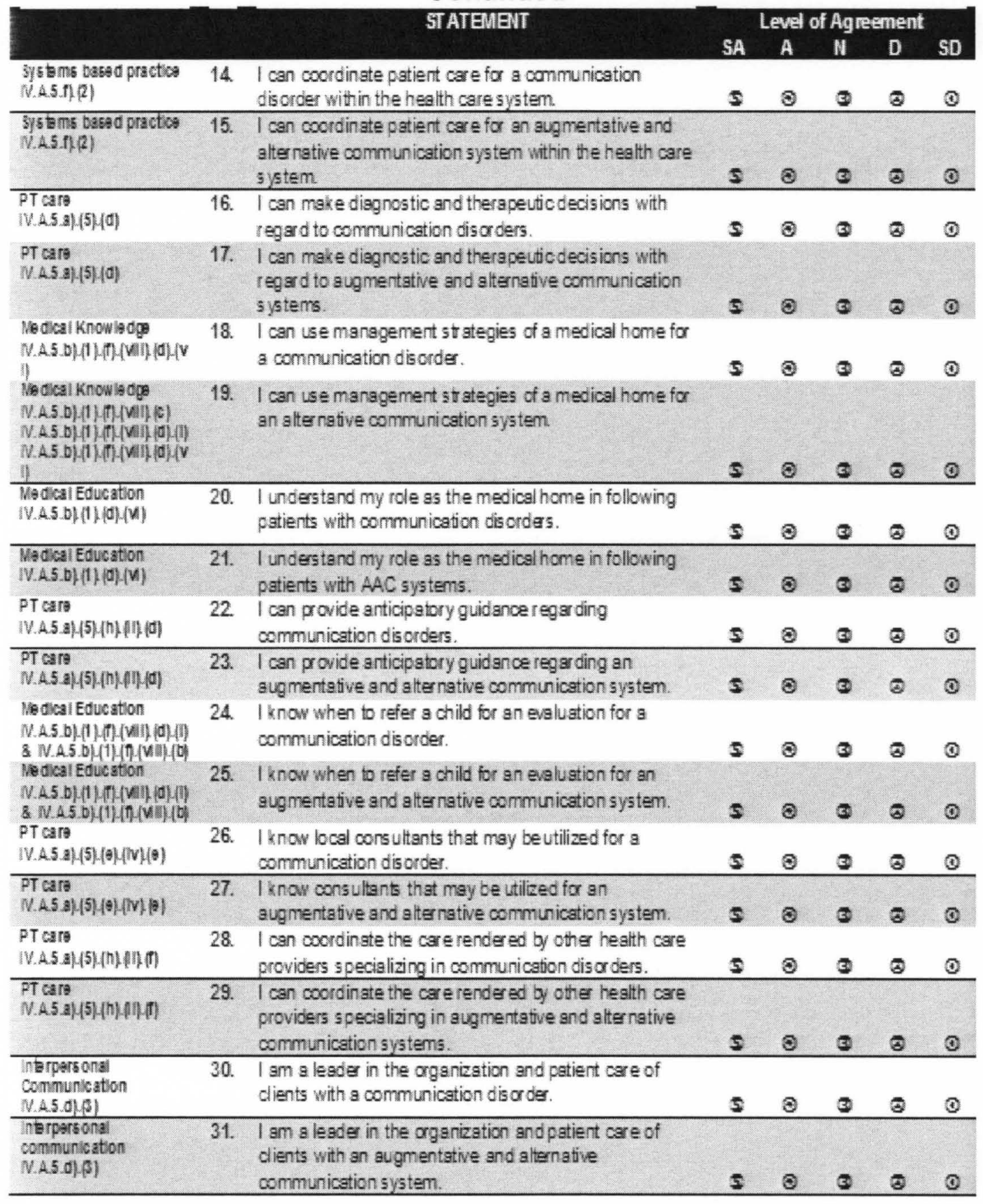




\section{Survey of Pediatric Residents: \\ Communication Disorders and Augmentative and Alternative Communication (AAC) continued}

Directions: Please mark the appropriate response in the space provided next to the statement.

1. What Pediatric Level (PL) are you currently?

$$
\begin{aligned}
& 1(P L-1) \\
& 2(P L-2) \\
& 3(P L-3)
\end{aligned}
$$

2. What rotations have you completed?

Adolescent medicine...

Newborn.

Hematology/oncology.

Emergency medicine.

Ambulatory pediatrics services....

Allergy/immunology.

Cardiology

Child development

Community health programs

Critical Care Medicine.

Dermatology

Endocrinology

Forensics.

Gastroenterology.

Genetics.
(Mark all that apply)

In-patient service (Wards).

Infectious diseases.

Neonatology....

Nephrology

Neurology

Pathology

Pediatric ICU.

Primary care

Pulmonology

Radiology.

Rheumatology

Special surgical clinics

Private practitioner's office.

Research activity.

WCEC.

(Mark one)

3. Do you plan to pursue any specialization? Yes $\square$ No

\section{What specialization(s) do you plan to pursue?}

Adolescent Medicine

Allergy and Immunology

Ambulatory Pediatrics

Cardiology

Critical Care Medicine

Developmental/ Behavioral

Pediatrics

Emergency Medicine

Endocrinology

Forensic Medicine

Gastroenterology

General Inpatient Medicine

(Hospitalists)

General Pediatrics (community

practice)
(Mark those that apply)

$\square$ Medical Genetics

Hematology/Oncology and Bone Marrow Transplant

$\square \quad$ Infectious Diseases

$\square$ International Pediatrics

$\square$ Medical History, Ethics and Humanities

$\square$ Neonatal Medicine

Nephrology and Hypertension

Pulmonary Medicine

Radiology

Rheumatology

Sleep Medicine

Other 


\section{Survey of Pediatric Residents: \\ Communication Disorders and \\ Augmentative and Alternative Communication (AAC) \\ continued}

5. What is your gender?

Male.

(Mark one)

Female.

6. Have you completed rotations where communication disorders were discussed?

Yes.

(Mark one)

No.

$\square$

* If 'No' skip to \# 9.

7. If yes then mark the format of presentation:

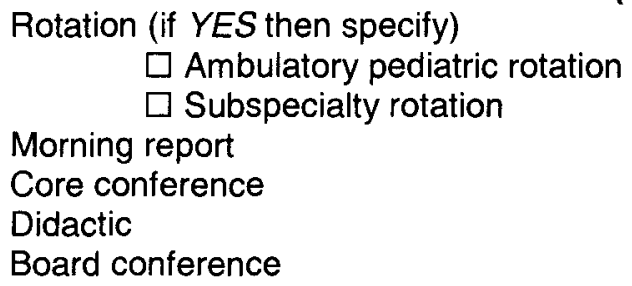

8. On average how many hours were communication disorders discussed?

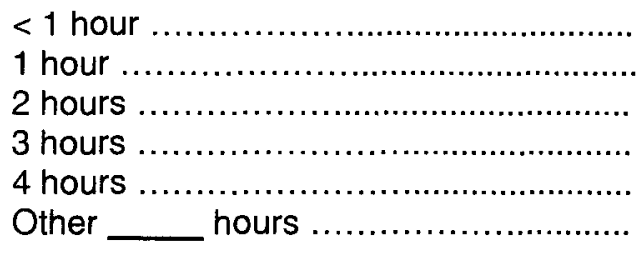

(Mark one)

9. Have you completed rotations where AAC were discussed?

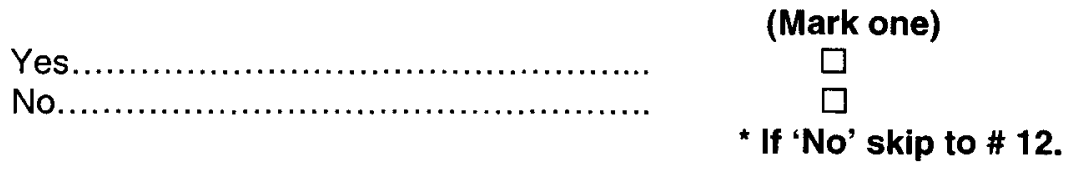

10. If yes then mark the format of presentation:

Rotation (if YES then specify)

(Mark those that apply)

$\square$ Ambulatory pediatric rotation

$\square$ Subspecialty rotation

Morning report

Core conference

Didactic

Board conference 


\section{Survey of Pediatric Residents: \\ Communication Disorders and \\ Augmentative and Alternative Communication (AAC) continued}

11. On average how many hours were AAC discussed?

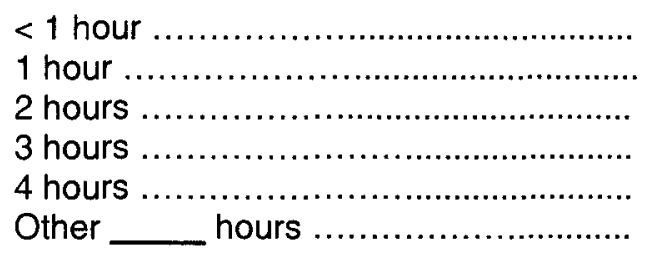

(Mark one)

$\square$

$\square$

$\square$

$\square$

12. On average how many patients do you see within your continuity clinic on any particular day?

13. On average how many patients have a diagnosed communication disorder?

14. On average how many patients use AAC?

15. How many patients could potentially use AAC? 


\begin{abstract}
Appendix F
Survey of Pediatric Residents: Communication Disorders and Augmentative and Alternative Communication (AAC): Survey Monkey Copy
\end{abstract}




\section{Survey of Pediatric Residents: Communication Disorders and Augmentative and Alternative Communication (AAC): Survey Monkey Copy}

\section{Survey of Pediatric Residents: Communication Disorders and AAC}

Pediatric Residents: Communication Disorders and

Augmentative and Attemative Communication (AAC)

Dear Pediatric Resident:

You are being invited to participate in a research study by participating in a survey to examine your perceptions of your competence in relation to Communication Disorders and AAC.

Pediatricians. the American Academy of Pediatrics and the federal government believe that pediatricians should have a significant role in the provision and supervision of therapies and Augmentative and Alternative Communication (AAC) (Sneed, et al. 2004). Within their practice, pediatricians are to act as a medical home for children and take on the ethical responsibility to coordinate the care of a child up to age twenty-one (Brewer, McPherson, Magrab, \& Hutchins, 1989; American Academy of Pediatrics, 2005:American Academy of Pediatrics, 1999). Pediatricians must have knowledge of many aspects of a child's development or lack the-eof, including a potential need for speech-language therapy and augmentative and alternative communication (AAC).

- Communication Disorders are impairments in an individual's "ability to receive, send, process, and comprehend concepts or verbal, nonverbal and graphic symbol systems" (American Speech-Language-Hearing Association, 1993). - Augmentative and alternative communication (AAC) is an area of clinical and educational practice that offers a set of strategies and approaches to supplement or replace natural speech and/or handwriting.

- Competence within this survey is framed by the ACGME guidelines for pediatric resident education.

The survey you are about to participate in will explore your perceptions regarding communication disorders and AAC as part of your training. There are no known risks for your participation in this research study. The information collected may not benefit you directly. The information learned in this study may be helpful to others. The knowledge gained from your participation could help our University's medical program in its provision of a quality education and national organizations. such as the ACGME, in their provision of continuing education and their understanding of current areas of need. Your transcribed responses will be stored at the researcher's locked file cabinet in her office. The survey will last about fifteen minutes.

The study you are about to participate in deals with your perceptions regarding communication disordeis and AAC as part of your training. You will be asked to respond to these statements on a scale of $1-5,1$ being strongly disagree and 5 being strongly agree. When making your choice, do not be influenced by previous choices. It is important that you respond your actual beliefs and not according to how you feel you should believe.

Individuals from the Department of Teaching and Leaming, College of Education and Human Development, the Institutional Review Board (IRB), the Human Subjects Protection Program Office (HSPPO), and other regulatory agencies may inspect these records. In all other respects, however, the data will be held in confidence to the extent permitted by law. Should the data be published, your identity will not be disclosed.

Taking part in this study is voluntary. By signing this document you agree to take part in this research study. You do not have to answer any questions that make you uncomfortable. You may choose not to take part at all. If you decide to be in this study you may stop taking part at any time. If you decide not to be in this study or if you stop taking part at any time, you will not lose any benefits for which you may qualify.

If you have any questions, concerns. or complaints about the research study, please contact: Debra Bauder, 502-8520564 .

If you have any questions about your rights as a research subject, you may call the Human Subjects Protection Program Office at (502) 852-5188. You can discuss any questions about your rights as a research subject, in private, with a member of the Institutional Review Board (IRB). You may also call this number if you have other questions about the research, and you cannot reach the study doctor, or want to talk to someone else. The IRB is an independent committee made up of people from the University community, staff of the institutions, as well as people from the community not connected with these institutions. The IRB has reviewed this research study. 
Survey of Pediatric Residents: Communication Disorders and AAC

call 1-877-852-1167. This is a 24 hour hot line answered by people who do not work at the University of Louisville.

Sincerely,

Debra K. Bauder, Ed.D.

Associate Professor

Rm. 156, College of Education and Human Development

University of Loulsville Louisville. KY 40292

Karen Coulter, M.S. C.C.C.IS.L.P., A.T.P.

Doctoral Candidate

Department of Pediatrics

Weisskopf Child Evaluation Center

571 South Floyd Street

Louisville, KY 40202 


\section{Survey of Pediatric Residents: Communication Disorders and AAC}

*1. The following questions address your perceptions about your ability to provide augmentative and alternative communication (AAC) services.

DIRECTIONS: For each statement listed below, circle the ONE number that represents your level of agreement. Use the following scale:

$5=(\mathrm{SA})$ STRONGLY AGREE

$4=($ A) AGREE

$3=(\mathrm{N})$ NEITHER AGREE NOR DISAGREE

2 = (D) DISAGREE

$1=(\mathrm{SD})$ STRONGLY DISAGREE

In responding to the statement, think about your current work position(s) and your role regarding communication disorders and augmentative and alternative communication with patients or potential patients.

Survey questions:

I participated in a block

behavioral/ developmental

pediatrics.

I have had practiced-based

learning regarding

communication disorders.

I have had practiced-based

learning regarding

augmentative and

alternative communication

disorders

I have had educational

training for the

management of a child

with a communication

disorder.

I have had educational

training for the

management of a child

with an augmentative and

alternative communication

system.

I can recognize abnorma|

communication

development.

I can recognize of

abnormal speech

development.

I have knowledge of

communication disorders a

a care coordinator.

$\stackrel{S D}{O}$

0

0

0

0

$\mathrm{N}^{N}$

$\stackrel{A}{O}$

0

0

0

0

0

0

0

0

0

0

0

0

0

0

0

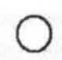

0

0

0

0

0<smiles>[CH]C</smiles><smiles>[GeH2]</smiles>

0

0

0<smiles>[CH]</smiles>

0<smiles>C1CCC1</smiles>

0

0

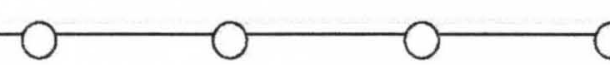




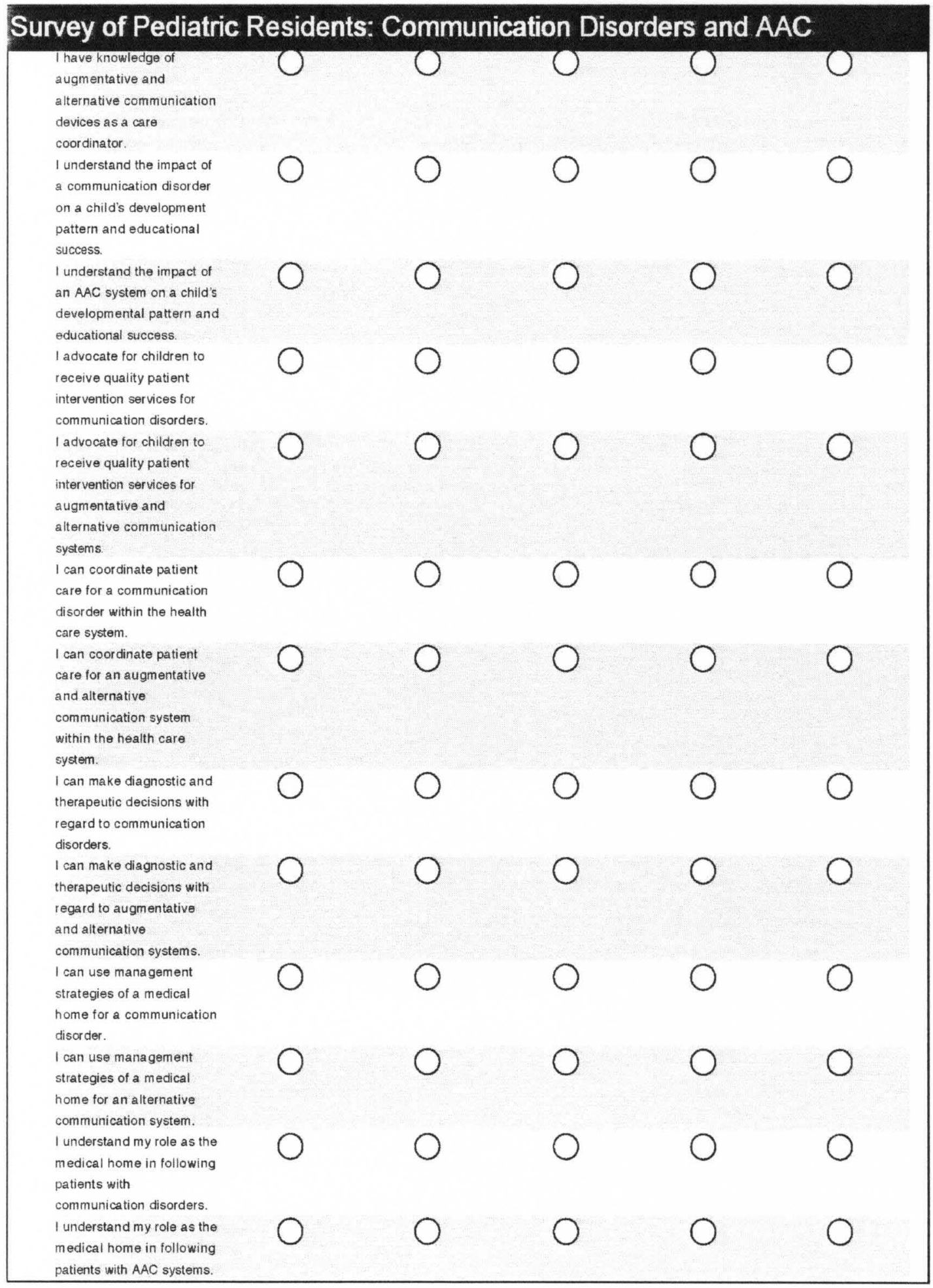




\section{Survey of Pediatric Residents: Communication Disorders and AAC}

I can provide anticipatory

guidance regarding

communication disorders.

I can provide anticipatory

guidance regarding an

augmentative and

alternative communication

system

I know when to refer a child

for an evaluation for a

communication disorder.

I know when to refer a child

for an evaluation for an

augmentative and

alternative communication

system.

I know local consultants

that may be utilized for a

communication disorder.

I know consultants that may

be utilized for an

augmentative and

alternative communication

system.

I can coordinate the care

rendered by other health

care providers specializing

in communication

disorders.

I can coordinate the care

rendered by other health

care providers specializing

in augmentative and

alternative communication

systems.

I am a leader in the

organization and patient

care of clients with a

communication disorder.

I am a leader in the

organization and patient

care of clients with an

augmentative and

alternative communication

system.
$\mathrm{O}$

$\mathrm{O}$

O

O

O

0

○

O

O

O

0

O

0

0

0

O

O

○

O

$\bigcirc$

O

O

O

○

O

O

O

O

O

$\bigcirc$

O 0

O

O

O

O

O

O

O

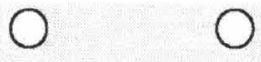

O

* 2. What is your gender?

male

Female

* 3. Do you plan to pursue any specialization?

$\bigcirc$ Yes

№ 


\section{Survey of Pediatric Residents: Communication Disorders and AAC}

4. What specialization(s) do you plan to pursue?

Select your specialization:

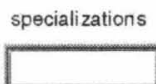

* 5. What Pediatric Level (PL) are you currently?
$\mathrm{O}_{1(\mathrm{PL}-1)}$
$\mathrm{2}_{2(\mathrm{PL}-2)}$
$\mathrm{O}_{3(\mathrm{PL}-3)}$

* 6. What rotations have you completed?

$\square$ Adolescent medicine
$\square$ Ambulatory pediatrics services
$\square$ Allergy fimmunology
$\square$ Cardiology
$\square$ Child development
$\square$ Community health programs
$\square$ Critical Care Medicine
$\square$ Dermatology
$\square$ Endocrinology
$\square$ Emergency medicine

$\square$ Forensics
$\square$ Gastroenterology
$\square$ Genetics
$\square$ Hematologyloncology
$\square$ In-patient service (Wards
$\square$ Infectious diseases
$\square$ Neonatology
$\square$ Nephrology
$\square$ Neurology
$\square$ Newborn

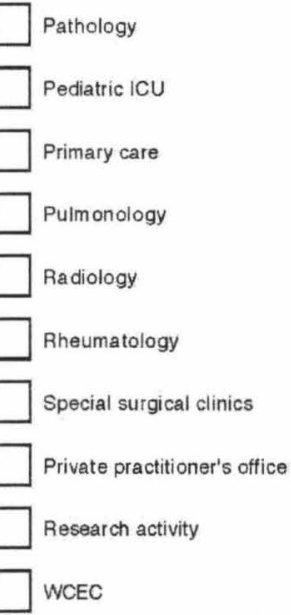

* 7. Have you completed rotations where communication disorders were discussed? * If 'No' skip to \#10.

Yes No

8. If yes then mark the format of presentation:

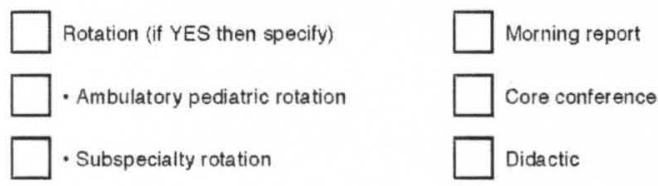

9. On average how many hours were communication disorders discussed?
$\bigcirc<1$ hour
O hour
2hours
3 hours
4hours
other hours

* 10. Have you completed rotations where AAC was discussed?

*If 'No' skip to \# 13.

Yes

No 


\section{Survey of Pediatric Residents: Communication Disorders and AAC}

11. If yes then mark the format of presentation:

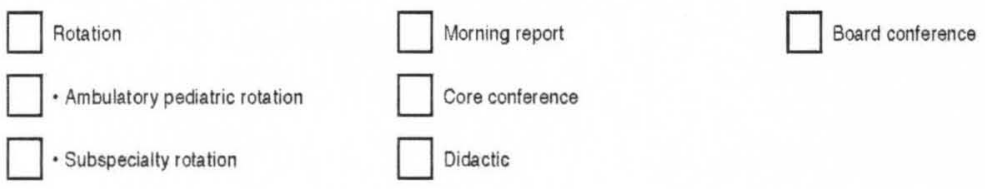

12. On average how many hours were AAC discussed?

O $<1$ hour

Onour

2hours

3 hours

Ohours

other

* 13. On average how many patients do you see within your continuity clinic on any particular day?

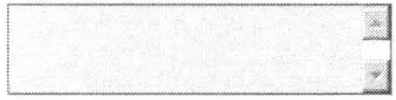

* 14. On average how many patients have a diagnosed communication disorder?

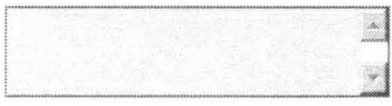

* 15. On average how many patients use AAC?

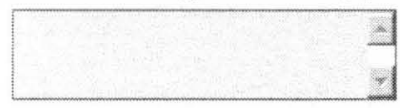


Appendix G

Focus Group

Participation Consent 


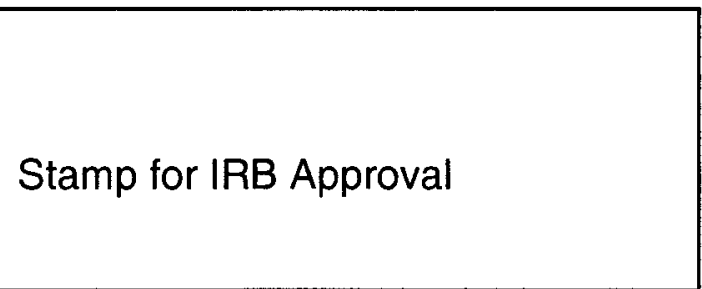

Focus Group for Pediatric Residents: Communication Disorders and Augmentative and Alternative Communication (AAC)

Date:

Dear Pediatric Resident:

You are being invited to participate in a research study by participating in a focus group to examine your perceptions of your competence in relation to Communication Disorders and AAC.

Pediatricians, the American Academy of Pediatrics and the federal government believe that pediatricians should have a significant role in the provision and supervision of therapies and Augmentative and Alternative Communication (AAC) (Sneed, et al. 2004). Within their practice, pediatricians are to act as a medical home for children and take on the ethical responsibility to coordinate the care of a child up to age twenty-one (Brewer, McPherson, Magrab, \& Hutchins, 1989; American Academy of Pediatrics, 2005;American Academy of Pediatrics, 1999). Pediatricians must have knowledge of many aspects of a child's development or lack thereof, including a potential need for speech-language therapy and augmentative and alternative communication (AAC).

- Communication Disorders are impairments in an individual's "ability to receive, send, process, and comprehend concepts or verbal, nonverbal and graphic symbol systems" (American Speech-Language-Hearing Association, 1993).

- Augmentative and alternative communication (AAC) is an area of clinical and educational practice that offers a set of strategies and approaches to supplement or replace natural speech and/or handwriting.

- Competence within this survey is framed by the ACGME guidelines for pediatric resident education.

This focus group you are about to participate in will explore your perceptions regarding communication disorders and AAC as part of your training. There are no known risks for your participation in this research study. The 
information collected may not benefit you directly. The information learned in this study may be helpful to others. The knowledge gained from your participation could help our University's medical program in its provision of a quality education and national organizations, such as the ACGME, in their provision of continuing education and their understanding of current areas of need. Your transcribed responses will be stored at the researcher's locked file cabinet in her office. The focus group will last about thirty minutes.

You will be asked to respond to several opened ended questions to begin the discussion. When you respond, do not be influenced by other's statements. It is important that you respond your actual beliefs and not according to how you feel you should believe.

Individuals from the Department of Teaching and Learning, College of Education and Human Development, the Institutional Review Board (IRB), the Human Subjects Protection Program Office (HSPPO), and other regulatory agencies may inspect these records. In all other respects, however, the data will be held in confitent permitted by law. Should the data be published, your identity will not be disclosed.

Taking part in this study is voluntary. By signing this document you agree to take part in this research study. You do not have to answer any questions that make you uncomfortable. You may choose not to take part at all. If you decide to be in this study you may stop taking part at any time. If you decide not to be in this study or if you stop taking part at any time, you will not lose any benefits for which you may qualify.

If you have any questions, concerns, or complaints about the research study, please contact: Debra Bauder, 502-852-0564. If you have any questions about your rights as a research subject, you may call the Human Subjects Protection Program Office at (502) 852-5188. You can discuss any questions about your rights as a research subject, in private, with a member of the Institutional Review Board (IRB). You may also call this number if you have other questions about the research, and you cannot reach the study doctor, or want to talk to someone else. The IRB is an independent committee made up of people from the University community, staff of the institutions, as well as people from the community not connected with these institutions. The IRB has reviewed this research study.

If you have concerns or complaints about the research or research staff and you do not wish to give your name, you may call 1-877-852-1167. This is a 24 hour hot line answered by people who do not work at the University of Louisville. 
Sincerely,

Debra K. Bauder, Ed.D.

Karen Coulter, M.S., C.C.C./S.L.P., A.T.P. Associate Professor

Rm. 156, College of Education and Doctoral Candidate

Human Development

University of Louisville

Department of Pediatrics

Weisskopf Child Evaluation Center

571 South Floyd Street

Louisville, KY 40292

Louisville, KY 40202

Participant Signature

Principal Investigator

Focus Group Moderator/Student Investigator 
Appendix H

Focus Group Flier 
Focus Group Flier

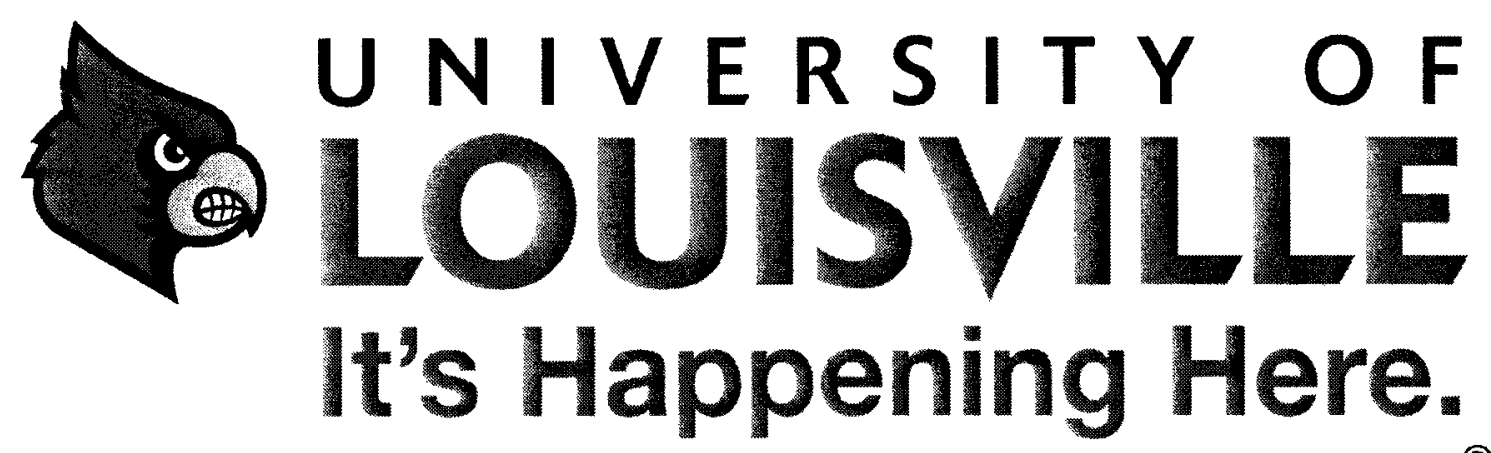

Calling All Pediatric Residents!

(B)

You are being invited to participate in a focus group to understand your perceptions of communication disorders and $A A C$ as part of your training program. The research discussion will occur during lunch. $A$ FREE LUNCH will be provided for all participants.

Date: December $13^{\text {th }}, 2010$

Time: 12 o'clock

Location: $6^{\text {th }}$ floor conference room

The knowledge gained from your responses could help our University's medical program in its provision of a quality education and national organizations, such as the ACGME, in their provision of continuing education and improve their understanding of current areas of need. For further information regarding this study contact Karen Coulter at (502)852-1420.

Thank-you for your time and support!

Debra K. Bauder, Ed.D.

Rm. 156, College of Education and Human Development University of Louisville 


\section{Appendix I}

Survey Flier 


\section{Survey Flier}

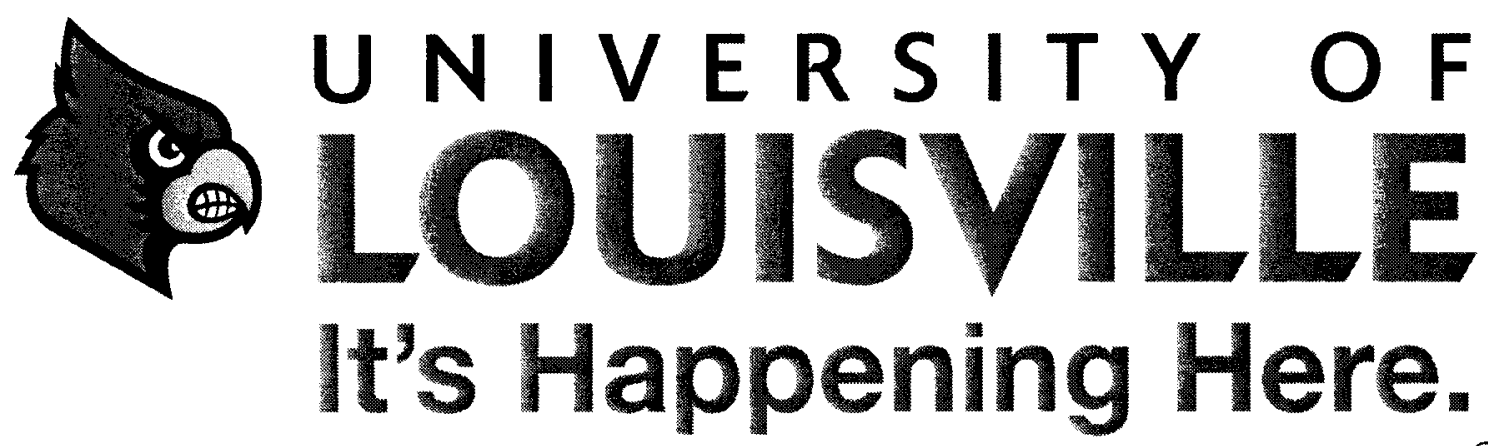

You are being invited to participate in a research study designed to measure pediatric resident's perception of their competence in relation to Communication Disorders and AAC. The survey should take about 15 minutes to complete.

- Communication Disorders are impairments in an individual's "ability to receive, send, process, and comprehend concepts or verbal, nonverbal and graphic symbol systems" (American Speech-Language-Hearing Association, 1993).

- Augmentative and alternative communication (AAC) is an area of clinical and educational practice that offers a set of strategies and approaches to supplement or replace natural speech and/or handwriting.

- Competence within this survey is framed by the ACGME guidelines for pediatric resident education.

Participation within this survey will enter you within a drawing for a two-hundred dollar gift basket. This will include various items such as a Marathon gift card, certificate for free spa services, and other goodies.

The knowledge gained from your survey responses could help our University's medical program in its provision of a quality education and national organizations, such as the ACGME, in their provision of continuing education and improve their understanding of current areas of need. For further information regarding this study contact Karen Coulter at (502)852-1420.

Thank-you for your time and support!

Debra K. Bauder, Ed.D.

Rm. 156, College of Education and Human Development University of Louisville 
Appendix J

Email Communication 


\section{Email Communication}

Thanks for your participation!

Mailed on December 21, 2010 10:13 AM $\quad 4$

LAST CHANCE for $\$ 200$ gift drawing for survey completion

Mailed on December 21,2010 10:00 AM 5

6

$\$ 200$ gift drawing for Research Survey Completion

Mailed on December 15, 2010 1:00 PM $\quad 6$

Pediatric Survey with

$\$ 200$ dollar gift card

drawing

Mailed on December 10, 2010 9:13 AM $\quad \begin{aligned} & 7 \\ & 8\end{aligned}$

Pediatric Resident

Survey - We need to Mailed on December 1, 2010 4:07 PM 1

hear from you

Pediatric Resident

Survey - We need to

hear from you

Mailed on December 1, 2010 4:01 PM $\quad 9$
6

$U$ of L Pediatric

Resident Research

Survey

Mailed on November $29,20106: 00$ AM 1

Subject: Thanks for your participation!

Body: Dear [FirstName],

Thank-you for your participation in the Pediatric Resident Research survey! You have been entered into the drawing for the $\$ 200$ gift card drawing (free massage, Marathon gas card, Kroger/ Jay-C grocery card, Target card, Calistoga card, and Best Buy card).

The winner of the drawing will be announced at the end of the survey period, December 28th. That lucky individual will be contacted on the 29th via email, and the prize will be made 
available.

Thanks again for your help! If you have any further questions, feel free to contact me at (502)852-1420.

Sincerely,

Karen Coulter

http://www.surveymonkey.com/optout.aspx

http://www.surveymonkey.com/s.aspx

\section{Subject:}

LAST CHANCE for $\$ 200$ gift drawing for survey completion

\section{Body:}

The deadline for survey completion and your chance to participate in the $\$ 200$ drawing is December 28th. We would like to hear from you!

You are being invited to participate in a research study designed to measure pediatric resident's perception of their competence in relation to Communication Disorders and AAC. The survey should take about 15 minutes to complete.

Here is a link to the survey:

http://www.surveymonkey.com/s.aspx

This link is uniquely tied to this survey and your email address. Please do not forward this message.

-Communication Disorders are impairments in an individual's "ability to receive, send, process, and comprehend concepts or verbal, nonverbal and graphic symbol systems" (American Speech-Language-Hearing Association, 1993).

-Augmentative and alternative communication (AAC) is an area of clinical and educational practice that offers a set of strategies and approaches to supplement or replace natural speech and/or handwriting.

-Competence within this survey is framed by the ACGME guidelines for 
pediatric resident education.

Participation within this survey will enter you within a drawing for a twohundred dollar gift basket. This will include various items such as a Marathon gift card, certificate for free spa services, and other goodies.

The knowledge gained from your survey responses could help our University's medical program in its provision of a quality education and national organizations, such as the ACGME, in their provision of continuing education and improve their understanding of current areas of need.

Thank-you!

Please note: If you do not wish to receive further emails from us, please click the link below, and you will be automatically removed from our mailing list.

http://www.surveymonkey.com/optout.aspx

Subject: $\$ 200$ gift drawing for Research Survey Completion

Body: We haven't heard from you yet!

You are being invited to participate in a research study designed to measure pediatric resident's perception of their competence in relation to Communication Disorders and AAC. The survey should take about 15 minutes to complete.

Here is a link to the survey:

http://www.surveymonkey.com/s.aspx

This link is uniquely tied to this survey and your email address. Please do not forward this message.

-Communication Disorders are impairments in an individual's "ability to receive, send, process, and comprehend concepts or verbal, nonverbal and graphic symbol systems" (American Speech-Language-Hearing Association, 1993).

-Augmentative and alternative communication (AAC) is an area of clinical and educational practice that offers a set of strategies and approaches to supplement or replace natural speech and/or handwriting. 
- Competence within this survey is framed by the ACGME guidelines for pediatric resident education.

Participation within this survey will enter you within a drawing for a two-hundred dollar gift basket. This will include various items such as a Marathon gift card, certificate for free spa services, and other goodies.

The knowledge gained from your survey responses could help our University's medical program in its provision of a quality education and national organizations, such as the ACGME, in their provision of continuing education and improve their understanding of current areas of need.

Thank-you!

Please note: If you do not wish to receive further emails from us, please click the link below, and you will be automatically removed from our mailing list.

http://www.surveymonkey.com/optout.aspx

\section{Subject:}

Pediatric Survey with $\$ 200$ dollar gift card drawing

\section{Body:}

We haven't heard from you yet!

You are being invited to participate in a research study designed to measure pediatric resident's perception of their competence in relation to Communication Disorders and AAC. The survey should take about 15 minutes to complete.

Here is a link to the survey:

http://www.surveymonkey.com/s.aspx

This link is uniquely tied to this survey and your email address. Please do not forward this message.

-Communication Disorders are impairments in an individual's "ability to receive, send, process, and comprehend concepts or verbal, nonverbal and graphic symbol systems" (American Speech-Language-Hearing Association, 1993). 
-Augmentative and alternative communication (AAC) is an area of clinical and educational practice that offers a set of strategies and approaches to supplement or replace natural speech and/or handwriting.

-Competence within this survey is framed by the ACGME guidelines for pediatric resident education.

Participation within this survey will enter you within a drawing for a twohundred dollar gift basket. This will include various items such as a Marathon gift card, certificate for free spa services, and other goodies.

The knowledge gained from your survey responses could help our University's medical program in its provision of a quality education and national organizations, such as the ACGME, in their provision of continuing education and improve their understanding of current areas of need.

Thank-you!

Please note: If you do not wish to receive further emails from us, please click the link below, and you will be automatically removed from our mailing list. http://www.surveymonkey.com/optout.aspx

Pediatric Resident Survey - We need to hear from you

Body: We need your input!

You are being invited to participate in a research study designed to measure pediatric resident's perception of their competence in relation to Communication Disorders and AAC. The survey should take about 15 minutes to complete.

Here is a link to the survey:

http://www.surveymonkey.com/s.aspx

This link is uniquely tied to this survey and your email address.

Please do not forward this message.

-Communication Disorders are impairments in an individual's "ability to receive, send, process, and comprehend concepts or verbal, nonverbal and graphic symbol systems" (American Speech- 
Language-Hearing Association, 1993).

-Augmentative and alternative communication (AAC) is an area of clinical and educational practice that offers a set of strategies and approaches to supplement or replace natural speech and/or handwriting.

-Competence within this survey is framed by the ACGME guidelines for pediatric resident education.

Participation within this survey will enter you within a drawing for a two-hundred dollar gift basket. This will include various items such as a Marathon gift card, certificate for free spa services, and other goodies.

The knowledge gained from your survey responses could help our University's medical program in its provision of a quality education and national organizations, such as the ACGME, in their provision of continuing education and improve their understanding of current areas of need.

Thank-you!

Please note: If you do not wish to receive further emails from us, please click the link below, and you will be automatically removed from our mailing list.

Subject: Pediatric Resident Survey - We need to hear from you

Body: We haven't heard from you yet!

You are being invited to participate in a research study designed to measure pediatric resident's perception of their competence in relation to Communication Disorders and AAC. The survey should take about 15 minutes to complete.

Here is a link to the survey:

http://www.surveymonkey.com/s.aspx

This link is uniquely tied to this survey and your email address. Please do not forward this message. 
-Communication Disorders are impairments in an individual's "ability to receive, send, process, and comprehend concepts or verbal, nonverbal and graphic symbol systems" (American Speech-Language-Hearing Association, 1993).

-Augmentative and alternative communication (AAC) is an area of clinical and educational practice that offers a set of strategies and approaches to supplement or replace natural speech and/or handwriting.

-Competence within this survey is framed by the ACGME guidelines for pediatric resident education.

Participation within this survey will enter you within a drawing for a two-hundred dollar gift basket. This will include various items such as a Marathon gift card, certificate for free spa services, and other goodies.

The knowledge gained from your survey responses could help our University's medical program in its provision of a quality education and national organizations, such as the ACGME, in their provision of continuing education and improve their understanding of current areas of need.

Thank-you!

Please note: If you do not wish to receive further emails from us, please click the link below, and you will be automatically removed from our mailing list.

http://www.surveymonkey.com/optout.aspx

Subject: $U$ of L Pediatric Resident Research Survey

Body: You are being invited to participate in a research study designed to measure pediatric resident's perception of their competence in relation to Communication Disorders and AAC. The survey should take about 15 minutes to complete.

Here is a link to the survey:

http://www.surveymonkey.com/s.aspx

This link is uniquely tied to this survey and your email address. Please do not forward this message. 
-Communication Disorders are impairments in an individual's "ability to receive, send, process, and comprehend concepts or verbal, nonverbal and graphic symbol systems" (American Speech-Language-Hearing Association, 1993).

-Augmentative and alternative communication (AAC) is an area of clinical and educational practice that offers a set of strategies and approaches to supplement or replace natural speech and/or handwriting.

- Competence within this survey is framed by the ACGME guidelines for pediatric resident education.

Participation within this survey will enter you within a drawing for a two-hundred dollar gift basket. This will include various items such as a Marathon gift card, certificate for free spa services, and other goodies.

The knowledge gained from your survey responses could help our University's medical program in its provision of a quality education and national organizations, such as the ACGME, in their provision of continuing education and improve their understanding of current areas of need.

Thank-you!

Please note: If you do not wish to receive further emails from us, please click the link below, and you will be automatically removed from our mailing list.

http://www.surveymonkey.com/optout.aspx 


\section{CURRICULUM VITA}

KAREN J. COULTER

EDUCATION

Department of Education, Doctoral Candidate, Present University of Louisville, Louisville, Kentucky.

Speech Language Pathology, M.S. August 1998

Indiana State University, Terre Haute, Indiana.

Speech Language Pathology, B.S. August 1992

Illinois State University, Normal, Illinois.

LICENSURE

- ASHA Certificate of Clinical Competency

- Kentucky Professional Licensure

-RESNA Assistive Technology Practitioner

-Kentucky First Steps Provider

\section{EXPERIENCE}

Speech Language Pathologist, M.S., C.C.C./S.L.P., A.T.P. Weisskopf Child Evaluation Center

November 2004 - Present

Speech Language Pathologist, M.S., C.C.C./S.L.P. Bridgepointe Center

Summer 2001 - November 2004

Speech Language Pathologist, M.S., C.C.C./S.L.P. New Albany-Floyd County Consolidated School Corporation Fall $1998-2001$

\section{MEMBERSHIPS}

American Speech-Language-Hearing Association, Kentucky Speech-Language-Hearing Association, Inc.

RESNA

ISAAC

ASHA Committee 12: AAC 
ASHA Committee 11: Higher Education

TEACHING

University of Louisville

646 Augmentative and Alternative Communication

Spring 2009 - Fall 2009

University of Louisville

660 Assistive Technology: Introduction and Software for

Young Children

Fall 2005

GUEST LECTURER

University of Louisville

218 Technology for Students with Disabilities

Spring 2010

Spring 2011

CONFERENCES

Kentucky Speech Language and Hearing Association 2010 Conference

Autism Society of America

2005 Conference

CLINIC

Weisskopf Child Evaluation Center AAC Clinic

Fall 2010 - Present

GRANTS

Holloway: $\$ 3,500$

SPECIALIZED TRAINING

Hanen Certified Speech Language Pathologist

It Takes Two to Talk

More Than Words

Picture Exchange Communication System: Advanced

AAC Systems/ Devices: Various

AAC Assessment/ Management

Assistive Technology

Autism Spectrum Disorders

Beckman Protocol

Behavioral Feeding 


\title{
RESEARCH INTERESTS
}

\author{
AAC \\ Pediatric Feeding
}

INOVATIONS (copywrited):

Food Checklist( 